\title{
Tales on Tails
}

Citation for published version (APA):

Pollastri, A. (2018). Tales on Tails. [Doctoral Thesis, Maastricht University]. Datawyse / Universitaire Pers Maastricht. https://doi.org/10.26481/dis.20180830ap

\section{Document status and date:}

Published: 01/01/2018

DOI:

10.26481/dis.20180830ap

Document Version:

Publisher's PDF, also known as Version of record

\section{Please check the document version of this publication:}

- A submitted manuscript is the version of the article upon submission and before peer-review. There can be important differences between the submitted version and the official published version of record.

People interested in the research are advised to contact the author for the final version of the publication, or visit the DOI to the publisher's website.

- The final author version and the galley proof are versions of the publication after peer review.

- The final published version features the final layout of the paper including the volume, issue and page numbers.

Link to publication

\footnotetext{
General rights Owners
rights.

- You may freely distribute the URL identifying the publication in the public portal. please follow below link for the End User Agreement:

www.umlib.nl/taverne-license

Take down policy

If you believe that this document breaches copyright please contact us at:

repository@maastrichtuniversity.nl

providing details and we will investigate your claim.
}

Copyright and moral rights for the publications made accessible in the public portal are retained by the authors and/or other copyright owners and it is a condition of accessing publications that users recognise and abide by the legal requirements associated with these

- Users may download and print one copy of any publication from the public portal for the purpose of private study or research.

- You may not further distribute the material or use it for any profit-making activity or commercial gain

If the publication is distributed under the terms of Article $25 \mathrm{fa}$ of the Dutch Copyright Act, indicated by the "Taverne" license above, 


\section{Tales on Tails}

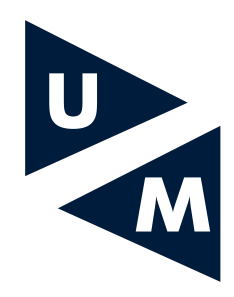

Alessandro Pollastri

Maastricht University School of Business and Economics

Maastricht, Netherlands

A thesis submitted for the degree of

Doctor of Philosophy at Maastricht University

August 30, 2018 
(c) 2018 Alessandro Pollastri

All rights reserved. No part of this publication may be reproduced, stored in a retrieval system, or transmitted, in any form, or by any means, electronic, mechanical, photocopying, recording or otherwise, without the prior permission in writing from the author.

The cover is an original work of Gabriele Tummino.

This book was typeset using $\mathrm{LT}_{\mathrm{E}} \mathrm{X}$.

ISBN 9789462959781

Printed by Datawyse / Universitaire Pers Maastricht 


\section{Tales on Tails}

\section{DISSERTATION}

to obtain the degree of Doctor at Maastricht University,

by the authority of Prof. Dr. R. Letschert, Rector Magnificus,

in accordance with the decision of the Board of Deans,

to be defended in public on

Thursday, August 30th 2018 at, 10.00

by

\section{Alessandro Pollastri}




\section{Promotors}

Prof. Dr. Peter Schotman

Dr. Stefan Straetmans

\section{Assessment committee}

Prof. Dr. Jaap Bos, Chairman

Prof. Dr. Casper De Vries

Prof. Dr. Antoon Pelsser

Prof. Dr. Roberto Renó

This research was financially supported by The Network for Studies on Pensions, Aging and Retirement (NETSPAR). 
Everything that can be counted does not necessarily count; everything that counts cannot necessarily be counted.

Albert Einstein 


\section{Acknowledgements}

Working on this dissertation has been one of the most rewarding experiences of my life, as I have enhanced my knowledge on interesting topics and also learned much about myself. This learning process led to this small book that contains hours of thoughts in front of a laptop drinking coffee. Yet, these hours of work would not have been neither as productive nor as fun if colleagues, friends and family were not there. Therefore, I will take a few lines to acknowledge those people that have been part of this fun and demanding journey.

First, I would like to express my sincere gratitude to my supervisors, Peter Schotman and Stefan Straetmans. Peter, you have always believed in me and I have learned a lot from you during our meetings. The enthusiasm you show for the topics on which we have been working on is astonishing and I have for sure benefited from it. Stefan, I have always enjoyed our dinners where we discussed many different topics, from research to arts and music. Further, you have been always there to talk to when I needed.

Next, I would like to thank the reading committee, Jaap Bos, Casper De Vries, Antoon Pelsser and Roberto Renó for reading the thesis and providing feedback to improve the manuscript. Further, the chapters in this dissertation have benefited from the many presentations at conferences and discussions with the participants.

A special thank goes to the ladies of the secretariat, Carina, Cecile, Els and Francien for providing numerous treats, such as cakes and fresh fruits, and for the support with administrative issues. Next, I would like to thank all the other numerous members of the finance department who took some time to either provide feedback on my research or to simply share a coffee.

To my paranymphs, Paulo and Matteo. Paulo, I have so many reasons to thank you. I have very much enjoyed our conversations about research and in the same manner I have appreciated our talks about football and food. ${ }^{1}$ Matteo, despite your bad taste in terms of football teams, thank you for the time spent debating Italian politics, sharing your thoughts on fashion and introducing me to modern italian musicians.

I would like to thank all my ex-officemates for the many fun times I had with them. My first officemates were Wiebke and Patrick, with the former trying to convince me to

\footnotetext{
${ }^{1}$ While I was enjoying a lobster in Lisbon, he could not stop talking about options and how great Benfica is. Later that day Benfica lost 0-3 to Sporting.
} 
learn Dutch, and the latter to play poker. ${ }^{2}$ The next officemate I had is Juan, with whom I shared pizzas from Piano B and countless nights at Peter's watching either Fiorentina or Real Madrid. Finally, I want to thank Hang and Shusen for introducing me to authentic Chinese cuisine and for teaching me very useful Chinese words.

Besides my time at the office, I enjoyed the years in Maastricht thanks to a small group of well selected friends with whom I had holidays, shared drinks and watched movies. Rogier, I want to thank you for your continuous feedback on my research, your support on various occasions and for being a valuable opponent when playing video games. Tobias, thank you for the many stimulating conversations not necessarily related to our research, and for the many entertaining moments we had. Clarissa thank you for organizing so many events at your place that helped relieve stress and distract me momentarily from my work routine. Finally, I want to thank Inka for our time together, the many discoveries we made in Maastricht, and for her ongoing friendship. ${ }^{3}$

Oltre alle persone che ho incontrato a Maastricht un grazie di cuore va a coloro che mi conoscono da sempre e durante la stesura di questa tesi sono state di supporto in qualunque modo potessero. Tra queste persone ci sono sicuramente gli amici di una vita, I ragazzi di Via Venezia, e in particolar modo Niccoló per esserci stato in momenti felici e tristi. Un ulteriore ringraziamento va a Gabriele che ha disegnato la copertina di questa tesi. Per concludere, vorrei ringraziare mia Mamma e Umberto per avermi dato ogni possibilità di arrivare fino a qui e per il loro supporto incondizionato.

\footnotetext{
${ }^{2}$ Truth be told, Wiebke managed to have me saying "Goedemorgen mooie dames" to the secretaries.

${ }^{3}$ She is the one that discovered Toscanella.
} 


\section{Contents}

Acknowledgements $\quad$ ii

List of Figures $\quad$ vi

List of Tables vii

1 Introduction 1

$\begin{array}{lll}2 & \text { A Jumping Index } & 7\end{array}$

2.1 Introduction . . . . . . . . . . . . . . . . . . 7

2.2 Model and Estimation Approach _. . . . . . . . . . . . . . . 10

2.2.1 Model . . . . . . . . . . . . . . . . . . 10

2.2.2 Estimation Approach . . . . . . . . . . . . . . . . . 12

2.3 Empirical Analysis . . . . . . . . . . . . . . . . . . . 14

2.3 .1 Data . . . . . . . . . . . . . . . . . 14

2.3.2 Parameter Estimates and Model Choice . . . . . . . . . . . . . 15

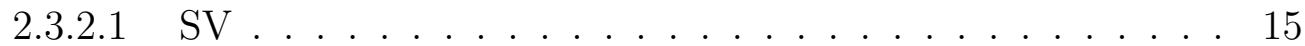

$2.3 .2 .2 \quad$ SVJ . . . . . . . . . . . . . . . 16

2.3 .2 .3 SVCJ . . . . . . . . . . . . . . . . 17

2.3.2.4 Model Choice . . . . . . . . . . . . . . . 18

2.3.3 When (and why) does the Index Jump? . . . . . . . . . . . . . 20

2.3.3.1 Simulation Results . . . . . . . . . . . . . . . 20

2.3.3.2 Jump Day Analysis . . . . . . . . . . . . . . . . . . . . 20

2.4 Conclusions . . . . . . . . . . . . . . . . . . . . . 30

3 Tailoring the Errors $\quad 35$

3.1 Introduction . . . . . . . . . . . . . . . . . . 35

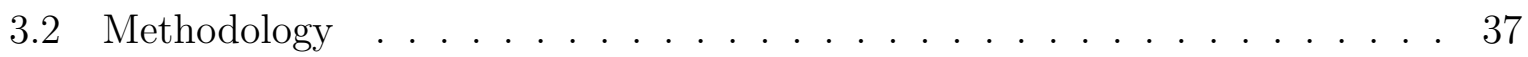

3.2 .1 Volatility Models . . . . . . . . . . . . . . . . 38

3.2.2 Diagnostic Checks . . . . . . . . . . . . . . . . 40

3.2 .3 Backtesting methods . . . . . . . . . . . . . . . 41

3.3 Results . . . . . . . . . . . . . . . . . . . . . . . . 43

3.3 .1 Data . . . . . . . . . . . . . . . 43

3.3.2 Diagnostic Checks Results . . . . . . . . . . . . . . . . . . 48

3.3 .3 VaR implications . . . . . . . . . . . . . . . . . 52

3.3.4 Backtesting Results . . . . . . . . . . . . . . . . . 55

3.3.5 Short Positions . . . . . . . . . . . . . . . . . 57 
3.3.6 Individual Stocks . . . . . . . . . . . . . . . . . . . . . 58

3.4 Conclusions . . . . . . . . . . . . . . . . . . 60

4 Annual VaR $\quad 65$

4.1 Introduction . . . . . . . . . . . . . . . . . . . . 65

4.2 Methodology . . . . . . . . . . . . . . . . . . 68

4.2.1 Time series models . . . . . . . . . . . . . . . . . . . 69

4.2 .2 Long Term VaR Implications . . . . . . . . . . . . . . . . . . . . . 70

4.3 Results . . . . . . . . . . . . . . . . . . . . . . 71

4.3 .1 Data . . . . . . . . . . . . . . . . 71

4.3.2 Distribution of Integrated Variance . . . . . . . . . . . . . . 74

4.3 .3 Long Term VaR . . . . . . . . . . . . . . . . . . . . . . . 78

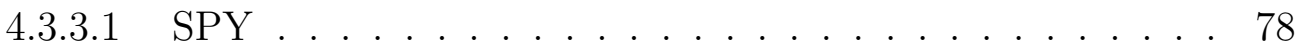

4.3.3.2 Volatility Levels . . . . . . . . . . . . . . . . 81

4.3.3.3 The Role of Persistence . . . . . . . . . . . . . . . . . . . 83

4.3.3.4 An Approximate Scaling Law . . . . . . . . . . . . . . . 83

4.3.3.5 Alternative methods and Backtesting . . . . . . . . . 86

4.4 Conclusions . . . . . . . . . . . . . . . . . . . . . . . 89

$\begin{array}{llr}5 & \text { Conclusions } & 97\end{array}$

$\begin{array}{ll}\text { References } & 99\end{array}$

Research Impact $\quad 106$

$\begin{array}{ll}\text { Biography } & 107\end{array}$ 


\section{List of Figures}

3.1 Leverage Effect $S P Y \ldots \ldots \ldots \ldots \ldots \ldots \ldots \ldots \ldots$

$3.2 \omega_{t}$ estimated from the GARCH specification $\ldots \ldots \ldots \ldots \ldots$

3.3 Probability Integral Transform Results . . . . . . . . . . . 50

$3.4 \quad$ VaR for different specifications . . . . . . . . . . . . . . 52

3.5 VaR for the time varying $\omega$ specification $\ldots \ldots \ldots \ldots \ldots$

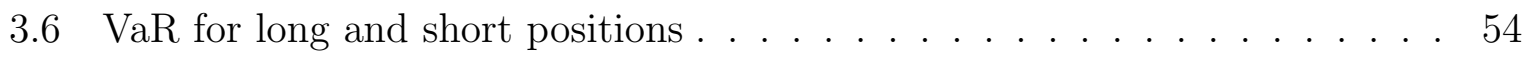

4.1 Kernel Densities of Integrated Variance under different models . . . . . . 75

4.2 Moments of the Integrated Variance distribution scaled by the horizon $\tau$. 77

4.3 Moments of the Integrated Variance distribution for different persistence levels . . . . . . . . . . . . . . . . . 77

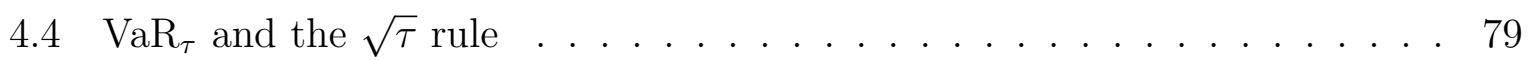

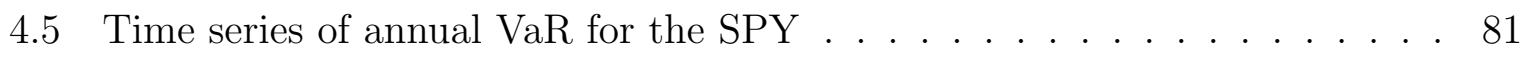

$4.6 \mathrm{VaR}_{\tau}$ at different levels of $\sigma_{t}^{2} \ldots \ldots \ldots \ldots \ldots \ldots \ldots \ldots$

4.7 Ratio of $\operatorname{VaR}_{\tau}$ obtained with two different approaches $\ldots \ldots \ldots . \ldots 8$ 


\section{List of Tables}

2.1 Parameter Estimates (SV Model) . . . . . . . . . . . . . . 16

2.2 Parameter Estimates (SVJ Model) _ . . . . . . . . . . . . 17

2.3 Parameter Estimates (SVCJ Model) . . . . . . . . . . . . . . . 18

2.4 Model Comparison via Bayes Factors … . . . . . . . . . . . . . . 19

2.5 Posterior Jump Probability on Jump vs. Non-jump Days (SVCJ Model) . 21

2.6 Index Jump Days (SVJ Model) . . . . . . . . . . . . . . . . 23

2.7 Index Jump Days (SVCJ Model) $\ldots \ldots \ldots \ldots \ldots$

2.8 Posterior Jump Probabilities and Diffusive Bounds . . . . . . . . . . 25

2.9 Jump Days with Largest and Smallest Number of Stocks Jumping (SVJ Model $\ldots \ldots \ldots \ldots \ldots \ldots \ldots . \ldots \ldots \ldots$

2.10 Signs of Individual Stock Returns on and around Index Jump Days (SVJ Model $) \ldots \ldots \ldots \ldots \ldots . \ldots \ldots$

3.1 Return Distribution . . . . . . . . . . . . . . . . . . . 44

3.2 Volatility Distribution . . . . . . . . . . . . . . . 45

$3.3 S P Y$ models' estimates . . . . . . . . . . . . . . . . . . . 46

3.4 All companies models' estimates . . . . . . . . . . . . . . . . . 48

3.5 Models' diagnostic checks for the $S P Y \ldots \ldots \ldots \ldots \ldots$

3.6 Models' diagnostic checks for all the companies in our dataset . . . . . . 51

3.7 Unconditional Coverage test $S P Y$ for Long positions . . . . . . . . . 55

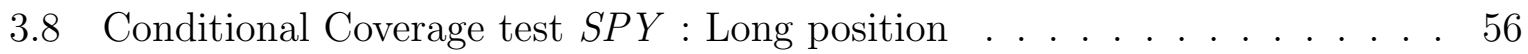

3.9 Model Confidence Set for the $S P Y$ : Long position _ . . . . . . . . 56

3.10 Unconditional Coverage test $S P Y$ : Short position . . . . . . . . 57

3.11 Model Confidence Set for the $S P Y$ : Short position _ . . . . . . 58

3.12 Robustness Statistics All Stocks: Long position _. . . . . . . . . . 59

3.13 Robustness Statistics All Stocks: Short position _. . . . . . . . . . 59

4.1 Returns Distribution . . . . . . . . . . . . . . . . . . . 72

4.2 Volatility Distribution $\ldots \ldots \ldots \ldots \ldots \ldots \ldots$

4.3 Parameter estimates . . . . . . . . . . . . . . . . 74 
4.4 Conditional moments of the Integrated Variance Distribution . . . . . . 76

4.5 VaR for $S P Y$ at different horizons . . . . . . . . . . . . . . . . 78

4.6 Estimates of the scaling parameter . . . . . . . . . . . . 80

4.7 Estimates of scaling law regression . . . . . . . . . . . . . . 85

4.8 Scalings for both models for $\alpha=0.5 \% \ldots \ldots$. . . . . . . . . 86

4.9 Performance of annual VaR measures . . . . . . . . . . . . . . . . . 88 


\section{Chapter 1}

\section{Introduction}

Financial markets are a place where households exchange today's savings for future consumption. Yet, very often the households delegate investment decisions to financial institutions such as banks, pension funds and insurance companies. The very nature of their core activities implies that their investment horizons are rather different: for instance, pension funds aim to generate wealth to guarantee a payout to future retirees and therefore their investment horizon is of 30 years and upward. Banks, on the other hand, are characterized by a much shorter investment horizon. Entering in a financial market embeds the possibility that the investor will bear a loss in the portfolio wealth due to market fluctuations. Although small size losses may be harmless, big size ones might have severe consequences for a financial institution. This is why regulators require financial institutions to compute capital requirements on the basis of a tail risk measure, an indicator of the loss under extreme market circumstances. For the banking sector, the Basel Committee has established that capital requirements must be computed using Expected Shortfall (ES) with an horizon of ten days. On the other hand, for insurance companies and pension funds, the European Insurance and Occupation Pensions Authority requires these institution to provide an annual Value-at-Risk (VaR) estimate.

This thesis contributes to tje development of methodologies for pension funds' risk management with a focus to equity risk. ${ }^{1}$ For instance, chapter 2 studies the properties of an index components and how they contribute to the index crashes. In fact, this chapter examines whether the characteristics of the index carry over to its constituents analyzing the cross-sectional heterogeneity among companies. Using jump-diffusion models to investigate this issue I find that there is a significant cross-sectional variation for the models' parameters across the index constituents. The second contribution relates these findings to abrupt negative market movements, a.k.a jumps, in the index. Most studies

\footnotetext{
${ }^{1}$ Pension funds are not only exposed to equity risk. Yet, according to a report of the International Monetary Fund, Dutch pension funds have an exposure to equity of approximately $33 \%$. Therefore, equity risk has a significant contribution to the overall risk of a pension fund.
} 
on jump detection focus on identifying jumps only in the index therefore neglecting the information coming from its constituents. This chapter focuses on systemic jumps detection by examining the index and its constituents jointly. The results show that systemic events are associated with a large number of companies exhibiting a significant negative return, which are often observed in concordance to macroeconomic news release. Identifying jumps and their drivers is a first step to improve financial institutions decision process. Yet, to protect investors' savings the development of accurate risk models is of utmost importance. Therefore the other chapters of this thesis deal with the introduction of new methodologies to compute risk measures to answer regulators' demands.

From now on and for the rest of the thesis the focus will be on VaR and comparisons with ES will be made when needed. ${ }^{2}$ For a thorough overview of different approaches to VaR estimation is given in Kuester, Mittnik, and Paolella (2006). In the following, I make a distinction between models delivering risk measures estimates using volatility proxies or alternative methods. Before introducing the models that are based on volatility, I first introduce the framework that researchers have in mind when modeling volatility. Let $p_{t}$ denotes the logarithmic price of a security, then the return is defined:

$$
r_{t}=p_{t}-p_{t-1}=\mu_{t}+\sigma_{t} \epsilon_{t}
$$

where $\mu_{t}$ denotes the mean component, $\sigma_{t}$ the volatility and $\epsilon_{t}$ a shock that has mean zero and standard deviation equal to one. The volatility component describes how the return fluctuates around its mean. Of course, a relevant role for the return dynamics is played by the stochastic component $\epsilon_{t}$. In a stylized world we might start considering that the volatility is constant, $\sigma_{t}=\sigma$ and that the shocks are standard normally distributed. Under these assumptions and setting $\mu_{t}=0$, VaR is obtained as:

$$
\operatorname{VaR}(\alpha)=\sigma Q(\alpha)
$$

with $Q(\alpha)$ denoting the standard normal quantile at probability level $\alpha$. It is then clear that under the above stated assumptions VaR is given by the product of the volatility and the quantile function of the normal distribution. Further, under the assumption that volatility is constant it follows that also the VaR is non time-varying. However, it is fairly known that volatility changes over time and therefore an unconditional VaR such as in equation (3.21) is not of high interest for short investment horizons such as one day or ten days. Therefore a vast strand of literature in finance and econometrics is dedicated to estimate a time varying measure of volatility which reflects current market conditions.

\footnotetext{
${ }^{2}$ Note that ES is a conditional expectation, where the conditioning event is that the losses are beyond the VaR level. Therefore, ES can be seen the outcome of a two step estimation procedure where the first step provides a VaR estimate.
} 
An example of this modeling approach is given in Bollerslev (1986) where the GARCH model is introduced. This model allows to have a time varying volatility depending on the recent market situation, more specifically:

$$
\sigma_{t}^{2}=\omega+\alpha \sigma_{t-1}^{2}+\beta r_{t-1}^{2}
$$

where $\omega$ denotes a constant whereas $\alpha$ and $\beta$ are the loadings on previous day variance and squared return. It readily follows that at each point in time, $t$, VaR fluctuates according to the movements in volatility. The other reason why, according to Christoffersen and Diebold (2000), this is a useful framework is given by the so called volatility clustering: periods of high volatility are likely to be followed by more high volatility periods. For a review of GARCH extensions we refer to Hansen and Lunde (2005a).

From equation (1.3) it is straightforward to notice that given information at time $t-1$ tomorrow's volatility is known as there is no stochastic component at time $t$. In contrast, when such a stochastic component is added, we are not in a conditional volatility framework anymore. This approach to volatility is known as stochastic volatility (SV) model. In this framework, the model reads:

$$
\begin{aligned}
r_{t} & =e^{\frac{h_{t}}{2}} \epsilon_{t} \\
h_{t} & =\bar{h}+\phi\left(h_{t-1}-\bar{h}\right)+\eta_{t}
\end{aligned}
$$

where $h_{t}=\ln \sigma_{t}^{2}$ and $\bar{h}$ is the long-run volatility. The main difference with GARCHlike models is then given by the stochastic component $\eta_{t}$ in (1.5). The inception of this class of models is due to Taylor (1982). For an overview of extensions of the basic SV model and their estimation we refer to Hautsch and $\mathrm{Ou}$ (2008).

The last two decades in financial econometrics have seen the rise of high-frequency data for the estimation of SV models. With the word high-frequency data we mean that return observations are available at an intra-day frequency, i.e. 5-minutes. Using these observations a vast strand of literature has focused on the estimation of volatility, which in common terminology is called realized volatility. Among these Barndorff-Nielsen and Shephard (2002a), Barndorff-Nielsen and Shephard (2002b) and Andersen, Bollerslev, Diebold, and Labys (2003) have been the first ones to study the properties of realized volatility. For instance, Barndorff-Nielsen and Shephard (2002b) and Andersen, Bollerslev, Diebold, and Labys (2003) prove the validity of realized volatility as measure of volatility. Armed with this new indicator, Andersen, Bollerslev, Diebold, and Labys (2001) and Andersen, Bollerslev, Diebold, and Ebens (2001) investigate its empirical properties: they conclude that the distribution of volatility is well described by a log-normal distribution function. Barndorff-Nielsen and Shephard (2002a) corroborate these findings concluding that 
an equally good alternative is given by the Inverse Gaussian distribution introduced in Barndorff-Nielsen (1978) and Barndorff-Nielsen (1997).

How do high-frequency data help in computing VaR? An answer is given in Andersen, Bollerslev, Diebold, and Labys (2003), where a SV model estimated using realized volatility is employed. Based on the findings in Andersen, Bollerslev, Diebold, and Labys (2001) they assume log-normality of realized volatility and that the dynamic of volatility is driven by a long memory process. ${ }^{3}$ They test this VaR strategy on exchange rate returns pointing out that it is a fruitful way to forecast the whole distribution of returns.

As explained above, in such a framework the VaR forecast is then mainly driven by the mixing distribution of volatility. To get a reasonable risk model, the mixing distribution must then have two properties: first, it must capture the volatility clustering that the data exhibit and, second, it must deliver a reasonable marginal density of return. Therefore, chapter 3 focuses on the properties of the volatility shocks rather than the conditional volatility. ${ }^{4}$ Understanding the properties of the shocks is fundamental in this modeling strategy as they characterize the return density and therefore the VaR forecast. This chapter assesses what is a good mixing density for Realized Volatility and what are the implications for VaR forecasting.

When it comes to estimating VaR there exist methods who are not solely based on volatility. An example of this is given by Extreme Value Theory (EVT), where the main focus is the direct modeling of the tail of the return distribution, for a detailed introduction I refer to Danielsson and De Vries (2000). Yet, EVT is based on the assumption that observations are independent over time, which under the paradigm given by equation (1.1) is not the case. Also, it is worth highlighting that under the basic EVT the VaR estimate is an unconditional measure and it does not change with recent market conditions. To overcome the problem with the independence assumption McNeil and Frey (2000) develops a new model where EVT is applied to the filtered return shocks. Their method is based on two steps: in the first one, the shocks are filtered by means of a GARCH model, then EVT is applied to these innovations. They conclude that a conditional approach delivers better assessment of tail risk than an the unconditional EVT approach. On this idea, the work of Bee, Dupuis, and Trapin (2016) adds high-frequency to this method. More specifically, they use several forecasting models for realized volatility to filter the innovations in the returns equation. Their results point out that using high frequency data delivers better

\footnotetext{
${ }^{3}$ For a detailed introduction to long memory processes we refer to Baillie (1996).

${ }^{4}$ The time series specification used is the Heterogeneous AutoRegressive (HAR) model introduced in Corsi (2009), which captures volatility clustering by means of a restricted autoregressive process of order 22. Since its introduction, this has been extended to capture other features such as jumps, leverage and time varying measurement error. For these extensions we refer to Andersen, Bollerslev, and Diebold (2005), Patton and Sheppard (2015), Corsi and Renò (2012) and Bollerslev, Patton, and Quaedvlieg (2016).
} 
risk assessments compared to the conditional EVT introduced in McNeil and Frey (2000). So far the main methodologies for a daily VaR have been reviewed, yet as mentioned above for pension funds, the regulator ask for an annual VaR estimate. More precisely, EIOPA require pension funds and insurance companies to provide an annual VaR estimate but leave some flexibility to each country's regulator in the refinement of these capital requirements. For instance, focusing on the Dutch case, the supervisor and regulator for financial institutions in the Netherlands is De Nederlandsche Bank (DNB). DNB imposes the legal requirement that the pension fund must have sufficient capital to ensure that there is a probability of $97.5 \%$ that the market value of the assets will not decline below the market value of the liabilities. Yet, DNB leaves the pension fund three options to provide an annual VaR estimate. The first approach is given by the standard model. This approach is directly borrowed by EIOPA's directives. For the equity risk submodule, the regulator suggests to apply a shock of $40 \%$ as a prudent estimate. The second approach allows the pension fund to partially adopt the standard model and an internally developed one. Finally, the third case allows the pension fund to compute VaR with a fully internally developed one, once it has been approved by DNB.

Therefore, the properties of annual return are of utmost importance in developing a model that needs approval from the regulator. From a more formal perspective when returns aggregate we want to understand the properties of the cumulative return over the investment horizon $\tau$, say one year, defined as:

$$
R_{t+\tau}=\sum_{i=1}^{\tau} r_{t+i}
$$

and $r_{t+i}$ follows from the framework given in (1.1). When dealing with an annual horizon studying the properties of the cumulative returns in an accurate manner becomes more difficult due to the fact that the number of non-overlapping observations shrinks with $\tau$. For example, EIOPA requires pension funds and insurance companies to provide a $99.5 \%$ VaR estimate, which corresponds to an expected violation in two hundred years. With this limited number of returns observations at our disposal this is simply impossible. This is why ad hoc methods need to be developed and employed to provide an estimate of the $99.5 \%$ VaR. For instance, in order to obtain the $40 \%$ estimate in the EIOPA directive, a pension fund should aggregate daily return and have an overlap between two consecutive observations of one year. This way, the resulting sample size would be $T$, the number of daily return, minus the overlap length, one year in this case. Once, this new set of dependent annual return is obtained the sample moments are estimated and plugged in the VaR formula. Of course, in this case the magnitude of the VaR does not reflect current market information. In chapter 4 I investigate the usefulness 
of a stochastic volatility model calibrated on High-Frequency data to provide annual VaR estimate. In this chapter I highlight that also for an annual horizon, VaR should be computed considering the most recent market information, reflected in the volatility level. In fact, as pointed out previously, one of the characteristics of volatility is the property to stay at a specific level, either high or low, for a long time span.

Another approach to estimating multi-period VaR is with the usage of a scaling law. In this case, an estimate of the $\tau$ period VaR is obtained through the simple relation:

$$
\operatorname{VaR}_{\tau}(\alpha)=\tau^{\frac{1}{c}} \operatorname{VaR}_{1}(\alpha)
$$

As equation (1.7) shows, a scaling law links the one-day VaR to the $\tau$ period counterpart by a scaling factor, which depends on the horizon length $\tau$. A special case of a scaling law is given by the square root of time rule, which arises if $c=2$ and it would be correct if the daily return is iid normally distributed.

Another scaling law is provided by Extreme Value Theory (EVT). In this case the scaling parameter $c$ corresponds to the tail index, $\xi$, which measures how heavy tailed the return distribution is. Empirical studies find estimate the tail index between three and four as shown in Danielsson and De Vries (2000) and therefore implies, ceteris paribus, a less conservative annual VaR estimate than the square root of time rule.

In chapter 4, an approximate scaling law is obtained. Like other studies in the literature, such as McNeil and Frey (2000) and Danielsson and Zigrand (2006), I show that an appropriate scaling law should be of order higher than implied by the square root of time rule. 


\section{Chapter 2}

\section{A Jumping Index of Jumping Stocks?*}

\section{$2.1 \quad$ Introduction}

This paper analyses continuous time jump diffusion models for single stock returns. In particular, we are interested in the question of how the dynamics for index constituents differ from the index, and whether a jump in the index is triggered by jumps in the constituents. By answering these questions we contribute to the understanding of return dynamics in stock markets.

The analysis of the statistical properties of stock returns is one of the main points of inquiry in empirical finance research. There is a large body of literature that focuses on continuous-time models designed to capture essential features of observed stock price movements, including time-varying variance and jumps, i.e., sudden large movements in prices. Empirical testing of these continuous time equity price models has been at the center of many empirical studies. Starting with models with stochastic volatility (see, e.g. Jacquier, Polson, and Rossi (1994) and Jacquier, Polson, and Rossi (2004)) the literature has evolved to models with jump components in returns (see, e.g. Bakshi, Cao, and Chen (1997) and Pan (2002)) and to models containing jumps in returns and in volatility (see, among others, Eraker, Johannes, and Polson (2003), Eraker (2004), Broadie, Chernov, and Johannes (2007)). ${ }^{2}$

\footnotetext{
${ }^{*}$ This chapter is based on joint work with Paulo Rodrigues, Christian Schlag and Norman Seeger.

${ }^{2}$ Recently, it has been shown that to further improve the ability of a model to consistently reproduce stylized facts in the data, it seems helpful to include non-affine terms in the variance process. Examples of papers from this strand of the literature are Christoffersen, Jacobs, and Mimouni (2010), Chourdakis and Dotsis (2011), Mijatovic and Schneider (2014), and Ignatieva, Rodrigues, and Seeger (2015). Nevertheless, for the analysis in this paper we opt to analyze only affine jump diffusion models. We do so for a number of reasons. First, we learn from the above papers that the jump components for non-affine specifications usually do not differ substantially from those for affine models, and since jumps are the key object of our investigation, we do not expect to gain much from using non-affine models. Second, building on the latter
} 
Empirical investigations of equity asset pricing models have focused on the major equity market indices like the S\&P 500 or the NASDAQ 100 as the main economic object of investigation. From the literature one can identify certain characteristics of stock price models that can be termed as stylized facts. First, models with just a stochastic volatility component, but without jumps, appear to be significantly mis-specified. Second, when jumps are included in the model, they turn out to be rare, negative and large in absolute value. Third, there is a negative correlation between the innovations in the return and the variance process. Since all these findings were made exclusively for indices, the big question is if they also remain valid for individual stocks. Answering this question represents the major motivation for our paper.

In the first step of our analysis we compare estimated model parameters for the S\&P 100 index with the parameters of the single stock constituents. We employ a pure stochastic volatility model (SV), an SV including jumps in prices (SVJ), and an SV model featuring correlated jumps in the stock price and the conditional variance (SVCJ). Estimation of the models analyzed is based on the Markov Chain Monte Carlo (MCMC) approach developed in Eraker, Johannes, and Polson (2003). At the center of our study lies the analysis of index versus single stock jumps. For this reason, we take an additional step of running a simulation study on the jump models considered in our setup to gain insights on how to properly identify jump days. The simulation study provides us with a posterior jump probability that allows us to cleanly differentiate between jump days and non-jump days. In the main body of our study we analyze the relationship of index jumps and how they are related to jumps in the single stock constituents of the index.

Our first set of results shows that the stochastic process for the 'typical' (i.e., average) individual stock is significantly different than for the market index, in our case represented by the S\&P 100. The average size of the jumps in the stock price is smaller in absolute terms than for the index, and jumps in individual stock prices are even in many cases on average positive. Although jumps are still somewhat rare, the frequency of jumps in prices is more than five times as high for the representative stock than the index, Thus, jumps tend to occur much more frequently for the average stock than for the S\&P 100. Furthermore, the correlation between stock returns and volatility changes is estimated at values that are much less negative than for the index. This last result is, of course, derived under the physical probability measure, but is nevertheless related to empirical findings concerning the pricing of options on indices and individual stocks. As shown by Bollen and Whaley (2004) the implied volatility curves for stock market indices tend to be negatively sloped and much steeper than those for the index component

argument, we do not want to introduce an additional layer of complexity by using non-affine models, but rather go with the cleanest possible setup while still being able to analyze our research question. 
stocks. Also Bakshi, Kapadia, and Madan (2003), Dennis and Mayhew (2002) and Dennis, Mayhew, and Stivers (2006) provide evidence for structural differences in the pricing of index and individual stock options. In line with these results from empirical option pricing research, our findings support the notion that one cannot simply extend the results from the analyses of major equity indices to single stocks.

The main contribution of our empirical analysis concerns the behavior of the individual stocks on days when the index exhibits a jump. Surprisingly, we find index jump days where relatively few stocks also exhibit jumps. For the SVJ model we find days (like February 24, 1994, or January 4, 2000) where the number of jumping stocks is 1 . On the other extreme, again for the SVJ model, the highest number of such contemporaneous stock jumps is 75 on October 13, 1989, and 63 observed on October 27, 1997. These findings immediately raise the question of whether the models estimated for the index and the individual stocks are compatible at all. The solution to this apparent 'puzzle' can be found via a detailed analysis of the mechanics of the models employed in our study. A jump is considered likely (in terms of posterior probability) if the price move of an asset on a given day is large relative to the conditional variance. The index as a diversified portfolio of stocks then naturally jumps on days when most of the component stocks exhibit large returns (but not necessarily jumps) in the same direction. Since the average local variance of the stocks in our sample is fairly stable around index jump days, at least some of these jumps must be caused by a substantial increase in the correlation between the index component stocks, generating the large and negative index return, which is then identified as a jump. We label these jumps as 'correlation jumps'. The remaining jumps, which we call 'macro-driven jumps', are more interesting from an economic point of view. They are characterized by a large number of stocks or sectors exhibiting high jump probabilities simultaneously, and we can clearly link these jumps to important macroeconomic events. A further surprising result of our analysis is that jump models unexpectedly do not identify an unusually large number of jumps during the time of the financial crisis in 2008. This is surprising since 13 of the 20 largest daily return movements in our sample occurred during the financial crisis period. We show that this can be explained by the fact that during the financial crisis we observe a prolonged period of high volatility and the large return movements during that time were within two standard deviations of the diffusive volatility component. This implies that these large return movements can be generated by a pure stochastic volatility model without relying on jumps.

An important overall conclusion we draw from our analysis concerning the 'right' model for the index itself and its constituents is the following: Even if a pure stochastic volatility model could sufficiently represent the dynamics of the index constituents (single stocks), there would still be strong evidence for jumps in the index dynamics, so that here a jump 
model seems appropriate.

A study that is related to our paper in terms of analyzing the properties of individual stock returns is Maheu and McCurdy (2004), who estimate the parameters of a discretetime GARCH model with jumps via maximum likelihood for a rather small set of selected stocks. They attribute jumps to corporate news events and show that this hypothesis is supported by the data. In contrast to their approach, we consider a richer set of models by using a SV, a SVJ, and a SVCJ model, and investigate these models for the large cross-section of stocks constituting the S\&P 100 index. Jiang and Yao (2013) employ a methodology developed in Barndorff-Nielsen and Shephard (2004) and analyze a long time series of stock returns to decompose price changes into a continuous and a jump part. Their focus is on cross-sectional return predictability characteristics like size and book-tomarket, and their results show that a large part of this predictability is due to differences in the jump part of returns. Şerban, Lehoczky, and Seppi (2008) propose a model where stock returns are driven by a market factor, a 'common idiosyncratic' component and a factor which is truly idiosyncratic to the respective stock. Their findings indicate that the common factor in idiosyncratic volatility is relevant for option pricing. A more recent strand of literature examines co-jumps between stock returns. Examples can be found in Caporin, Kolokolov, and Renó (2017) and Gilder, Shackleton, and Taylor (2014). While the papers just mentioned investigate the relationship between single stocks, our paper focuses on the relationship between the stock index and its constituents.

The remainder of the paper is structured as follows. In Section 2.2 we present the model and describe our estimation approach. The results are then discussed in Section 2.3. Section 2.4 concludes.

\subsection{Model and Estimation Approach}

\subsubsection{Model}

Our model specification follows Eraker, Johannes, and Polson (2003). Their approach provides a flexible model structure, which allows for jumps both in prices and the conditional volatility process. The logarithm of the stock price $(Y)$ and the variance $(V)$ are assumed to follow the continuous-time processes 


$$
\begin{aligned}
& d Y_{t}=\mu d t+\sqrt{V}_{t} d W_{t}^{y}+d\left(\sum_{j=1}^{N_{t}} \xi_{j}^{y}\right) \\
& d V_{t}=\left(\alpha_{0}+\alpha_{1} V_{t}\right) d t+\sigma_{v} \sqrt{V_{t}} d W_{t}^{v}+d\left(\sum_{j=1}^{N_{t}} \xi_{j}^{v}\right)
\end{aligned}
$$

where $d W_{t}^{y}$ and $d W_{t}^{v}$ denote Brownian increments with correlation $E\left(d W_{t}^{y} d W_{t}^{v}\right)=$ $\rho d t$. The fact that there is a (empirically mostly negative) correlation between returns and variance innovations is often called the 'leverage effect'. The term $\mu$ captures the expected return, and $N_{t}$ denotes a Poisson process with constant intensity $\lambda$. The Poisson process enters both the return and variance equation, thus generating simultaneous jumps. $\xi^{y}$ and $\xi^{v}$ denote the jump sizes in returns and variance, respectively, and these jump sizes can be correlated. In more detail, the jump size in variance follows an exponential distribution with parameter $\mu_{v}^{-1}$, i.e., $\xi_{t}^{v} \sim \operatorname{Exp}\left(\mu_{v}^{-1}\right)$ with expected value and standard deviation equal to $\mu_{v}$. While conditional on $\xi_{t}^{v}$, the jump size in the log price at time $t$ follows a normal distribution with mean $\mu_{y}+\rho_{j} \xi_{t}^{v}$ and variance $\sigma_{y}^{2}$, i.e., $\xi_{t}^{y} \mid \xi_{t}^{v} \sim N\left(\mu_{y}+\rho_{j} \xi_{t}^{v}, \sigma_{y}^{2}\right)$. The variance process follows an affine structure. For the SV model the long-term mean is given by $-\alpha_{0} / \alpha_{1}$, whereas for a jump model we have a long-term mean of $\left(-\alpha_{0}-\lambda \mu_{v}\right) / \alpha_{1}$. The speed of mean reversion given by $-\alpha_{1}$.

The general case represented by the dynamics in Equations (2.1) and (2.2) is called the stochastic volatility model with contemporaneous jumps in the return and the conditional variance (SVCJ model). This is the model that performed best in the analysis by Eraker, Johannes, and Polson (2003). Besides the general model we also consider two special cases. By setting $\lambda=0$, the SVCJ model reduces to the well-known stochastic volatility (SV) model proposed by Heston (1993). If we include jumps, but only in returns, we obtain the stochastic volatility model with jumps (SVJ) first considered by Bates (1996). In this case we restrict the jump size in volatility to be identically equal to zero $\xi^{v} \equiv 0$, implying that jumps in returns are normally distributed with mean $\mu_{y}$ and variance $\sigma_{y}^{2}$.

To estimate the models, we use an Euler discretization scheme and set the interval at $\Delta=1$ (day). Denoting the log return of the asset $Y_{t}-Y_{t-1}$ by $R_{t}$, we can write the discretized version of the system in (2.1) and (2.2) as

$$
\begin{aligned}
R_{t} & =\mu+\sqrt{V_{t-1}} \varepsilon_{t}^{y}+\xi_{t}^{y} J_{t} \\
V_{t} & =V_{t-1}+\alpha_{0}+\alpha_{1} V_{t-1}+\sigma_{v} \sqrt{V_{t-1}} \varepsilon_{t}^{v}+\xi_{t}^{v} J_{t}
\end{aligned}
$$


where shocks to returns and volatility, $\varepsilon_{t}^{y}=W_{t}^{y}-W_{t-1}^{y}$ and $\varepsilon_{t}^{v}=W_{t}^{v}-W_{t-1}^{v}$, follow a bivariate normal distribution with zero expectation, unit variance, and correlation $\rho$. In the Euler discretization scheme, we assume, at most, one jump per day. This is not a problem, since, given the observation frequency, we cannot distinguish anyway between situations where multiple (smaller) jumps or only one larger jump have occurred during a day . $J_{t}$, thus, represents an indicator that is set equal to one in the case of a jump and to zero otherwise. Due to the assumption of contemporaneous jumps in returns and volatility this indicator is of course the same in the dynamics for $R$ and $V$. The jump sizes retain the distributional assumptions described above.

For technical details concerning the above discretization schemes the reader is referred to Jones (2003b), Eraker, Johannes, and Polson (2003), Jones (2003a), Ait-Sahalia (1996), and Conley, Hansen, Luttmer, and Scheinkman (1997).

\subsubsection{Estimation Approach}

The underlying model setup includes latent variables such as volatility, jump times, and jump sizes. Each of these latent states are treated as a parameter to be estimated in a Bayesian context. This leads to a high dimensional posterior distribution, which is not equal to a known statistical distribution. We therefore rely on Markov Chain Monte Carlo (MCMC) techniques to compute the posterior moments. We report the posterior means and the posterior standard deviations in the estimation results.

In a nutshell, MCMC allows us to draw from a high dimensional distribution by breaking it down into draws from a series of lower dimensional conditional distributions. ${ }^{3}$ We are, thus, able to construct a Markov Chain that converges to the desired posterior distribution. After convergence, we draw $N$ times from that posterior to perform Monte Carlo integration. ${ }^{4}$ In the following, we provide a brief overview of the algorithm by using the SVCJ model specification since this yields the most complex structure. For more details on the sampling algorithm we refer to Ignatieva, Rodrigues, and Seeger (2015).

According to Bayes Theorem, the posterior distribution of the parameters and the latent states is proportional to the likelihood times the prior distribution

$$
p\left(\boldsymbol{\Theta}, \boldsymbol{V}, \boldsymbol{\xi}^{y}, \boldsymbol{\xi}^{v}, \boldsymbol{J} \mid \boldsymbol{R}\right) \propto p\left(\boldsymbol{R} \mid \boldsymbol{V}, \boldsymbol{\xi}^{y}, \boldsymbol{\xi}^{v}, \boldsymbol{J}, \boldsymbol{\Theta}\right) p\left(\boldsymbol{V}, \boldsymbol{\xi}^{y}, \boldsymbol{\xi}^{v}, \boldsymbol{J}, \boldsymbol{\Theta}\right)
$$

\footnotetext{
${ }^{3}$ For a detailed discussion of this algorithm in a financial econometrics context, see Johannes and Polson (2006).

${ }^{4}$ In our empirical analysis we use a burn-in period of 600,000 and then draw 1.4 million times from the posterior distribution. This large number of draws is necessary to ensure convergence in the estimation of the polynomial models. The remaining models will converge after a much smaller number of overall draws.
} 
where we denote by $\boldsymbol{\Theta}=\left(\mu, \alpha\left(V_{t}\right), \gamma\left(V_{t}\right), \rho, \mu_{y}, \sigma_{y}, \rho_{j}, \mu_{v}, \lambda\right)^{\top}$ the vector of model parameters, with the expression $\alpha\left(V_{t}\right)$ denoting the parameters entering the drift specifications, and $\gamma\left(V_{t}\right)$ denoting the parameters entering the diffusion specification of the variance. The time series of state variables is collected into $\left\{\boldsymbol{V}, \boldsymbol{\xi}^{y}, \boldsymbol{\xi}^{v}, \boldsymbol{J}\right\}$, and $\boldsymbol{R}$ denotes the time series of observed returns. Note that our model specifications allow us to give the prior a hierarchical structure. Therefore,

$$
p\left(\boldsymbol{V}, \boldsymbol{\xi}^{y}, \boldsymbol{\xi}^{v}, \boldsymbol{J}, \boldsymbol{\Theta}\right)=p\left(\boldsymbol{V} \mid \boldsymbol{\xi}^{v}, \boldsymbol{J}, \boldsymbol{\Theta}\right) p\left(\boldsymbol{\xi}^{y} \mid \boldsymbol{\xi}^{v}, \boldsymbol{\Theta}\right) p\left(\boldsymbol{\xi}^{v} \mid \boldsymbol{\Theta}\right) p(\boldsymbol{J} \mid \boldsymbol{\Theta}) p(\boldsymbol{\Theta})
$$

Given our model framework, the only component of this prior distribution not determined by the model is the component given by $p(\boldsymbol{\Theta})$. We use the same set of independent conjugate priors described in Ignatieva, Rodrigues, and Seeger (2015). Given the Markov property of the model we can rewrite the remaining components of the posterior distribution as follows

$$
\begin{aligned}
& p\left(\boldsymbol{R} \mid \boldsymbol{V}, \boldsymbol{\xi}^{y}, \boldsymbol{\xi}^{v}, \boldsymbol{J}, \boldsymbol{\Theta}\right)=\prod_{t=1}^{T} p\left(R_{t} \mid V_{t}, V_{t-1}, \xi_{t}^{y}, \xi_{t}^{v}, \boldsymbol{\Theta}\right) \\
& p\left(\boldsymbol{V} \mid \boldsymbol{\xi}^{v}, \boldsymbol{J}, \boldsymbol{\Theta}\right) \propto \prod_{t=1}^{T} p\left(V_{t} \mid V_{t-1}, \xi_{t}^{v} J_{t}, \boldsymbol{\Theta}\right) \\
& p\left(\boldsymbol{\xi}^{y} \mid \boldsymbol{\xi}^{v}, \boldsymbol{\Theta}\right)=\prod_{t=1}^{T} p\left(\xi_{t}^{y} \mid \xi_{t}^{v}, \boldsymbol{\Theta}\right) \\
& p\left(\boldsymbol{\xi}^{v} \mid \boldsymbol{\Theta}\right)=\prod_{t=1}^{T} p\left(\xi_{t}^{v} \mid \boldsymbol{\Theta}\right) \\
& p(\boldsymbol{J} \mid \boldsymbol{\Theta})=\prod_{t=1}^{T} p\left(J_{t} \mid \boldsymbol{\Theta}\right)
\end{aligned}
$$

The MCMC sampler now samples iteratively through the following complete conditional distributions

$$
\begin{array}{cl}
\text { Parameters : } & p\left(\boldsymbol{\Theta}_{i} \mid \boldsymbol{\Theta}_{-i}, \boldsymbol{J}, \boldsymbol{\xi}^{y}, \boldsymbol{\xi}^{v}, \boldsymbol{V}, \boldsymbol{R}\right), i=1, \ldots, K \\
\text { Jump times : } & p\left(J_{t} \mid \boldsymbol{\Theta}, \boldsymbol{J}_{-t}, \boldsymbol{\xi}^{y}, \boldsymbol{\xi}^{v}, \boldsymbol{V}, \boldsymbol{R}\right), t=1, \ldots, T \\
\text { Jump sizes : } & p\left(\xi_{t}^{y} \mid \boldsymbol{\Theta}, \boldsymbol{J}, \boldsymbol{\xi}_{-t}^{y}, \boldsymbol{\xi}^{v}, \boldsymbol{V}, \boldsymbol{R}\right), t=1, \ldots, T \\
& p\left(\xi_{t}^{v} \mid \boldsymbol{\Theta}, \boldsymbol{J}, \boldsymbol{\xi}_{-t}^{v}, \boldsymbol{\xi}^{y}, \boldsymbol{V}, \boldsymbol{R}\right), t=1, \ldots, T \\
\text { Volatility : } & p\left(V_{t} \mid \boldsymbol{\Theta}, V_{t+1}, V_{t-1}, \boldsymbol{J}, \boldsymbol{\xi}^{v}, \boldsymbol{\xi}^{y}, R_{t+1}, R_{t}\right), t=1, \ldots, T
\end{array}
$$


where we denote the $i$-th element of a vector by $\boldsymbol{\Theta}_{i}$. The remaining parameters in a vector excluding a particular parameter $i$ is indicated by a minus sign in front of the index. That is, $\boldsymbol{\Theta}_{-i}$ indicates the elements of the vector without the $i$-th element.

By relying on conjugate priors for the model parameters, we are able to use a Gibbs steps for updating all parameters, jump times, and jump sizes. The only parameters not having a recognizably complete conditional distribution are the variances, denoted by $V_{t}$ above. The complete conditional distribution for $V_{t}$ is given by

$p\left(V_{t} \mid \boldsymbol{\Theta}, V_{t+1}, V_{t-1}, \boldsymbol{J}, \boldsymbol{\xi}^{v}, \boldsymbol{\xi}^{y}, R_{t+1}, R_{t}\right)=p\left(R_{t+1}, V_{t+1} \mid \boldsymbol{\Theta}, V_{t}, \boldsymbol{J}, \boldsymbol{\xi}^{y}, \boldsymbol{\xi}^{v}\right) p\left(V_{t} \mid \boldsymbol{\Theta}, R_{t}, V_{t-1}, \boldsymbol{J}, \boldsymbol{\xi}^{y}, \boldsymbol{\xi}^{v}\right)$

where the first component on the right-hand side of the equation denotes a bivariate normal distribution and the second component denotes a univariate normal distribution. The Metropolis-Hastings step proposes a new variance $V_{t}^{(g)}$ in iteration $g$ by drawing from $p\left(V_{t} \mid \boldsymbol{\Theta}, R_{t}, V_{t-1}, \boldsymbol{J}, \boldsymbol{\xi}^{y}, \boldsymbol{\xi}^{v}\right)$ and accepting that draw with probability

$$
\min \left\{\frac{p\left(R_{t+1}, V_{t+1} \mid \boldsymbol{\Theta}, V_{t+1}, V_{t}^{(g)}, \boldsymbol{J}, \boldsymbol{\xi}^{y}, \boldsymbol{\xi}^{v}\right)}{p\left(R_{t+1}, V_{t+1} \mid \boldsymbol{\Theta}, V_{t+1}, V_{t}^{(g-1)}, \boldsymbol{J}, \boldsymbol{\xi}^{y}, \boldsymbol{\xi}^{v}\right)}, 1\right\}
$$

which shows that the proposed candidate draws are consistent with $V_{t-1}$ and $R_{t}$ and accepted consistent with $V_{t+1}$ and $R_{t+1}$.

\subsection{Empirical Analysis}

\subsubsection{Data}

Our stock return data for the period from 1980 to 2014 is obtained from the Center for Research in Security Prices (CRSP) database. We use the index constituents file from Compustat to identify the companies included in the S\&P 100 index on any given day in the sample. Although the launch date for the S\&P 100 index is June 15, 1983, Compustat only provides information on index constituents beginning in September 1989. Therefore, we begin our analysis of index jump days from that date.

We find a total of 205 companies that were at one point in time in the S\&P 100 index, we matched these companies with the return information provided by CRSP using the cusip identifier. From the 205 companies, using the cusip identifier we are able to unambiguously match 201. These 201 companies are used in our analysis. On a daily basis, we can match between 92 to 99 stocks with the 94 to 100 stocks listed in the index 
by Compustat . This indicates that we are able to almost completely replicate the index constituents with our sample.

\subsubsection{Parameter Estimates and Model Choice}

We will first discuss the parameter estimates for the different models. Our estimation results for the S\&P 100 index are comparable, as expected, to those shown in other papers for indices like the S\&P 500. This result ensures that the differences between the index constituents and the index can be carried over to a more widely used index like the S\&P 500, and are not due to our index selection.

We are interested in the differences of the parameter estimators for the single stocks and the stock index constituted by these stocks. In particular, the jump parameters are at the center of our analysis, as well as, the posterior model odds comparing a pure stochastic volatility model to the jump diffusion specifications.

\subsubsection{SV}

In Column 1 of Table 2.1 we can see that, as in basically all empirical studies for the Heston SV model, the correlation between diffusive price changes and diffusive volatility changes is strongly negative for the index with a value of roughly -0.6 . For the typical stock, however, $\rho$ is much less negative with a cross-sectional average estimate of -0.27 with $95 \%$ percent of the estimates ranging between -0.51 and -0.06 . Although we do not analyze options data in this paper, this result for $\rho$ provides support (under the $\mathbb{P}_{-}$ measure) for the finding that implied volatility smiles for individual stocks tend to be much flatter than those for the major equity indices around the world (see, e.g. Bollen and Whaley (2004)).

Not surprisingly, the parameter estimates for the single stocks imply a widely differing dynamic structure for the single stocks . The variance parameter $\alpha_{0}$ has a mean of 0.0264 for the index whereas the $95 \%$ quantile for the single stocks ranges from 0.0367 to 0.3759 . The parameter $\alpha_{1}$ ranges from -0.0941 to -0.0061 , with the value for the index being 0.0216. These parameter values imply large differences in long run mean or speed of mean reversion for the index and the single stocks. For the long run mean of volatility we have an approximate average value of $35 \%$ annually for the single stocks versus $17.5 \%$ for the index. However, since the parameter $\alpha_{1}$ is negative we have mean reverting variances for all return series. 
Table 2.1: Parameter Estimates (SV Model)

\begin{tabular}{crrrrrrrr}
\hline & \multicolumn{2}{c}{ Index } & & \multicolumn{4}{c}{ Individual Stocks } \\
\cline { 2 - 3 } \cline { 5 - 7 } \cline { 5 - 7 } & \multicolumn{1}{c}{ mean } & std. err. & & mean & std. dev. & $2.5 \%$ & $97.5 \%$ \\
\hline$\mu$ & 0.0305 & 0.0087 & & 0.0281 & 0.0382 & -0.0365 & 0.0930 \\
$\alpha_{0}$ & 0.0264 & 0.0027 & & 0.1376 & 0.1179 & 0.0367 & 0.3759 \\
$\alpha_{1}$ & -0.0216 & 0.0026 & & -0.0299 & 0.0225 & -0.0941 & -0.0061 \\
$\sigma_{v}$ & 0.1791 & 0.0082 & & 0.3722 & 0.1318 & 0.2043 & 0.6507 \\
$\rho$ & -0.5899 & 0.0322 & & -0.2673 & 0.1095 & -0.5127 & -0.0574 \\
\hline
\end{tabular}

NOTE: The table shows the estimated parameters for the index (posterior mean and standard error) and cross-sectional summary statistics for the posterior means of the parameter estimates for individual stocks. An overview of the stocks included in our sample and the respective estimation period can be found in the online appendix.

\subsubsection{SVJ}

The SVJ model, in contrast with the SV model, introduces jumps in the price process. The parameter estimates are reported in Table 2.2. The estimates for $\alpha_{0}, \alpha_{1}$, and $\rho$, as well as the differences between the index and the single stocks, are very similar to the SV case, so we will not discuss them in detail.

One of the key parameters in the SVJ model is the mean jump size. A stylized fact from empirical research is that this quantity is negative and large in absolute value for the major equity indices. This is confirmed in our analysis, since $\mu_{y}$ for the S\&P 100 is estimated at -2.37 with a standard error of 0.87 , i.e., significantly different from zero. In contrast to this, the typical stock exhibits a weakly positive expected jump size (0.79) with a very large cross-sectional variation with $2.5 \%$ - and $97.5 \%$-quantiles equal to -1.11 and 3.37, respectively. Looking deeper into the results for the single stocks, we find examples of both significantly positive and significantly negative jump sizes, again evidence for the wide variation in the characteristics of the stochastic processes for the stocks in our sample. These findings clearly show that the estimation results for indices cannot be generalized to individual stocks and that there is no 'law' that jump sizes can only be chosen to be negative in applications of SVJ models. As one might expect, the estimated standard deviation of the jump size $\sigma_{y}$ is much smaller for the index (2.76) than for the average stock (5.38). Again this is evidence for the large cross-sectional variation across stocks.

Another key parameter of a jump process is the intensity, or loosely speaking, the probability of a jump over the next time interval (here, one day). Again, the differences between the stocks and the index are striking. For the S\&P 100, $\lambda$ is estimated at 0.0051 
Table 2.2: Parameter Estimates (SVJ Model)

\begin{tabular}{crrrrrrrr}
\hline & \multicolumn{2}{c}{ Index } & & \multicolumn{4}{c}{ Individual Stocks } \\
\cline { 2 - 3 } \cline { 5 - 7 } \cline { 5 - 7 } & mean & std. err. & & mean & std. dev. & $2.5 \%$ & $97.5 \%$ \\
\hline$\mu$ & 0.0325 & 0.0087 & & 0.0175 & 0.0596 & -0.0803 & 0.0829 \\
$\alpha_{0}$ & 0.0219 & 0.0025 & & 0.0657 & 0.0936 & 0.0097 & 0.1699 \\
$\alpha_{1}$ & -0.0181 & 0.0023 & & -0.0180 & 0.0281 & -0.0500 & -0.0016 \\
$\sigma_{v}$ & 0.1625 & 0.0081 & & 0.2322 & 0.0861 & 0.1322 & 0.3577 \\
$\rho$ & -0.6418 & 0.0297 & & -0.3476 & 0.1256 & -0.6194 & -0.0650 \\
$\mu_{Y}$ & -2.3672 & 0.8727 & & 0.7901 & 1.0811 & -1.1148 & 3.3742 \\
$\sigma_{Y}$ & 2.7602 & 0.6041 & & 5.3834 & 3.6092 & 1.7476 & 14.3364 \\
$\lambda$ & 0.0051 & 0.0021 & & 0.0360 & 0.0431 & 0.0057 & 0.1026 \\
\hline
\end{tabular}

NOTE: The table shows the estimated parameters for the index (posterior mean and standard error) and cross-sectional summary statistics for the posterior means of the parameter estimates for individual stocks. An overview of the stocks included in our sample and the respective estimation period can be found in the online appendix.

corresponding to an expected number of roughly 1.26 jumps per year. For the average stock, $\lambda$ is estimated to be about seven times larger (0.0360). Again there is pronounced cross-sectional variation in this estimate for individual stocks with a $2.5 \%$-quantile of 1.43 jumps per year, while the stock representing 97.5\%-quantile would, on average, exhibit 25.9 jumps annually.

\subsubsection{SVCJ}

The SVCJ model (in contrast with the SVJ model) includes jumps in the local variance process $V$. In Table 2.3, we see again that jumps are rare events for the index with an estimate for $\lambda$ of 0.0057 , implying about 1.4 jumps per year. For the stocks, we obtain a cross-sectional average for $\lambda$ of 0.0267 , resulting in an average number of 6.7 jumps annually. The SVCJ model is similar to the models analyzed above in that there is considerable variation in these estimates across stocks with $95 \%$ of the estimates ranging between roughly 1 and 19 jumps per year.

Similar to the other models, the correlation between diffusive price changes and diffusive volatility changes is strongly negative for index (roughly -0.66) and much less negative for the typical stock, with a cross-sectional average estimate of -0.37 . Nevertheless, this correlations tends to be negative also for the stocks, with a cross-sectional $97.5 \%$-quantile of -0.09 .

One has to be careful when interpreting the expected jump size for the price process. Conditional on the current jump size in volatility it is given by $\mu_{y}+\rho_{j} \xi_{t}^{V}$, which yields an 
Table 2.3: Parameter Estimates (SVCJ Model)

\begin{tabular}{lrrrrrrr}
\hline & \multicolumn{2}{c}{ Index } & & \multicolumn{4}{c}{ Stocks } \\
\cline { 2 - 3 } \cline { 6 - 7 } & Mean & std. err. & & Mean & std. err. & $2.5 \%$ & $97.5 \%$ \\
\hline$\mu$ & 0.0365 & 0.0087 & & 0.0238 & 0.0457 & -0.0736 & 0.0837 \\
$\alpha_{0}$ & 0.0215 & 0.0025 & & 0.0605 & 0.1032 & 0.0059 & 0.2540 \\
$\alpha_{1}$ & -0.0266 & 0.0030 & & -0.0259 & 0.0344 & -0.1321 & -0.0043 \\
$\sigma_{v}$ & 0.1450 & 0.0084 & & 0.2113 & 0.0904 & 0.1228 & 0.3432 \\
$\rho$ & -0.6597 & 0.0333 & & -0.3763 & 0.1448 & -0.6310 & -0.0878 \\
$\mu_{Y}$ & -2.4269 & 0.5475 & & 0.6106 & 1.0363 & -1.4616 & 2.9216 \\
$\sigma_{Y}$ & 2.1621 & 0.4693 & & 5.7855 & 3.3338 & 2.1613 & 12.7180 \\
$\lambda$ & 0.0057 & 0.0015 & & 0.0267 & 0.0183 & 0.0045 & 0.0753 \\
$\mu_{V}$ & 1.8524 & 0.4195 & & 1.8190 & 3.7166 & 0.5047 & 9.0287 \\
$\rho_{J}$ & -0.0010 & 0.0164 & & -0.0005 & 0.0111 & -0.0134 & 0.0239 \\
\hline
\end{tabular}

NOTE: The table shows the estimated parameters for the index (posterior mean and standard error) and cross-sectional summary statistics for the posterior means of the parameter estimates for the individual stocks. An overview of the stocks included in our sample and the respective estimation period can be found in the online appendix.

unconditional expectation equal to $\mu_{y}+\rho_{j} \mu_{v}$. For the index this expected value is equal to -2.68 , while for the average stock we obtain an estimate of 1.52. Again, we can clearly see that there is no such thing as the representative stock. Depending on the stock under consideration, there are both positive and negative mean jump sizes. For the remaining parameters we obtain results that are qualitatively very similar to those shown for the SV and SVJ model.

Another way of visualizing structural differences in the parameter estimates for index and single stocks is to use box-plots as given in Figure 2.A.1 and 2.A.2. The box-plots in these figures show the estimated SVJ and SVCJ jump parameters. We see in both graphs that all jump parameters exhibit a huge variation in values across stocks. In particular, however, we observe that the estimated parameters for the index (circled observation) is always located outside the inter-quartile range indicating that the index value is not representative of a typical stock.

\subsubsection{Model Choice}

We compare the models by computing Bayes factors for the models under consideration. Eraker, Johannes, and Polson (2003) showed that the odds ratio for the SV relative to the SVJ can be estimated as 
Table 2.4: Model Comparison via Bayes Factors

\begin{tabular}{lrrrr}
\hline Evidence is ... & SVJ vs. SV & SVCJ vs. SV & SVCJ vs. SVJ & SVJ vs. SVCJ \\
\hline$\ldots$ positive & 0 & 1 & 9 & 30 \\
$\ldots$ strong & 0 & 0 & 0 & 15 \\
$\ldots$ very strong & 198 & 191 & 0 & 18 \\
\hline
\end{tabular}

NOTE: This table shows the Bayes factors in a comparison of nested model specifications. The first two columns show the results of the comparison of the SVJ and the SVCJ models respectively with the SV model specification. The third column shows the results of the comparison of the SVJ and the SVCJ models. A positive value for the Bayes factor shows a preference for the model mentioned first in the column heading. We follow Kass and Raftery (1995) and interpret evidence against a model as positive if the log Odds Ratio is in between 2 and 6 , strong if it is in between 6 and 10, and very strong if it is larger than 10. An overview of the stocks included in our sample and the respective estimation period can be found in the online appendix.

$$
\frac{p(\mathrm{SV} \mid R)}{p(\mathrm{SVJ} \mid R)}=\frac{B\left(\alpha_{0}, \beta_{0}\right)}{B\left(\alpha_{0}, \beta_{0}+T\right)} \frac{1}{G} \sum_{g=1}^{G} \frac{B\left(\alpha_{0}+\sum_{t=1}^{T} J_{t}^{g}, \beta_{0}+2 T-\sum_{t=0}^{T} J_{t}^{g}\right)}{B\left(\alpha_{0}+\sum_{t=1}^{T} J_{t}^{g}, \beta_{0}+T-\sum_{t=0}^{T} J_{t}^{g}\right)}
$$

where $G$ is the number of non-discarded draws in the MCMC sample. We follow Kass and Raftery (1995) and interpret evidence against a model as positive if the log Odds Ratio is between 2 and 6 , strong if it is in between 6 and 10, and very strong if it is larger than 10 .

Table 2.4 shows that for both individual stocks and the index, the SV model is rejected when compared to the more flexible alternatives. This is a rare exception where the result from the analysis of index returns is similar to the results of single stocks analysis. The SV model specification is outperformed by the jump diffusion setup. Because of this, we no longer consider the SV model in the subsequent analysis.

Somewhat surprisingly, the SVCJ model does not consistently outperform SVJ. One reason may be that the SVJ model is not exactly nested in the SVCJ approach. The SVJ model cannot be obtained by meaningfully restricting certain parameters in the SVCJ model. The latter model is somewhat more flexible, but at the same time imposes the strong requirement that every price jump has to be accompanied by a variance jump. Since it is not clear that price and variance jumps occur in a strictly simultaneous fashion, it may be difficult for the SVCJ model to outperform the SVJ approach. 


\subsubsection{When (and why) does the Index Jump?}

\subsubsection{Simulation Results}

We conduct a simulation study to; a) demonstrate that our estimation method is able to correctly identify jump days in the empirical data, and b) determine the appropriate threshold for the posterior jump probability used to separate jump from non-jump days.

For our simulation study we simulate 1000 paths based on the SVJ and SVCJ models. We generate two simulation results for each model. First, we use the estimated parameters for the index. We then use the average parameter estimates from the single stock estimation. This ensures that we understand how sensitive the estimation procedure of posterior jump probabilities is relative to different model choices and different parameter values. We apply the Euler discretization scheme given in Equation (2.3) to simulate 4000 days per path using parameter estimates for the index and average single stocks as given in Tables 2.2 and 2.3 .

Our simulation study confirms two results that have been documented in previous papers. First, Eraker, Johannes, and Polson (2003) show that the estimation method is able to identify the model parameters. Second, Jones (2003a) shows that the Euler discretization at a daily frequency is a good approximation for the continuous time setup. A very interesting and novel result (to the best of our knowledge) is that we show how well the estimation setup is able to identify jump days.

The results of our simulation study can be found in Table 2.5. Panel A and B show the results for the SVJ and SVCJ model, respectively. On the left side we show posterior jump probabilities using the parameter values for the index, and on the right side we show the results for single stocks. We see a clear difference in the results for the posterior jump probabilities on jump and non-jump days in all four cases. The most important lesson we learn from the table is that the posterior jump probabilities for a jump day range from 0 to $100 \%$, whereas on non-jump days for both models combined we observe an upper bound for the probability of around $10 \%$ for the index and $25 \%$ for single stocks. These results clearly show that we are able to rule out false positives (identifying a jump day when there is none) when identifying jump days if we set the threshold for the posterior jump probabilities to some value higher than $10 \%$ for the index and $25 \%$ for the single stocks. However, we are unable to rule out false negatives (not identifying a jump day when there is one) when identifying jump days.

\subsubsection{Jump Day Analysis}

The issue at the core of our analysis is to study index jumps in detail. In this section we will discuss three main results; a) jumps are identified relative to the level of return 
Table 2.5: Posterior Jump Probability on Jump vs. Non-jump Days (SVCJ Model)

\begin{tabular}{|c|c|c|c|c|}
\hline \multicolumn{5}{|c|}{ Panel A - SVJ Model } \\
\hline & \multicolumn{2}{|c|}{ Index } & \multicolumn{2}{|c|}{ Single Stocks } \\
\hline & Non-jump days & Jump days & Non-jump days & Jump days \\
\hline Mean & 0.0060 & 0.3596 & 0.0311 & 0.3468 \\
\hline Median & 0.0030 & 0.0658 & 0.0194 & 0.1094 \\
\hline$Q_{99}$ & 0.0543 & 1.0000 & 0.2465 & 1.0000 \\
\hline$Q_{95}$ & 0.0164 & 1.0000 & 0.0847 & 0.9999 \\
\hline$Q_{05}$ & 0.0006 & 0.0014 & 0.0087 & 0.0127 \\
\hline$Q_{01}$ & 0.0003 & 0.0006 & 0.0061 & 0.0086 \\
\hline \multicolumn{5}{|c|}{ Panel B - SVCJ Model } \\
\hline & \multicolumn{2}{|c|}{ Index } & \multicolumn{2}{|c|}{ Single Stocks } \\
\hline & Non-jump days & Jump days & Non-jump days & Jump days \\
\hline Mean & 0.0078 & 0.3230 & 0.0266 & 0.3126 \\
\hline Median & 0.0024 & 0.1183 & 0.0155 & 0.0931 \\
\hline$Q_{99}$ & 0.0912 & 1.0000 & 0.2208 & 1.0000 \\
\hline$Q_{95}$ & 0.0268 & 0.9993 & 0.0782 & 0.9999 \\
\hline$Q_{05}$ & 0.0002 & 0.0026 & 0.0036 & 0.0099 \\
\hline$Q_{01}$ & 0.0000 & 0.0008 & 0.0017 & 0.0051 \\
\hline
\end{tabular}

NOTE: The table shows descriptive statistics for the posterior jump probability estimated by the MCMC algorithm for the SVJ model and the SVCJ model for non-jump and jump days. We perform a simulation study where 1,000 price paths are simulated based on the estimated parameters for the S\&P 100 index. In column 2 and 3 we report results based on the parameters estimated for the index, and in column 4 and 5 we report results for the parameters based on the single stocks. A day is labeled a jump day in the simulation when the draw from the uniform distribution over the interval $[0,1]$ is less than or equal to $\lambda \Delta t$, where $\lambda$ is the estimated intensity for the Poisson process driving jumps in the S\&P 100 index (see Table 2.3), and $\Delta t$ is equal to 1 day. 


\section{A JUMPING INDEX}

variance, b) we identify to distinct types of index jumps that we coin 'correlation jumps' and 'macro jumps', c) we show that the two jump types are driven by different economical environments.

The first step in this exercise is to identify the days when the index jumps. Given our simulation results, we chose to select those days as jump days where the posterior probability for a jump was greater than $10 \%$ for the SVJ model and greater than $15 \%$ for the SVCJ model. We note that these thresholds are very conservative in the sense that the probability of identifying false jump days is virtually zero. This will become important later on when we identify the two different types of index jumps.

In our sample period, we observe 31 jump days for the SVCJ model, and 28 index jump days for the SVJ model (see Tables 2.6 and 2.7). Not surprisingly, jump days exhibit mostly large negative index returns. We observe only two jump days with a positive return for the SVJ model, and one day in the case of the SVCJ model. In the following, we focus the discussion on the results of the SVJ, since; a) the SVJ model outperforms the SVCJ model more often than the other way around, and b) the results we are interested in are virtually the same for SVJ and SVCJ model. ${ }^{5}$

One surprising result from Table 2.6 is that we do not observe an increase in jump events during the financial crisis. This conclusion can be drawn from the fact that Table 2.6 shows only two days in 2008 where the index jumps. A value that is comparable to the number of jumps found in other years. However, when inspecting a list of the 20 largest index movements in Table 2.8 we see that 13 of the largest index movements actually took place in the year 2008. We would expect such an unusually high number of large daily movements to trigger a large number of jump days. When using the $10 \%$ threshold, the SVJ model identifies two jump days for 2008 (as seen in Table 2.6), while the SVCJ model identifies three jump days in 2008 using the $15 \%$ threshold.

Table 2.8 provides intuition on why we do not see a large number of jump occurrences coinciding with the unusually large index returns. Since both models show similar results, we limit the following discussion to columns 3-5 of the table, which show the results for the SVJ model. As can be seen from the Table 2.8, most days with large movements and a low posterior jump probability also have a large value for the variance. For example, the movements of about $-6 \%$ on October 7 and November 19 of 2008, are within the bounds induced by two standard deviations of the volatility value for these days. This means that a pure stochastic volatility model would be able to generate these movements. This explanation is not applicable to all our observations. For example in Table 2.8, we observe

\footnotetext{
${ }^{5}$ The tables containing the results for the SVCJ can be found in the online appendix.
} 


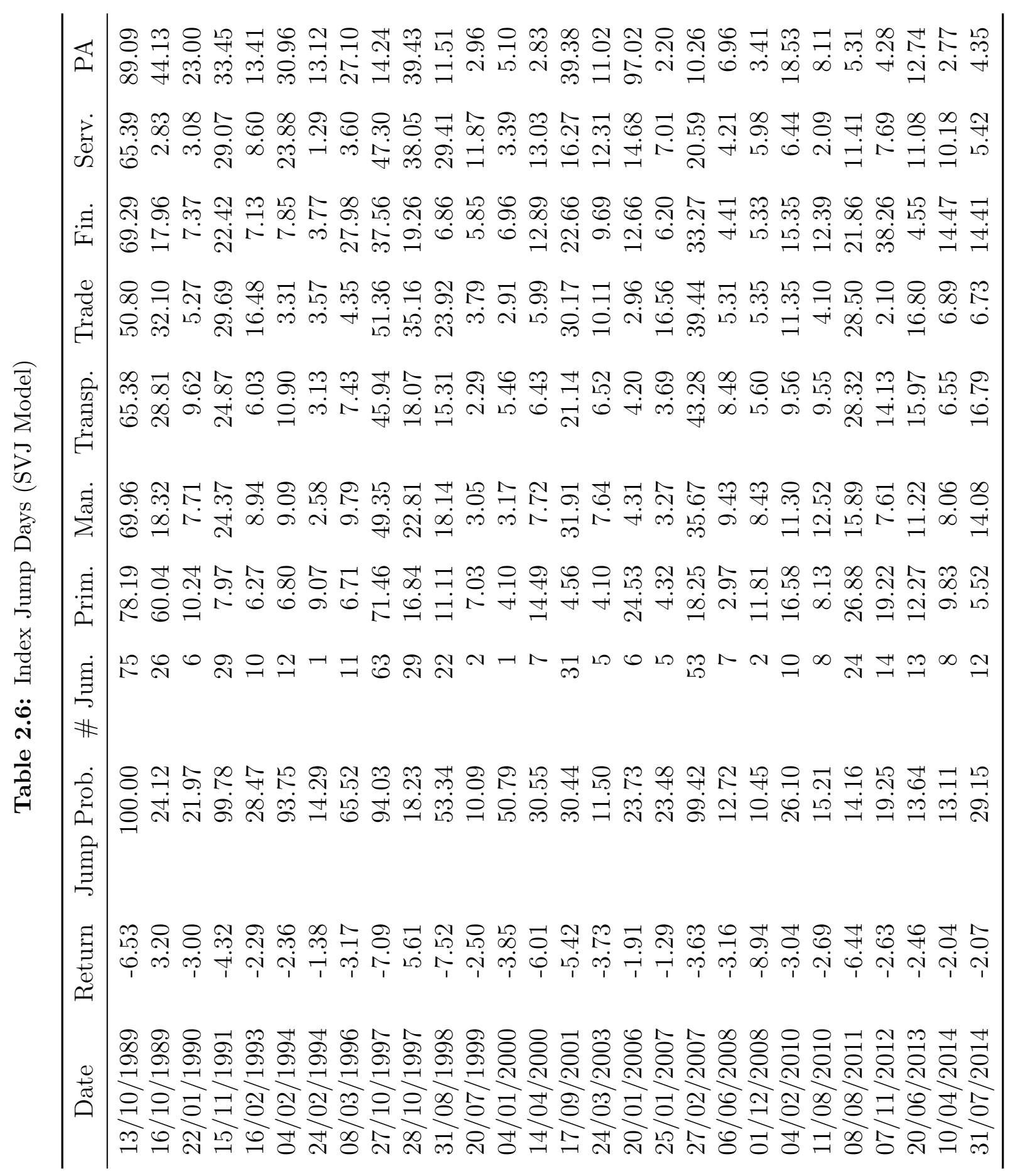

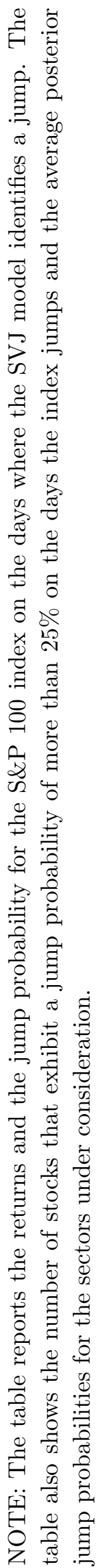




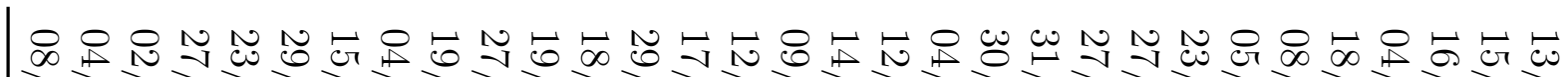

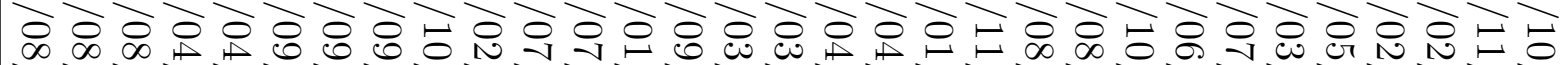

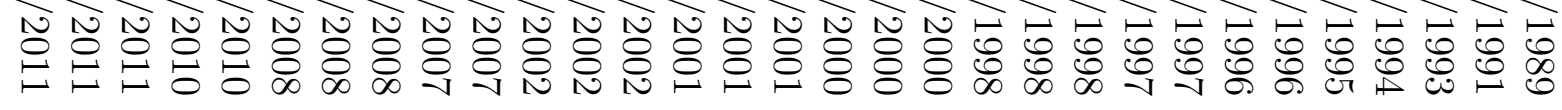

فُ

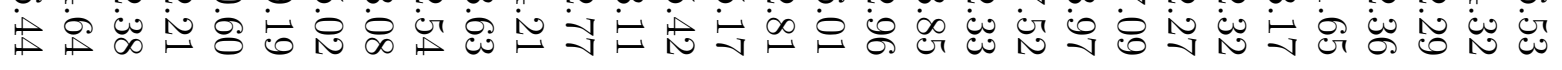

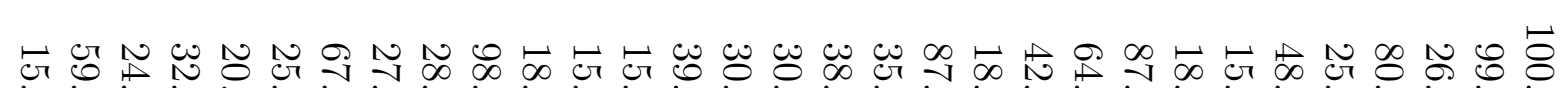

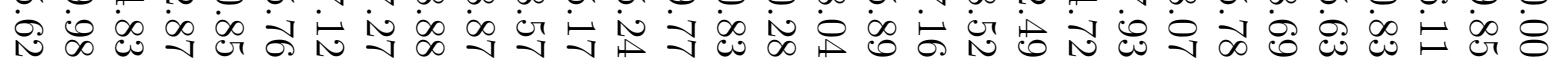

ن্ ํㅓㅂ

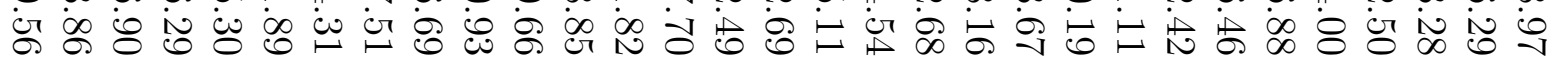
D N

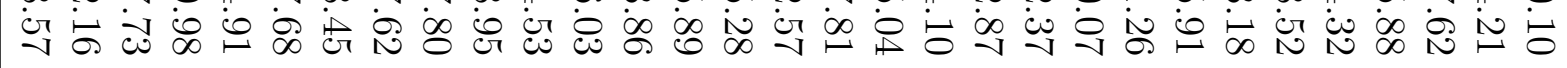

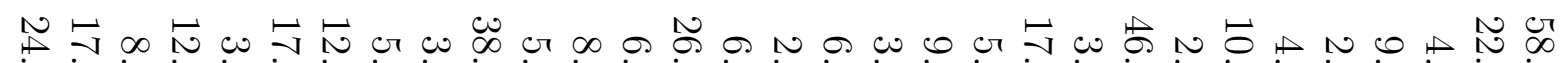

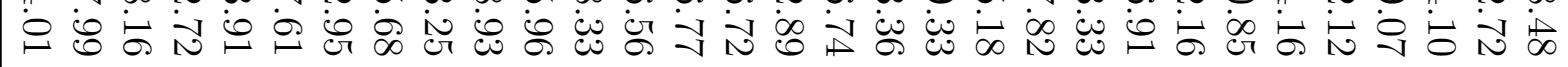

Uึ

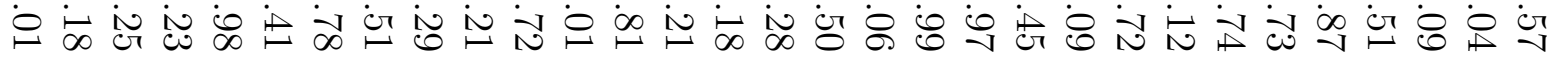

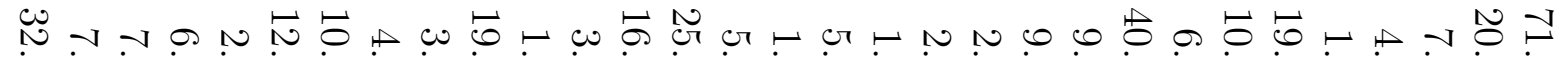

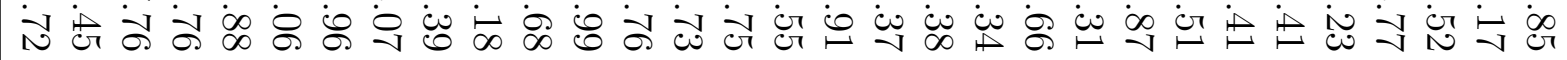

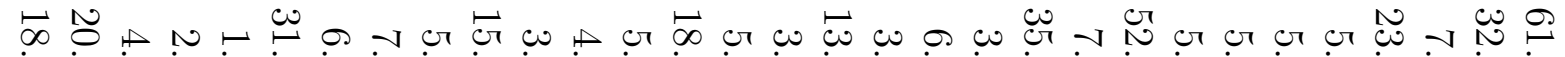
$\dot{\infty}$ 궈 ๑!

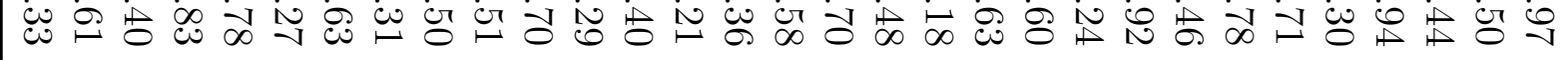


Table 2.8: Posterior Jump Probabilities and Diffusive Bounds

\begin{tabular}{|c|c|c|c|c|c|c|c|}
\hline \multirow[b]{2}{*}{ Date } & \multirow[b]{2}{*}{ Return } & \multicolumn{3}{|c|}{ SVJ } & \multicolumn{3}{|c|}{ SVCJ } \\
\hline & & Prob. & UB & LB & Prob. & UB & LB \\
\hline 13/10/1989 & -6.5273 & 1.0000 & 1.7596 & -1.6947 & 1.0000 & 1.3308 & -1.2577 \\
\hline $27 / 10 / 1997$ & -7.0927 & 0.9403 & 2.9335 & -2.8686 & 0.8793 & 2.7582 & -2.6851 \\
\hline $31 / 08 / 1998$ & -7.5165 & 0.5334 & 3.8614 & -3.7965 & 0.4249 & 4.3498 & -4.2767 \\
\hline $14 / 04 / 2000$ & -6.0088 & 0.3055 & 3.5328 & -3.4678 & 0.3804 & 3.7395 & -3.6664 \\
\hline $29 / 09 / 2008$ & -9.1862 & 0.0649 & 5.5909 & -5.5259 & 0.2576 & 7.2023 & -7.1292 \\
\hline $07 / 10 / 2008$ & -5.9769 & 0.0087 & 6.2020 & -6.1370 & 0.0856 & 7.9580 & -7.8850 \\
\hline $09 / 10 / 2008$ & -7.9154 & 0.0156 & 6.5012 & -6.4362 & 0.1447 & 8.1528 & -8.0797 \\
\hline $13 / 10 / 2008$ & 10.6551 & 0.0158 & 6.8262 & -6.7613 & 0.0073 & 8.4501 & -8.3771 \\
\hline $15 / 10 / 2008$ & -8.7550 & 0.0291 & 6.5080 & -6.4431 & 0.0428 & 8.0643 & -7.9912 \\
\hline $22 / 10 / 2008$ & -5.9697 & 0.0103 & 6.5767 & -6.5117 & 0.0140 & 7.8162 & -7.7432 \\
\hline $28 / 10 / 2008$ & 10.2961 & 0.0098 & 6.8499 & -6.7850 & 0.0079 & 7.7964 & -7.7233 \\
\hline $13 / 11 / 2008$ & 6.3306 & 0.0039 & 6.5990 & -6.5340 & 0.0134 & 7.0829 & -7.0098 \\
\hline $19 / 11 / 2008$ & -6.0350 & 0.0126 & 6.4398 & -6.3749 & 0.0257 & 6.8154 & -6.7423 \\
\hline $20 / 11 / 2008$ & -6.6214 & 0.0160 & 6.6009 & -6.5360 & 0.0248 & 6.9304 & -6.8574 \\
\hline $21 / 11 / 2008$ & 6.0205 & 0.0034 & 6.7677 & -6.7027 & 0.0023 & 7.0510 & -6.9779 \\
\hline $24 / 11 / 2008$ & 5.8915 & 0.0036 & 6.5296 & -6.4646 & 0.0021 & 6.8025 & -6.7294 \\
\hline $01 / 12 / 2008$ & -8.9448 & 0.1045 & 5.9946 & -5.9296 & 0.0637 & 6.2029 & -6.1298 \\
\hline $10 / 03 / 2009$ & 6.0499 & 0.0056 & 5.3532 & -5.2882 & 0.0079 & 5.4093 & -5.3362 \\
\hline $23 / 03 / 2009$ & 6.7381 & 0.0156 & 4.6809 & -4.6159 & 0.0052 & 4.7052 & -4.6321 \\
\hline 08/08/2011 & -6.4430 & 0.1416 & 3.8892 & -3.8242 & 0.1562 & 5.0169 & -4.9438 \\
\hline
\end{tabular}

NOTE: This table shows the 20 largest daily returns observed for the S\&P 100 index. We show the return, the posterior jump probability of the index, the upper and lower bound for the diffusive part of the model computed as $\mu \pm 2 \sqrt{V_{t-1}}$ for both jump models.

large positive movements on October 13 and 28 of 2008. On both days the index gained more than $10 \%$, a movement outside of the bounds induced by the diffusive component, but no jump was identified on both days. The reason for this can be explained by the fact that we are not able to rule out false negatives when identifying jump days. As discussed, our estimation strategy is able to rule out false positives. It is the case, therefore, that days when the return observation is outside the diffusive bounds and are not identified as jumps can be considered as false negatives.

The main takeaway from Table 2.8 is that given our model framework jump days are not identified only by large index movements. The index movement has to be seen relative to the underlying value of the variance process. If the variance for a particular day is large the diffusive component of the return process can generate a high index return. As shown in both tables, the diffusive component is even able to capture a number of the 20 highest returns in absolute value in our sample. However, if the variance is small a relatively 


\section{A JUMPING INDEX}

modest index movement can be identified as a jump event since we would need a jump component on top of the diffusive part to generate such an observation.

The next question arising naturally is how individual stocks behave on these index jump days. A prior would certainly be that many individual stocks also exhibit jumps on the same days the index jumps. We identify jump events for single stocks by setting the posterior jump probability to $25 \%$, as indicated by our simulation study, with the parameter values set to the average value for the stocks. Unexpectedly, our results show that we find days when the index jumps, but only a very small number of stocks jump also. For example, in Table 2.6 we see that on January 4, 2000, only one stock jumps on the day where the index exhibits a posterior jump probability of over $50 \%$, which constitutes a very high value as demonstrated by our simulation exercise. On the other hand, we observe 75 stocks jumping on October 13, 1989, when we have a posterior jump probability of $100 \%$ for the index.

To understand why there are such different jump patterns, we list the 5 jump days with the largest and lowest number of stocks jumping for the SVJ model in Table 2.9.

In Panel A, we observe days where only one to five stocks jump despite the fact that the posterior jump probability for the index is higher than $10 \%$ for all five days. What does this table imply in terms of how we interpret the occurrence of an index jump for these days? Obviously, given the small number of stocks jumping simultaneously with the index, there is no additivity in the strict sense that an index jump is the sum of jumps in individual stock prices. On the other hand, the index return has to be equal to the weighted sum of individual stock returns. The hypothesis therefore is that, to a very large extent, index jumps are generated by diffusive price movements in the individual stocks, which happen to move mostly in the same direction.

This reasoning can be verified by the results in Table 2.10, which show that in all of the five days in Panel A the overwhelming majority of stocks exhibit a returns on index jump days that moves into the same direction. So why is it that the individual stocks do not also jump on these days? A look at the average conditional variance of stock returns around index jump days confirms the intuition that individual stock return volatility is much higher than index volatility, so that it is more likely for stocks to have large returns in absolute value generated by just the diffusive component of the stochastic process.

In general it holds that the index return is given by the weighted average of the returns of the single stocks. On 'normal' days diversification would result in an index return which is small enough in absolute value not to be considered an index jump. Put differently, due to diversification the volatility of the index is lower than the weighted 


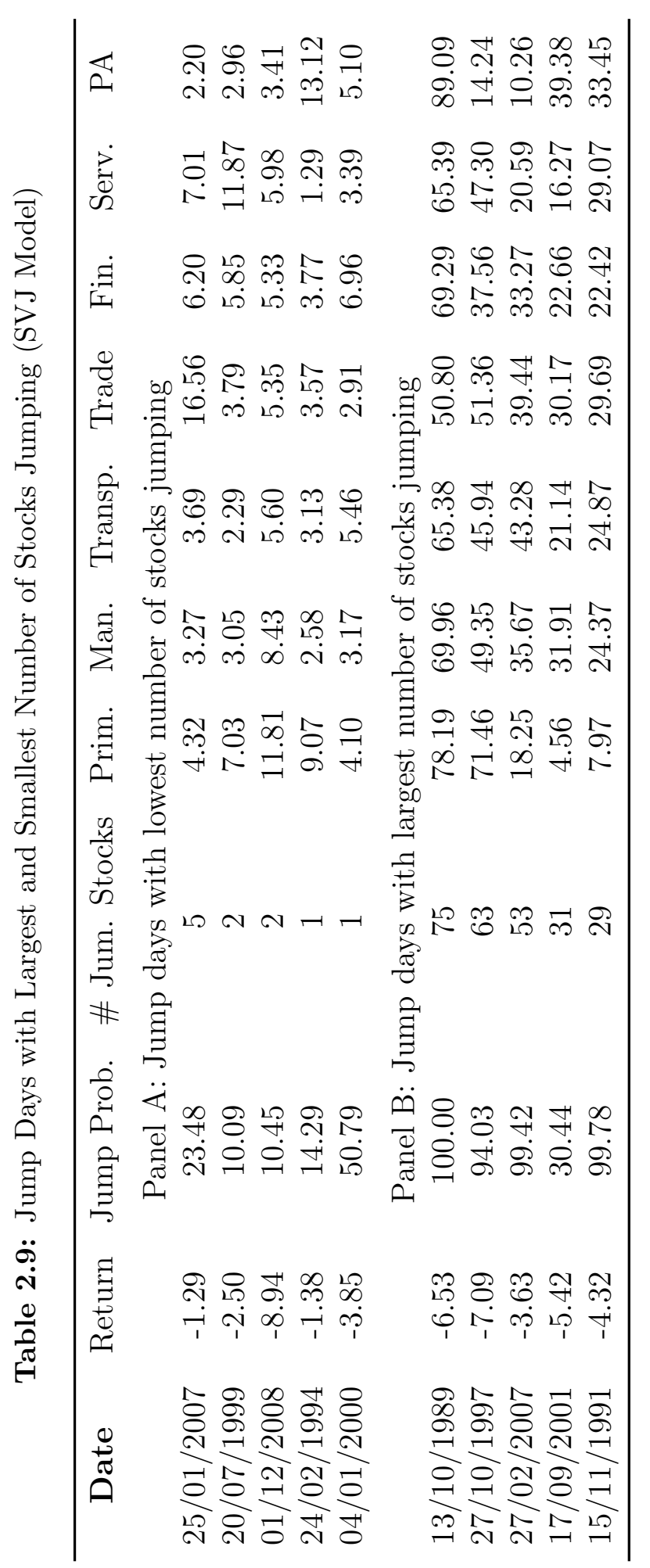

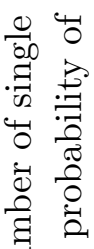
寻苜

苗 费

获 离

秀榙

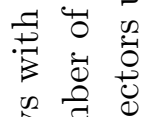

穵 节

20 5

幽

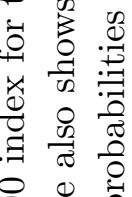

$8 \stackrel{0}{0} \stackrel{0}{0}$

这索焉

击 $\dot{\overrightarrow{0}}$

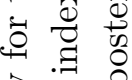

굴 0

के

है, 吾

잌.

ఠ

靑

สิ .․․

鸷害

$=0$

击节索

刍

원

迹

需

氖䓫

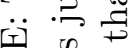

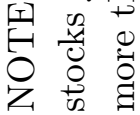


Table 2.10: Signs of Individual Stock Returns on and around Index Jump Days (SVJ Model)

\begin{tabular}{crrrr}
\hline Date & $\# R>0$ & $\% R>0$ & $\# R<0$ & $\% R<0$ \\
\hline \multicolumn{5}{c}{ Panel A: Lowest number of stocks jumping } \\
$25 / 01 / 2007$ & 12.00 & 0.13 & 81.00 & 0.87 \\
$20 / 07 / 1999$ & 19.00 & 0.20 & 75.00 & 0.80 \\
$01 / 12 / 2008$ & 0.00 & 0.00 & 93.00 & 1.00 \\
$24 / 02 / 1994$ & 13.00 & 0.14 & 77.00 & 0.86 \\
$04 / 01 / 2000$ & 12.00 & 0.13 & 83.00 & 0.87 \\
Panel B: Largest number of stocks jumping \\
$13 / 10 / 1989$ & 0.00 & 0.00 & 92.00 & 1.00 \\
$27 / 10 / 1997$ & 0.00 & 0.00 & 96.00 & 1.00 \\
$27 / 02 / 2007$ & 0.00 & 0.00 & 93.00 & 1.00 \\
$17 / 09 / 2001$ & 14.00 & 0.14 & 85.00 & 0.86 \\
$15 / 11 / 1991$ & 2.00 & 0.02 & 90.00 & 0.98 \\
\hline
\end{tabular}

NOTE: Panel A of the table shows the five days where an index jump is accompanied by the lowest number of single stock jumps. Panel B of the table shows the five days where an index jump is accompanied by the highest number of single stock jumps. Column 2 and 4 show the number of positive and negative stock returns, respectively. Column 3 and 5 show the same information as percentages.

average of the volatilities of the single stocks when the correlation between the index constituents is below one. However, when the correlation between the individual stocks goes up significantly stock returns will basically all have the same sign (here: negative) destroying the diversification effect. Compared to the direct history of index returns this results in a relatively large and negative index return. Conditional on the index parameters and a much lower level of index volatility in the days before the correlation jumps up such large movements constitute a nearly impossible event for a pure stochastic volatility model and therefore cannot be explained by the diffusive component of the index alone. Hence, a large negative return of the index then 'has to be' identified by the model as a jump, since the model predicted volatility of a day where the correlation between the constituents jumps up did not anticipate the unpredictable large increase in stock correlation. I.e., jumps are induced by a sudden increase in single stock correlation.

However, correlation does not give the full picture. To find out if all the index jumps in our sample are likely to be the result of a correlation moving (or jumping) towards one, we analyze the jump probabilities of the different industries our sample firms belong to. ${ }^{6}$

\footnotetext{
${ }^{6}$ The companies are divided in industries according to the first two digits of the SIC codes that can be found at siccode.com. The sector that we label Primary groups all the companies belonging to the SIC codes corresponding to Agriculture, Forestry, Fishing and Mining. In the sector labeled Manufacturing we group companies that have SIC codes corresponding to Manufacturing and Construction. Companies
} 
Again, for the sake of brevity we concentrate our following analysis on the results found for the SVJ model and the threshold for the jumps in the single stocks is set to $25 \%$ posterior jump probability. Panel B of Table 2.9 shows the five days with the largest number of index constituents jumping when the index jumps. The table also contains information on the average posterior jump probabilities across industry sectors. Here we see a clear difference between the index jump days listed in both panels. For Panel B we observe an average jump probability across the sectors that are significantly higher than the ones observed for the days in Panel A. We see that the index jump is accompanied by jumps in constituents of all sectors on these days. This is a clear distinction to the occurrences of the days listed in Panel A, where we have significant average jump probabilities of at most one sector on these days. The important conclusion we draw from this difference is that the index jumps listed in Panel A are generated from diffusive movements in the stocks that go largely into the same direction. We term these jumps 'correlation jumps'. Whereas the jumps in Panel $\mathrm{B}$ are generated by jump movements in the constituents across all sectors. We term these jump events 'macro-driven jumps'.

At this point, it is instructive to briefly come back to our discussion on the identification of jump days. Since our estimation method rules out false positives with a probability close to one, we are very confident that the 'correlation jumps' we identify are indeed jump events induced by the models. This is important, since one of the central contributions of our analysis is the identification of two economically distinct jump events, and we have to make sure that the identification of these jump days does not occur merely because of a false rejection of a hypothesis.

To analyze our distinction of the differences in the jump days in more detail we turn to Figure 2.A.3, which shows the sector jump probabilities for the five days with the largest number of stocks jumping in the top panel and the five days with the lowest number of stocks jumping in the bottom panel. We find big differences in the overall level of sector jump probabilities between both panels. It can easily be seen that the sector averages in the upper panel of Figure 2.A.3 are much higher than the averages in the lower panel. We can also see that for every day in the upper panel at least six of the seven sectors exhibit a posterior jump probability above 10\% (see also Table 2.9). This implies that the jump in the index on the days depicted in the upper panel of Figure 2.A.3 is accompanied by jumps in single stocks of at least six out of seven industry sectors. This stands in clear contrast to the jump days depicted in the lower panel of the same figure. Here at most

related to Transport and Public utilities are included in the third sector labeled Transport. The sector that we label Trade is comprised of the companies under the SIC codes of Wholesale Trade and Retail Trade. The sector we call Finance is constituted by the companies that relate to the siccode category called Finance, Insurance and Real Estate. We label the second to last sector Services as it is also labeled on the SIC webpage. For sake of convenience we call the last sector $P A$, a short version of Public Administration, as on the SIC webpage. 


\section{A JUMPING INDEX}

one sector has an average jump probability larger than 10\%, implying that the index movement is not accompanied by unusually large movements in the sectors.

It is interesting to note that a quick Google search for the days depicted in the upper panel of Figure 2.A.3 gives back stories of stock market crashes as first or second hit. For example, the jumps on October 13, 1989 and October 27, 1997 can be linked to crucial market wide events. The first event was termed a "mini-crash" relating to a drop in prices in the junk bond markets, whereas the second relates to an economic crisis in Asia (sometimes called the 'Asian flu'). These two days also represent the two largest percentage drops in the index for our sample. Also, stock market reports for February 27, 2007, frequently mention a large drop in the Chinese stock market as an important reason for the big loss in the $\mathrm{S} \& \mathrm{P} 100$ on that day, so the reasons for this jump are similar to the ones described above. In summary, the reason why we refer to these jumps as 'macro-driven jumps' is due to the fact that many industries jump on these days, and the reasons can be traced back to macroeconomic events.

On the other hand, the jump days depicted in the lower panel of Figure 2.A.3 are characterized by very small sector jump probabilities. The fact that we identify an index jump at all is more due to the tendency of the statistical properties of the stochastic process for stock prices to label a price move as a jump mostly when it is too large to be generated with a sufficiently high probability by the diffusive component. Because these jumps are driven mostly by the correlation effect described above, we refer to them as 'correlation jumps'.

\subsection{Conclusions}

This paper analyzes stochastic equity price models for a large cross section of individual stocks. Specifically, 201 stocks that were included in the S\&P 100 index at some time in the period we analyze. Furthermore, we compare the estimation results for the individual stocks to the results for the S\&P 100 index. The models under consideration allowed for a jump component in the return, as well as in the variance process. The estimation method employs a Bayesian econometric framework using a MCMC algorithm to compute the moments of the posterior distribution.

Our results show that the parameters governing the stochastic processes for individual stocks are very heterogeneous. Unsurprisingly, the volatility process for individual stocks is much higher than the volatility process for the index. Furthermore, we find a less pronounced leverage effect in the individual stocks compared to the index. Considering the distribution of the jumps in returns we can show that the stylized fact of negative average jump size found in the analysis of indices does not carry over to individual stocks. 
Some stock exhibit negative mean jump sizes where others have positive mean jump sizes. A novel result of our study is that we show, via a simulation study, that our estimation is able to cleanly identify jump days versus non-jump days.

We find a surprising result when we look at the behavior of the index's individual stocks on index jump days. We identify two types of index jumps. The first jump type occurs when many stocks exhibit a diffusive movement in the same direction (usually negative return movement). Therefore, we call this jump type 'correlation jumps'. The second index jump type is generated by jumps in a large number of stocks across all sectors of the economy, which is why we name those jumps 'macro-drive jumps'. Surprisingly, we find that the models under consideration do not identify an unusually large number of jumps during 2008 financial crisis. Intuitively, this can be explain by the prolonged high levels of volatility during that time which implies that large index returns can be generated by a pure stochastic volatility model. 


\section{Appendix}
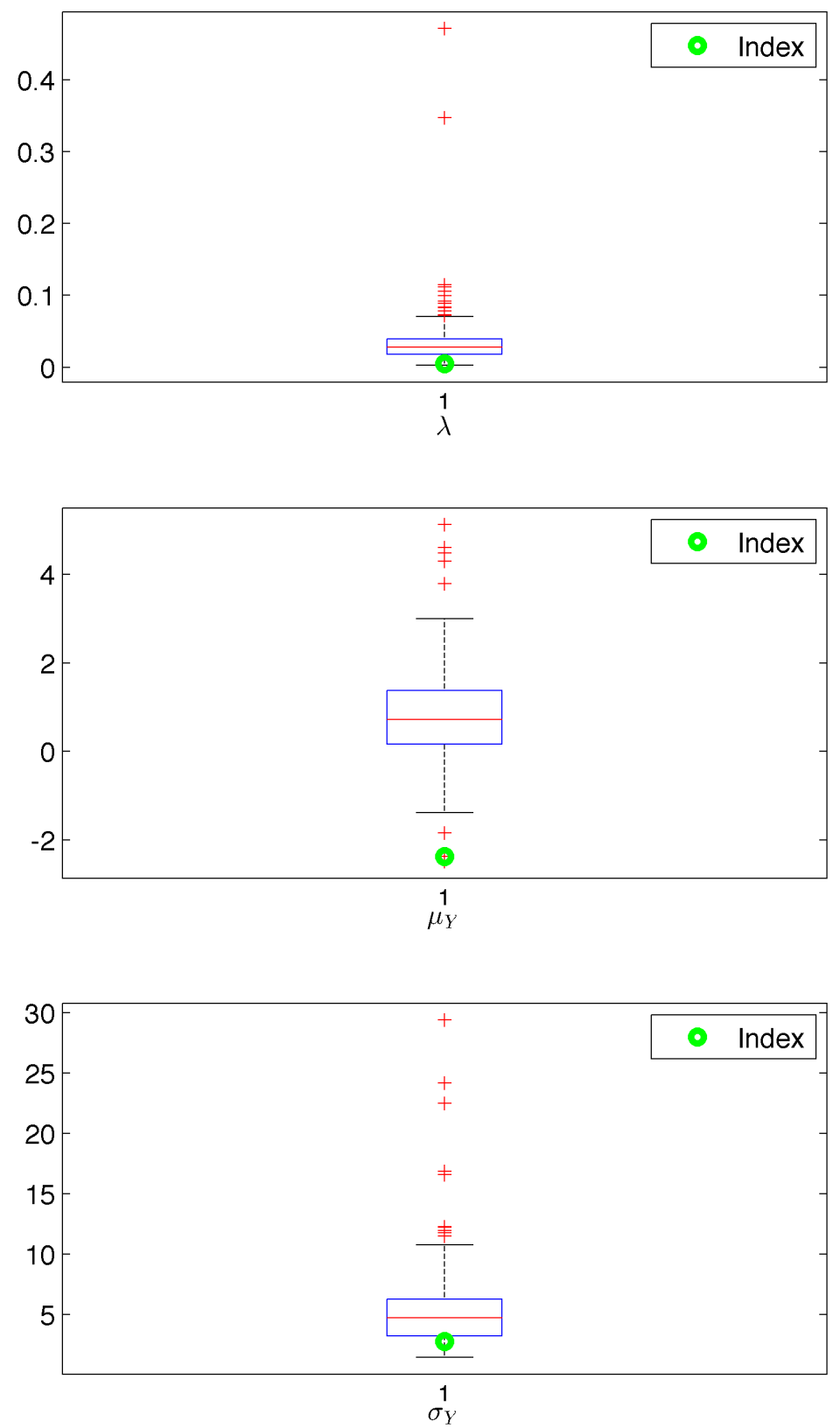

Figure 2.A.1: Box Plots for Parameters of SVJ Model

This figure shows the box plots for the parameters characterizing the SVJ model. The top box plot shows the estimates of jump intensity $\lambda$ for single stocks and the index in green. The middle box plot shows the estimates for the expected jump size in return $\mu_{Y}$ for single stocks and the index. The bottom box plot shows the estimates for the standard deviation of the jump size in return $\sigma_{Y}$. 

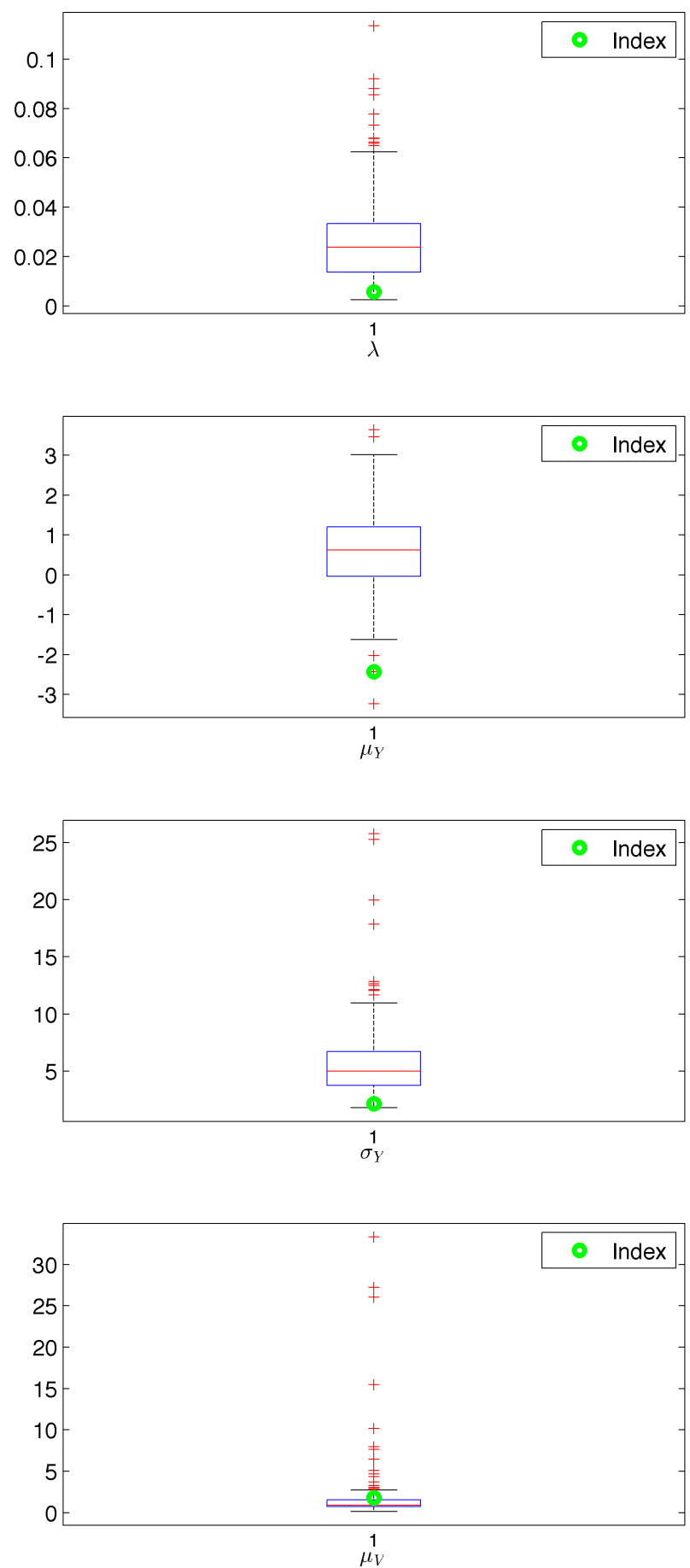

Figure 2.A.2: Box Plots for Parameters of SVCJ Model

This figure shows the box plots for the parameters characterizing the SVCJ model. The top box plot shows the estimates of jump intensity $\lambda$ for single stocks and the index in green. The second box plot shows the estimates for the expected jump size in return $\mu_{Y}$ for single stocks and the index. The third box plot shows the estimates for the standard deviation of the jump size in return $\sigma_{Y}$. The bottom box plot shows the estimates for the expected jump sizes in variance $\mu_{V}$. 

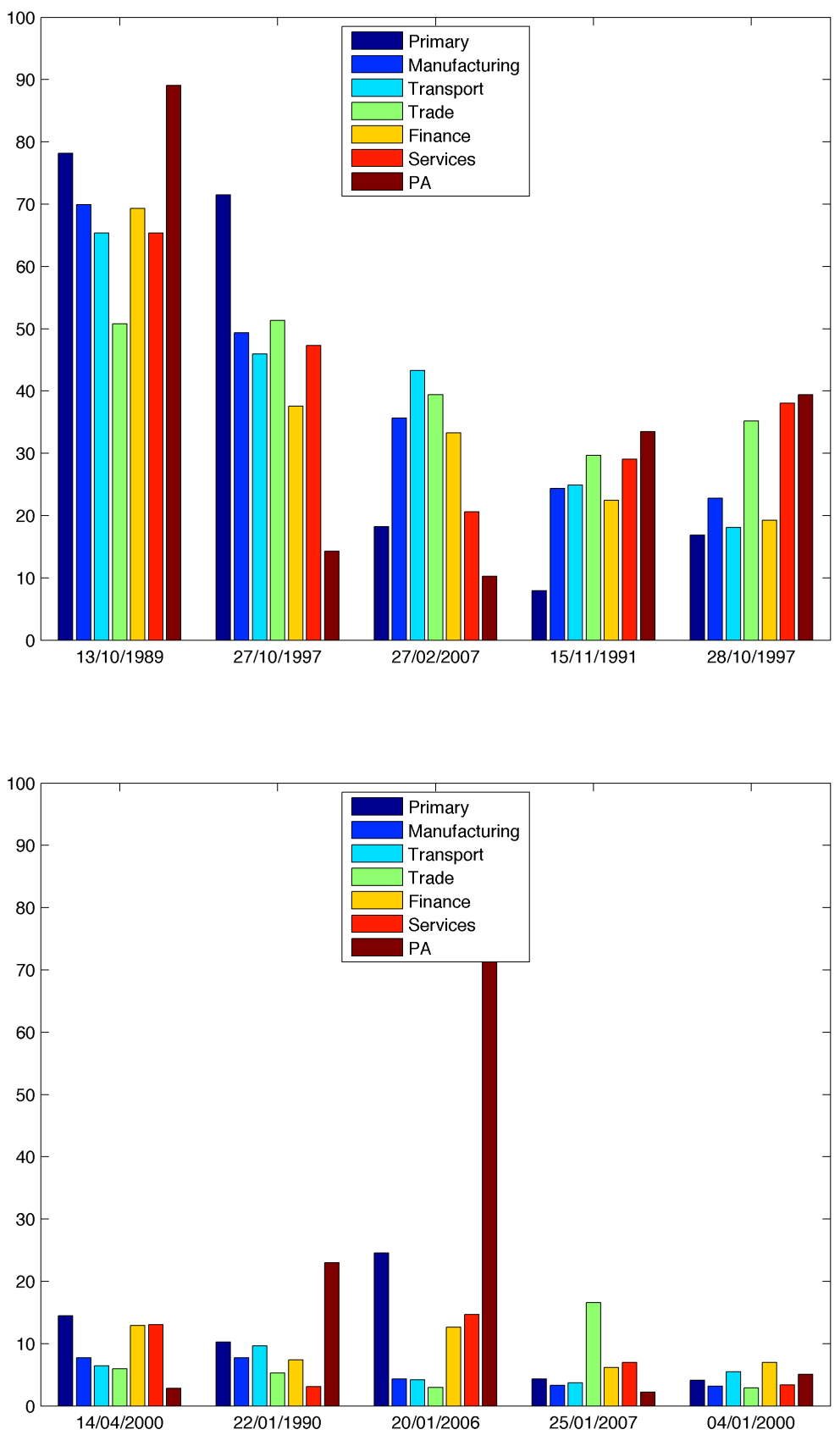

Figure 2.A.3: Industry Jump Probabilities on Index Jump Days (SVJ Model)

This figure shows the average posterior jump probabilities for the different sectors on the jump days identified by the SVJ model. The upper graph shows the five days where an index jump is accompanied by the highest number of single stock jumps and the lower graph shows the five days where an index jump is accompanied by the lowest number of single stock jumps. 


\section{Chapter 3}

\section{Tailoring the Errors*}

\subsection{Introduction}

Effective risk management is the foundation for a solid financial system. Therefore, measuring and managing risk is of utmost importance for financial institutions such as banks, pension funds and insurance companies. Supervisors and regulators demand these institutions to provide assessments of the riskiness of their portfolios. This quantification of risk takes the form of tail risk measures, which gauge the potential loss of the portfolio under extremely unfavorable market conditions.

Among the many existing approaches to compute risk measures, the most common one is based on estimating the volatility of the portfolio and then use it to get a measure of the portfolio tail risk. ${ }^{2}$ Although many years have passed since its introduction in the financial econometrics literature, the GARCH model from Bollerslev (1986) is still very popular among practitioners to compute tail risk measures, yet the growing availability of High Frequency data resulted in a superior measurement of volatility and also led to a better understanding of returns' properties as Maheu and McCurdy (2011) point out.

Our approach to tail risk measures starts from an observation in Andersen, Bollerslev, Diebold, and Labys (2003), who find that returns are approximately normally distributed when standardized with current Realized Volatility and obtain VaR with a Mixture model where Realized Volatility is linked to the mixing distribution. Therefore, our scope is to find a mixing distribution, which results in accurate daily VaR forecasts.

As our interest is in daily VaR forecasts we also require a time series model to capture the salient features of Realized Volatility and therefore adopt the HAR model of Corsi (2009) for the expected volatility and let the stochastic component to be drawn from a parametric distribution, which characterize the mixing distribution.

\footnotetext{
${ }^{*}$ This chapter is based on joint work with Peter Schotman and Stefan Straetmans.

${ }^{2}$ For an overview of the different methods to compute tail risk measures we refer to Kuester, Mittnik, and Paolella (2006) and Nieto and Ruiz (2016).
} 
Our first model is a multiplicative component one where the volatility shocks are driven by an inverse Gamma $(i G)$ distribution. This specification slightly resembles the model in Engle and Gallo (2006), where volatility shocks are drawn from a Gamma distribution. However, our specification allows to obtain a closed form density of returns in form of a Student $-t$, with degrees of freedom inherited by the volatility process.

Our second model stems from the findings in Andersen, Bollerslev, Diebold, and Labys (2001), who empirically show that a log-normal density fits reasonably well the unconditional distribution of volatility. Therefore, our second stochastic volatility model for log Realized Variance is characterized by normally distributed shocks with constant Volatilityof-Volatility. Assuming normality of volatility shocks might be restricting and therefore as a third model, we depart from this assumption by allowing the volatility shocks to be drawn from of a Student $-t$ distribution. An alternative reason for which volatility is not log-normal is due to time-varying Volatility-of-Volatility. Vol-of-Vol measures how uncertain we are about tomorrow's volatility and therefore the VaR forecast would be highly impacted if the Vol-of-Vol exhibits strong time variation. Therefore, our fourth model encompasses this feature as in the model introduced by Corsi, Mittnik, Pigorsch, and Pigorsch (2008), where Vol-of-Vol is driven by a GARCH model.

For risk management purposes, we might not only be interested in the left tail of the return distribution. This is the case when an investor holds a short position for a given security. In such a situation the tail risk derives from an extreme upward movement of the security the investor holds. An empirical feature of the return distribution is that it exhibits left skewness, implying that a negative return is more likely to occur than a positive one: this stylized fact is also known as leverage effect. In a stochastic volatility framework this feature has been introduced by Taylor (1982) and further investigated by $\mathrm{Yu}$ (2005). Our fifth model directly follows from these empirical findings and enriches the log-normal specification by allowing for a contemporaneous effect between returns and volatility shocks.

For these five time series specifications for volatility we obtain the return distribution and from it we compute Value-at-Risk. We assess the usefulness of these models in delivering appropriate VaR forecast according to different backtesting criteria. For long-positions, results show that the best performing models are the time-varying Vol-of-Vol model and the Log-Normal model, with the inverted Gamma specification always resulting in a too conservative estimate of VaR and the $\log -t$ model highly resembling the Log-Normal model due to the high estimates of the degrees of freedom of volatility shocks. On the other hand, when considering short positions we find that the model with leverage is the best performing model.

The chapter is organized as follows: section two introduces the methodology and the time 
series models, section three shows the results and the implications of the different time series specifications and section four concludes the paper.

\subsection{Methodology}

We assume that the log-price process evolves according to the continuous diffusion:

$$
d p_{s}=\beta_{s} d s+c_{s} d W_{s}
$$

where $W_{s}$ is a standard Brownian motion and $\beta_{s}$ and $c_{s}$ denote the drift and the instantaneous volatility processes independent of $W_{s}$. The daily return is obtained as the integral of (3.1) over the current day

$$
r_{t+1}=\int_{t}^{t+1} \beta_{s} d s+\int_{t}^{t+1} c_{s} d W_{s}
$$

The return process is conditionally normal centered in zero and with Integrated Variance

$$
\sigma_{t+1}^{2}=\int_{t}^{t+1} c_{s}^{2} d s
$$

The Integrated Variance cannot be directly observed and hence must be estimated. A consistent estimator for it is given by the Realized Variance, the sum of intraday squared returns. Andersen, Bollerslev, Diebold, and Ebens (2001) is one of the first studies to analyze the properties of Realized Variance for equity data. Their empirical results show that returns are approximately normally distributed when standardized by Realized Volatility. To obtain a leptokurtik density of returns we adopt the Mixture model introduced by Andersen, Bollerslev, Diebold, and Labys (2003). More formally, the predictive density of returns given the current information set, $I_{t}$ is given by:

$$
p\left(r_{t+1} \mid I_{t}\right)=\int f\left(r_{t+1} \mid \sigma_{t+1}^{2}\right) g\left(\sigma_{t+1}^{2} \mid I_{t}\right) d \sigma_{t+1}^{2}
$$

where $p(\cdot)$ is the predictive density of the return, $f(\cdot)$ is the normal density and $g(\cdot)$ is the density of Realized Variance. The distribution of Realized Variance is therefore the element that determines the predictive density of returns. As such, in the following section we introduce several competing models in order to capture the salient features of volatility. 


\subsubsection{Volatility Models}

The first discrete time model we consider is a multiplicative one:

$$
\sigma_{t+1}^{2}=\mu_{t+1} \eta_{t+1}
$$

where $\eta_{t+1}$ is a stochastic component drawn from an Inverted Gamma distribution, $i G\left(\frac{\nu}{2}, \frac{s}{2}\right)$, where $\nu$ determines the shape and $s$ the scale. We further restrict $s=\nu-2$ to impose that the conditional expectation of $\sigma_{t+1}^{2}$ to be equal to $\mu_{t+1}$, that is known at time t. Using this dynamic specification for $\sigma_{t+1}^{2}$ the predictive density of return has a close form given by a Student-t with degrees of freedom directly inherited from the inverted Gamma process in (3.5), more precisely

$$
r_{t+1} \mid I_{t} \sim t\left(0, \lambda_{t+1}, \nu\right)
$$

where $\lambda_{t+1}=\sqrt{\frac{\nu-2}{\nu} \mu_{t+1}}$ controls the scale and $\nu$ the shape of the return distribution: this implies that the scale of the return distribution changes with current market condition and it is weighted according to the degrees of freedom parameter, $\nu$. Given that, the return distribution inherits the properties of the variance process we denote this specification $i G$ from now on.

Estimation of model (3.5) is performed in two steps by first separating the predictable component of volatility, $\mu_{t+1}$, from the unexpected one, $\eta_{t+1}$ and in a second step by fitting an inverted Gamma via Maximum Likelihood. To filter out the predictable component $\mu_{t+1}$, we fit the classical HAR model introduced by Corsi (2009) and then we set equal to the fitted values. More precisely, the first step sets the conditional mean $\mu_{t+1}$ equal to the HAR structure

$$
\mu_{t+1}=\alpha_{0}+\alpha_{1} \sigma_{t}^{2}+\alpha_{2} \sigma_{t}^{2, w}+\alpha_{3} \sigma_{t}^{2, m}
$$

where $\sigma_{t}^{2, w}$ is the average Realized Variance over the previous five days and $\sigma_{t}^{2, m}$ is obtained in the same fashion but over twenty one days. A characteristic of this specification is that the multiplicative structure allows for an heteroskedastic behaviour of $\sigma_{t+1}^{2}$. In fact, the conditional variance of $\sigma_{t+1}^{2}$ is given by:

$$
\mathbb{V}_{t}\left[\sigma_{t+1}^{2}\right]=\frac{2}{\nu-4} \mu_{t+1}^{2}
$$


which only exists if $\nu>4$.

We write the second specification by modeling the log of Realized Variance and from now on we will refer to this specification as Lognormal or $L N$ in short.

$$
\log \sigma_{t+1}^{2}=m_{t+1}+\omega \epsilon_{t+1}
$$

where $m_{t+1}$ is the conditional expectation of $h_{t+1}$. This model stems from the results in Andersen, Bollerslev, Diebold, and Labys (2001), who find that Realized Variance is lognormally distributed and therefore $\epsilon_{t+1}$ is standard normally distributed. The remaining parameter is $\omega$, which captures in this specification the constant volatility of volatility. However the conditional variance of $\sigma_{t+1}^{2}$ is not constant and follows from simple statistical arguments regarding the variance of a log-normal random variable with parameters $m_{t}$ and $\omega$. In fact, under this specification the conditional mean and variance of $\sigma_{t+1}^{2}$ are given by

$$
\mathbb{E}_{t}\left[\sigma_{t+1}^{2}\right]=e^{m_{t}+\frac{1}{2} \omega^{2}} \quad \mathbb{V}_{t}\left[\sigma_{t+1}^{2}\right]=\left(e^{\omega^{2}}-1\right) e^{2 m_{t}+\omega^{2}}
$$

As we have done for the multiplicative specification we also assume that $m_{t}$ has a HAR specification.

Our third specification introduces the so-called leverage effect, which is the empirical feature of the data where negative returns are associated with high volatility values. In order to incorporate this effect in our framework, we consider the $L N$ specification and enrich it with the correlation, $\ell$, between shocks to volatility and returns. In the subsequent sections we will denote this model Lev to distinguish it from the others. Furthermore, for the details on how to introduce leverage in the Mixture model defined in (3.4) we refer to the appendix.

The third model directly follows from (3.9) but differs from it because we allow more flexibility in the tails of $\epsilon_{t+1}$ by assuming a distribution that allows for fat-tails. We do this by assuming a Student-t density with $\nu_{\epsilon}$ degrees of freedom. For this reason we will refer to it as $\log -t$. Just like the specification in (3.9) does not allow to recognize a density for the return process, in this case the density of $\sigma_{t+1}^{2}$ is not known in closed form. The last specification we consider follows from (3.9) and it is introduced in Corsi, Mittnik, Pigorsch, and Pigorsch (2008). This specification allows for a time varying $\omega$ to be driven by a $\operatorname{GARCH}(1,1)$ dynamic. From now on we will refer to it with the name $V o V$. Its dynamic is given by:

$$
\begin{aligned}
\log \sigma_{t+1}^{2} & =m_{t+1}+\omega_{t+1} \epsilon_{t+1} \\
\omega_{t+1}^{2} & =\kappa_{0}+\kappa_{1} \omega_{t}^{2}+\kappa_{2}\left(h_{t}-m_{t}\right)^{2}
\end{aligned}
$$




\section{TAILORING THE ERRORS}

In this case, the distribution of $\sigma_{t+1}^{2}$ is conditionally log-normal with the moments in equation (3.10) being characterized by a time varying, but non-stochastic, $\omega$.

We evaluate which is a approximately good density of the unexpected component of $\sigma_{t+1}^{2}$ for our model specifications by looking at a set of diagnostic checks that we introduce in the following subsection.

\subsubsection{Diagnostic Checks}

Diebold, Gunther, and Tay (1998) introduce the probability integral transform (PIT) to assess the quality of a given model in density forecasting. For volatility, the PIT is defined as

$$
z_{t+1}=\int_{0}^{\sigma_{t+1}^{2}} g(u) d u
$$

That is the cumulative distribution function of any of the specifications introduced in the previous section that has density $g(\cdot)$. For a correctly specified model $z_{t+1}$ should be iid standard uniform, i.e. $z_{t+1} \sim \mathfrak{U}(0,1)$. There are several ways to assess the sequence of $z_{t+1}$ is iid uniform as pointed out in Hujer and Vuletic (2007). Although informal, one of the most popular way is through a histogram of the PIT sequence. To give an intuition behind this graphical test, suppose to divide the sample containing all values of $z_{t+1}$ in ten different bins. Under the null each bin should contain approximately a tenth of the sample size. On the contrary, if the model that implies $g$ presents some deficiencies in parts of the density than we might observe a butterfly shaped histogram in the case that the model generates too few observations in the tails of the distribution. Confidence bands around the expected number of observations falling into each bin can be computed by recalling that the number of observations falling into each bin is distributed as a Binomial random variable $\mathcal{B}\left(T, \frac{N}{T}\right)$ where $T$ is the number of the observations and $N$ is the number of bins forming the histogram.

Rejection of the null via a Kolmogorov-Smirnov (KS) test can however be due to violation of uniformity, violation of iidness or both. For this reason, we perform a further model assessment by considering the test introduced by Berkowitz (2001). This builds on the PIT by stating that if the model performs reasonably well then

$$
b_{t+1}=\Phi^{-1}\left(z_{t+1}\right) \sim \mathcal{N}(0,1)
$$


where $\Phi^{-1}$ is the standard normal inverse distribution function. As pointed out in the original paper we can test whether the sequence $b_{t+1}$ is iid standard normal. This can be done, for example, by writing down the following autoregressive model:

$$
b_{t+1}-\delta=\rho\left(b_{t}-\delta\right)+\gamma u_{t+1}
$$

The null hypothesis that $b_{t+1}$ is iid standard normal implies that the triplet $\left(\delta, \gamma^{2}, \rho\right)=$ $(0,1,0)$ can be tested via a likelihood ratio test as

$$
L R=-2(\mathcal{L}(0,1,0)-\mathcal{L}(\widehat{\delta}, \widehat{\gamma}, \widehat{\rho}))
$$

which under the null is distributed as a $\chi^{2}(3)$.

\subsubsection{Backtesting methods}

A large number of backtesting-methods exist and for a survey of the existing methods we refer to Nieto and Ruiz (2016) and Berkowitz, Christoffersen, and Pelletier (2011). We focus on two absolute tests, like the one developed by Kupiec for unconditional coverage and the one in Christoffersen and Jacobs (2004) for conditional coverage, and a relative test based on the Model Confidence Set developed by Hansen, Lunde, and Nason (2011). The starting point of all these tests is the so called hit-statistic indicator or violations, $J_{t}$, that takes value equal to one if the return falls below the forecasted VaR and zero otherwise. The test developed from Kupiec assesses the quality of a VaR model based on the number of violations: if it is statistically close to the VaR coverage rate $\alpha$ then the model is deemed acceptable.

The main issue with the above-mentioned test is that it does not take into account a possible dynamic in the time series of the violations. In fact dependence in the violations sequence implies severe capital losses for the institution under consideration. There are many ways to test independence between the violations, we test conditional coverage using the test introduced in Christoffersen and Jacobs (2004). In what follows, we briefly explain the intuition and implementation of the test. For a more detailed discussion we refer to the original paper.

Christoffersen and Jacobs (2004) extend previous work from Christoffersen (1998) by introducing a test to assess more elaborate forms of clustering in the violations. Instead of considering the sequence of violations, this test assesses conditional coverage by looking at the durations, which are defined according to

$$
D_{j}=t_{j}-t_{j-1}
$$




\section{TAILORING THE ERRORS}

with $t_{j}$ being the day with the $\mathrm{j}$-th violation. Christoffersen and Jacobs (2004) show that under the null hypothesis that violations do not cluster, the duration sequence $D$ is expected to be exponentially distributed with parameter $\alpha$. The alternative hypothesis of the violations exhibiting clustering is captured by the duration sequence being Weibull distributed with parameters $\xi$ and $\psi$ and density given by:

$$
f_{W}(D, \xi, \psi)=\psi^{\xi} D^{\xi-1} e^{-(\psi D)^{\xi}}
$$

The associated likelihood is given by

$l(D ; \xi, \psi)=C_{1} \ln S\left(D_{1}\right)+\left(1-C_{1}\right) \ln f\left(D_{1}\right)+\sum_{j=2}^{J} \ln f\left(D_{j}\right)+C_{J} \ln S\left(D_{J}\right)+\left(1-C_{J}\right) \ln f\left(D_{J}\right)$

where $C_{1}$ is a binary variable taking value one when the $\mathrm{j}$-th duration is censored. This happens only for the first and for the last one: the contribution to the likelihood is given by the survival function $S$. From this, testing for conditional coverage and for independence can be done via a likelihood ratio test.

As briefly introduced above, we also test the different specifications relative to each other using the Model Confidence Set (MCS) procedure introduced in Hansen, Lunde, and Nason (2011). Unlike, the above mentioned testing procedures the MCS evaluate each model against the other and eliminates the worst performing model in an iterative manner until a smaller set containing equally performing models is obtained. To make things more precise, let $\mathcal{M}_{0}$ be the set of all $\mathbb{M}$ competing models. The MCS procedure determines the model(s) that are part of the MCS using a loss function, $\mathcal{L}$, and a significance level, $P^{M C S}$, which are specified by the researcher. Literature usually sets this significance level to $10 \%$ or $25 \%$. For what concerns the loss function, we employ the Asymmetric VaR loss function introduced in Gonzalez-Rivera, Lee, and Mishra (2004) that has the form:

$$
\mathcal{L}_{m, t}=\left(r_{t}-\operatorname{Va} R_{m, t}^{\alpha}\right) \times\left(\alpha-\mathbf{1}_{\left(r_{t}-V a R_{m, t}^{\alpha}\right)}\right)
$$

where $V a R_{m, t}^{\alpha}$ is the forecasted VaR for model $m$ for day $t$ and confidence level $\alpha$. The intuition behind the functional form of the loss function in (3.20) is that returns that exceed VaR are weighted more heavily. The iteration procedure goes through the following steps until the MCS is determined. Starting from $k=1$ to $\mathbb{M}, \mathrm{i}$ ) based on the loss function in (3.20) the relative performance, $\pi_{m}$, of model $m$ relative to the others is computed and models are ranked according to it. Then in step ii), using an equivalence test $\mathcal{T}_{\mathcal{M}_{k}}$ the hypothesis $H_{0, \mathcal{M}_{k}}: \pi_{m}=0$ is tested for every model in $\mathcal{M}_{k}$. This hypothesis testing step gives as a by-product a p-value, $P_{H_{0}, \mathcal{M}_{k}}$. The usual interpretation for a p-value applies: a small value for $P_{H_{0}, \mathcal{M}_{k}}$ implies that the null hypothesis that all models in $\mathcal{M}_{k}$ perform 
equally is likely to be rejected. This procedure then reaches step iii): as long as $H_{0}, \mathcal{M}_{k}$ is rejected an elimination rule $\mathcal{E}_{\mathcal{M}_{k}}$ is used to eliminate the model that is statistically more different from the other competing models. The MCS p-value for the model determined by the elimination rule $\mathcal{E}_{\mathcal{M}_{k}}$ is then computed using $P_{\mathcal{E}_{\mathcal{M}_{k}}}=\max _{i \leq k} P_{H_{0}, \mathcal{M}_{k}}$. This makes it easy to conclude whether a model belongs to the MCS or not by comparing $P_{\mathcal{E}_{\mathcal{M}_{k}}}$ to $P^{M C S}$. The MCS p-value then has the interpretation that a model with a $P_{\mathcal{E}_{\mathcal{M}_{k}}}$ lower than $P^{M C S}$ is not part of the set of best performing models. After a model is eliminated the procedure starts again and the three steps are performed again until $H_{0, \mathcal{M}_{k}}$ cannot be rejected anymore.

\section{$3.3 \quad$ Results}

\subsubsection{Data}

Our dataset consists of intraday prices of the SPY, an ETF that tracks the SP500, and intraday prices of 28 current constituents of the Dow Jones starting from November 1995 until November 2015. This leaves us with time series of realized variance of 5036 observations computed using 5-minutes returns. The choice for this sampling frequency follows Liu, Patton, and Sheppard (2015), who confirm that 5-minutes RV obtains a good balance between the bias arising from higher frequenciers and precision that is lost using lower frequencies. However, when it comes to computing daily or multiperiod VaR the overnight return must be considered. This accounts for a significant fraction of the daily realized variance as pointed out by Hansen and Lunde (2005b). They propose an estimator that uses the information embedded in the overnight return and takes a linear combinator of the overnight variance and the Realized Variance obtained from high frequency data:

$$
R V_{t}^{H L}=w_{1} v_{t}^{2}+w_{2} R V_{t}
$$

where $v_{t}^{2}$ is the estimator of the overnight variance obtained as the square overnight return, $w_{1}$ and $w_{2}$ are respectively the weight associated to the two components. ${ }^{3}$

\footnotetext{
${ }^{3}$ These two components can be obtained in a straightforward manner because they require the computation of sample averages and covariances. For some companies this implies that $w_{1}$ becomes negative due to huge outliers happening overnight. In this case we set $w_{1}$ equal to zero, which, as Hansen and Lunde (2005b) point out, is similar to the scaling estimator:
}

$$
R V_{t}^{s c a l}=\kappa R V_{t}
$$


For the dynamic models introduced in the previous section, we then use this estimator as a measure of the daily Integrated Variance:

$$
\sigma_{t}^{2}=R V_{t}^{H L}
$$

Table 4.1 shows the main summary statistics for the returns and of the stocks belonging to our dataset and the last row shows the same statistics for the SPY.

Table 3.1: Return Distribution

\begin{tabular}{lcccccccccc}
\hline & \multicolumn{4}{c}{$\mathrm{r}_{i, t}$} & & \multicolumn{4}{c}{$\mathrm{r}_{i, t} / \sigma_{i, t}$} \\
\cline { 2 - 7 } \cline { 8 - 10 } Stock & Mean & St.dev. & Skew. & Kurt. & & Mean & St.dev. & Skew. & Kurt. \\
Min & 0.009 & 1.229 & -3.357 & 6.783 & & 0.022 & 0.948 & -0.314 & 2.922 \\
0.10 & 0.016 & 1.465 & -1.385 & 7.367 & & 0.032 & 0.980 & 0.007 & 3.007 \\
0.50 & 0.031 & 1.837 & -0.102 & 10.766 & & 0.044 & 1.004 & 0.081 & 3.325 \\
0.90 & 0.053 & 2.504 & 0.139 & 30.839 & & 0.060 & 1.056 & 0.166 & 4.540 \\
Max & 0.086 & 3.034 & 0.198 & 87.024 & & 0.114 & 1.095 & 0.227 & 9.926 \\
Mean & 0.032 & 1.908 & -0.408 & 17.309 & & 0.048 & 1.009 & 0.065 & 3.720 \\
St.dev. & 0.015 & 0.414 & 0.826 & 18.642 & & 0.019 & 0.033 & 0.096 & 1.390 \\
\hline SPY & 0.028 & 1.577 & -0.208 & 12.481 & & 0.059 & 1.003 & 0.005 & 3.002 \\
\hline
\end{tabular}

This table shows the main statistics on the current DJIA constituents. The first four moments are shown for the daily returns and the daily returns standardized by the current volatility. The row that is called Min shows the minimum values of the relevant summary statistics the dataset we have whereas Max shows the maximum values. The second and third last rows show the mean and the standard deviation of the summary statistics in the different columns. We also show the same summary statistics for $\mathrm{r}_{i, t} / œ_{i, t}$.

For the majority of the companies we observe a kurtosis slightly above 3. More specifically, 4 out of 29 present a kurtosis higher than 4 with a maximum reaching 9.92. Andersen, Bollerslev, Diebold, and Labys (2001) and Andersen, Bollerslev, Diebold, and Labys (2003) focus their empirical application on exchange rates and hence do not deal with the overnight.

Andersen, Bollerslev, Frederiksen, and Nielsen (2010) studies some features of the mixture distribution hypothesis considering new non-parametric transformation of the open-to-close return and treat the overnight returns as deterministically occurring jumps. The second set of summary statistics is related to the realized variances, table 4.2 shows the same summary statistics for realized variances and log realized variances.

Clearly, $\log R V$ seems much closer to be normally distributed with the highest kurtosis being 3.71. Few companies in our dataset present a kurtosis below 3 .

Table 3.3 summarizes the estimates for the different specifications for the SPY. From these 
Table 3.2: Volatility Distribution

\begin{tabular}{lcccccccccc}
\hline & \multicolumn{4}{c}{$\sigma_{i, t}^{2}$} & & \multicolumn{4}{c}{$\ln \sigma_{i, t}^{2}$} \\
\cline { 2 - 3 } Stock & Mean & St.dev. & Skew. & Kurt. & & Mean & St.dev. & Skew. & Kurt. \\
Min & 1.370 & 2.919 & 6.120 & 74.030 & & -0.223 & 0.834 & -0.061 & 2.309 \\
0.10 & 2.146 & 3.567 & 7.305 & 97.734 & & 0.148 & 0.879 & 0.269 & 2.657 \\
0.50 & 3.374 & 6.687 & 19.032 & 563.274 & & 0.657 & 0.986 & 0.403 & 3.211 \\
0.90 & 6.270 & 18.021 & 43.783 & 2566.841 & & 1.234 & 1.127 & 0.533 & 3.624 \\
Max & 9.204 & 23.977 & 68.267 & 4777.375 & & 1.555 & 1.183 & 0.593 & 3.723 \\
Mean & 3.874 & 9.101 & 21.596 & 930.846 & & 0.683 & 0.985 & 0.385 & 3.174 \\
St.dev. & 1.725 & 5.823 & 15.904 & 1174.613 & & 0.411 & 0.089 & 0.139 & 0.362 \\
\hline SPY & 1.510 & 2.918 & 9.809 & 164.941 & & -0.247 & 1.093 & 0.265 & 3.019 \\
\hline
\end{tabular}

This table shows the main statistics on the current DJIA Realized Variances constituents. The first four moments are shown for the Realized Variances and the log transformation. The row that is called Min shows the minimum values of the relevant summary statistics the dataset we have whereas Max shows the maximum values. The second and third last rows show the mean and the standard deviation of the summary statistics in the different columns.

elements we can see that the strong persistence, measured by the sum of the autoregressive coefficients, documented in Corsi (2009) and literature thereafter is also present in our data. The multiplicative specification exhibits an estimate of $\nu$ around 7 . This implies that the return distribution for this model is a student-t with the same number of degrees of freedom and scale weighted by a factor around 0.7. Compared to the $L N$ model, the $\log -t$ specification has an additional parameter, $\nu_{\epsilon}$, which controls how heavy tailed volatility shocks are. In our case, we obtain a parameter estimate higher than 17 , therefore resulting in a fatter tail compared to the normal distribution.

For the model with leverage the distinguishing parameter is $\ell$ and as we can see in the third column of the table it has a negative value equal to -0.275 . To better appreciate this effect we present a scatter plot of the standardized return and the volatility shocks in Figure 3.1.

The last column shows the estimates for the specification that has time varying $\omega$ driven by a $\operatorname{GARCH}(1,1)$ process. Comparing our estimates with those in Corsi, Mittnik, Pigorsch, and Pigorsch (2008) we observe that the GARCH parameter has a higher value and at the same time the $\mathrm{ARCH}$ one is lower. ${ }^{4}$ Figure 3.2 shows the filtered time varying $\mathrm{VoV}$ for this model. It is straightforward to notice that this measure is often stable around the unconditional mean and sometimes it exhibits spikes.

\footnotetext{
${ }^{4}$ Corsi, Mittnik, Pigorsch, and Pigorsch (2008) perform their empirical analysis using SP500 futures whereas we use an ETF that tracks the same index
} 
Table 3.3: $S P Y$ models' estimates

\begin{tabular}{lccccc}
\hline Par. & $i G$ & $L N$ & $L e v$ & $L o g-t$ & VoV \\
\hline$\alpha_{0}$ & 0.115 & -0.020 & & -0.020 & -0.020 \\
& $(0.040)$ & $(0.008)$ & & $(0.008)$ & $(0.008)$ \\
$\alpha_{1}$ & 0.274 & 0.437 & & 0.437 & 0.437 \\
& $(0.086)$ & $(0.019)$ & $(0.019)$ & $(0.019)$ \\
$\alpha_{2}$ & 0.387 & 0.339 & 0.339 & 0.339 \\
& $(0.128)$ & $(0.028)$ & & $(0.028)$ & $(0.028)$ \\
$\alpha_{3}$ & 0.240 & 0.182 & & 0.182 & 0.182 \\
& $(0.060)$ & $(0.022)$ & & $(0.022)$ & $(0.022)$ \\
$\omega$ & & 0.512 & & 0.512 & \\
& & $(0.005)$ & & $(0.005)$ & \\
$\nu$ & 6.626 & & & & \\
& $(0.087)$ & & -0.275 & & \\
$\ell$ & & & $(0.087)$ & & \\
& & & & 17.549 & \\
$\nu_{\epsilon}$ & & & & & 0.008 \\
& & & & & $(0.329)$ \\
$\kappa_{0}$ & & & & $0.001)$ \\
& & & & & $(0.004)$ \\
$\kappa_{1}$ & & & & & \\
$\kappa_{2}$ & & & & & \\
& & & & & \\
\hline
\end{tabular}

This table shows the parameters estimates for all the specifications we have considered for the $S P Y$. Standard errors are in brackets and are calculated from the diagonal elements of the matrix $-\left[\frac{\partial^{2} l(\widehat{\theta})}{\partial \theta \partial \theta^{\prime}}\right]^{-1}$, where $l(\widehat{\theta})$ denotes the log-likelihood function evaluated at the ML estimate of $\theta$, the vector of model parameters. 
Figure 3.1: Leverage Effect $S P Y$

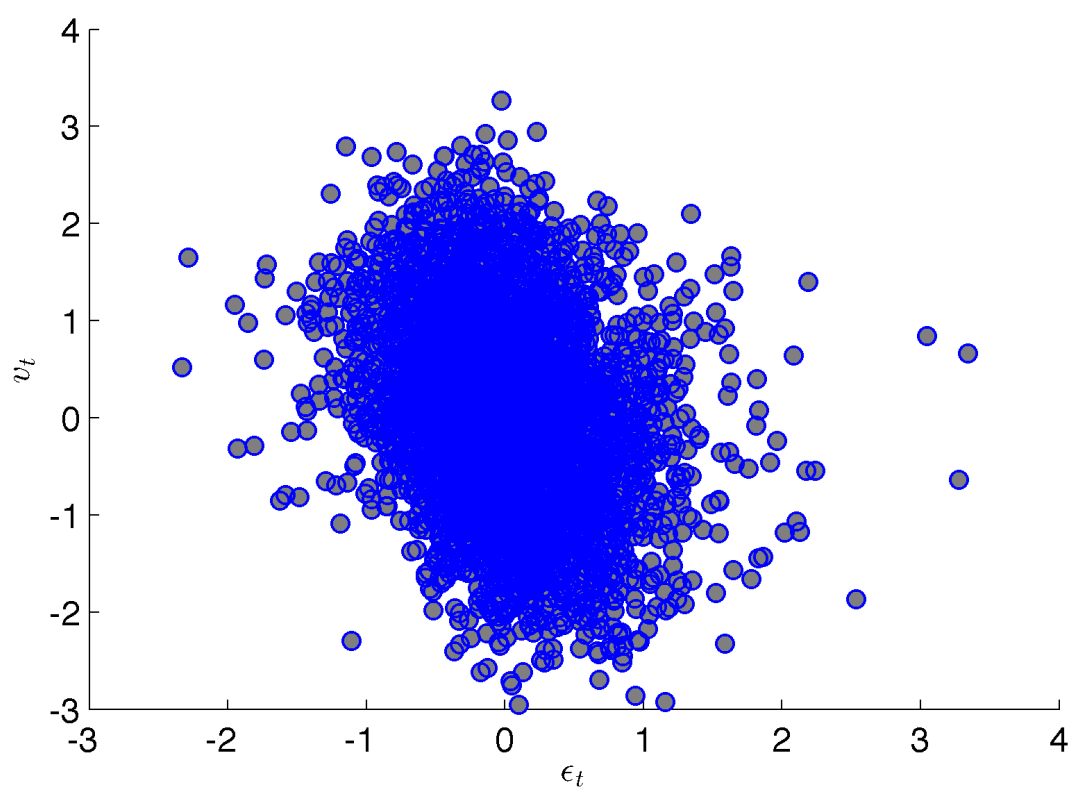

This graph shows a scatter plot of the returns standardized by realized volatility, $v_{t}$, and the shocks to volatility, $\epsilon_{t}$.

Figure 3.2: $\omega_{t}$ estimated from the GARCH specification

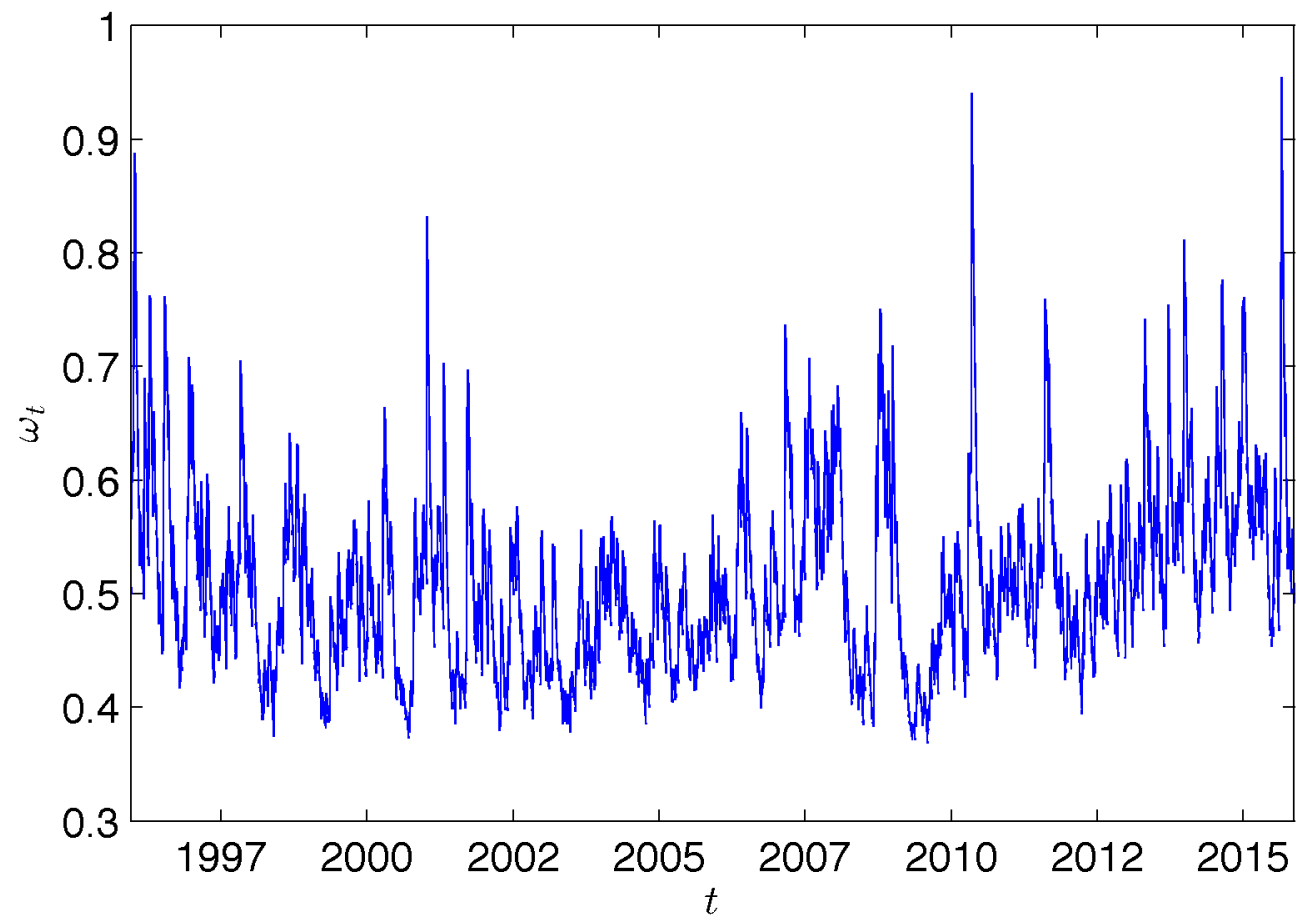

This graph shows the filtered time varying VoV from model $V o V$ over the full sample for the $S P Y$. 


\section{TAILORING THE ERRORS}

Table 3.4 display the estimates of all the models focusing on the characterizing parameters of each specification. Focusing on the first column, we notice that the lowest value for the degrees of freedom parameter is 4.7 and the maximum is slightly below 10 . The companies exhibiting these values are Intel and Coca Cola.

Table 3.4: All companies models' estimates

\begin{tabular}{cccccc}
\hline & $i G$ & $L N$ & Lev & Log $-t$ & $V o V$ \\
& $\nu$ & $\omega$ & $\ell$ & $\nu_{\epsilon}$ & $\frac{\kappa_{0}}{1-\kappa_{1}-\kappa_{2}}$ \\
\hline Min & 4.732 & 0.451 & -0.129 & 13.178 & 0.451 \\
0.25 & 6.828 & 0.468 & -0.078 & 16.526 & 0.469 \\
Med & 7.895 & 0.480 & -0.059 & 18.629 & 0.481 \\
0.75 & 8.526 & 0.498 & -0.047 & 20.485 & 0.498 \\
Max & 9.273 & 0.547 & -0.005 & 22.466 & 0.558 \\
\hline
\end{tabular}

This table shows the parameters estimates for all the specifications we have considered for the all the companies in our dataset. The rows denote respectively the minimum of a parameter estimate, the lower quartile, the median, the upper quartile and finally the maximum.

For the second specification in column two we conclude that there is not much heterogeneity. Related to the estimation of the leverage parameter for the DJIA companies, the third column shows that $\ell$ is negative for all the stocks in our dataset. For this parameter, the lowest value is given by Exxon with a value of -0.129 whereas Nike exhibits the highest value equal to -0.005 . The fourth column presents the estimates for the degrees of freedom for the shocks to volatility. These values range from a minimum of 13 , exhibited by Merck, to a maximum of almost 22, for Exxon. The last three columns present the estimates for the the specification with time varying volatility of volatility. Focusing on the constant for this specification the minimum is estimated for Apple and the maximum for United Health. For the GARCH parameter, $\kappa_{1}$, Caterpillar exhibits the lowest value whereas Merck has the highest. Finally, Merck also exhibits the lowest $\kappa_{2}$ and the highest is estimated for Intel. Also, it is worth mentioning that all the companies result in a stationary model, condition that is checked by the value of $\kappa_{1}+\kappa_{2}$ for all the stocks in the dataset. This sum ranges from a minimum of 0.189 for Caterpillar to a maximum of 0.986 for Apple.

\subsubsection{Diagnostic Checks Results}

In the VaR backtesting part we will split the sample in two parts and use the first part that we denote $\mathrm{T}_{I S}$ to estimate the volatility models. Then we use a second part of the sample, $\mathrm{T}_{O O S}$, to assess the quality of the different models in forecasting daily VaR. In 
this section we consider instead the full sample to draw a conclusion about the different specifications over the entire time span we have at hand.

The following plot presents the result for the PIT applied to the innovations of the four model specifications we have introduced in section one. Each panel of figure 3.3 refers to a different specification: for example, panel (a) reports the PIT for the multiplicative specification with $i G(\nu)$ innovations. The second one refers to the log-normal model whereas panel (c) reports the results for the log specification with $\mathrm{t}\left(\nu_{\epsilon}\right)$ distributed shocks. Finally, panel (d) reports the results of the log-normal model with GARCH innovations. Analyzing each plot itself and starting from the top left, we notice a highly marked butterfly shaped histogram: this means that this specification implies too many observations falling into the center of the innovations' distribution and too few are falling into the the tails. Moving to the second specification we observe that the number of observations is much closer to the required ones. However, all these specifications present a number of observations falling into the left tail which is too low compared to the expected ones. The $V o V$ model is the only one that seems to capture this aspect better than the other ones suggesting that the time varying vol-of-vol has a relevant effect for the left tail of the innovation distribution.

Though instructive this graphical test does not tell us whether the model is misspecified because of the violation to uniformity or due to some misspecified dependence in the PIT sequence. For this reason in the following table we report the value of the KS statistic as well the one of the Likelihood ratio test introduced in Berkowitz (2001).

Table 3.5: Models' diagnostic checks for the $S P Y$

\begin{tabular}{lccccc}
\hline Model & KS & LR & $\delta$ & $\gamma$ & $\rho$ \\
\hline$i G$ & 0.048 & 275.572 & -0.031 & 0.884 & 0.271 \\
$L N$ & 0.031 & 1.645 & 0 & 0.995 & -0.018 \\
Log $-t$ & 0.034 & 43.856 & -0.037 & 0.876 & -0.019 \\
$V o V$ & 0.027 & 6.899 & 0 & 1.001 & -0.037 \\
\hline
\end{tabular}

This table shows some selected diagnostic checks for the SPY for the different specifications. The first column refers to the value of the Kolmogorov-Smirnov test statistic, the second one to the Likelihood Ratio test in (3.16). Last three columns report the estimates of the parameters to be tested.

From table 3.5 we easily observe that the multiplicative specification is rejected by the data. In fact the associated statistic is larger than its critical value being 0.021 . The same can be concluded from the Berkowitz likelihood ratio statistic. This statistic subsumes the joint hypothesis problem that the sequence has zero mean, unity variance and no autocorrelation among the observations. The critical value at $1 \%$ confidence level is 11.35 . 
Figure 3.3: Probability Integral Transform Results

(a) $i G$

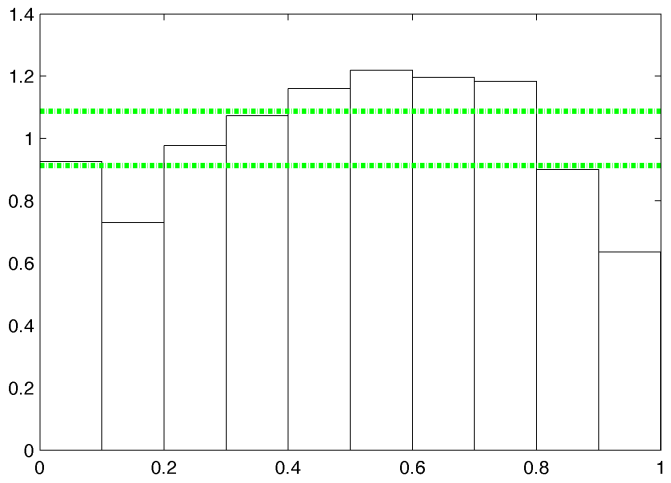

(c) $\log -t$

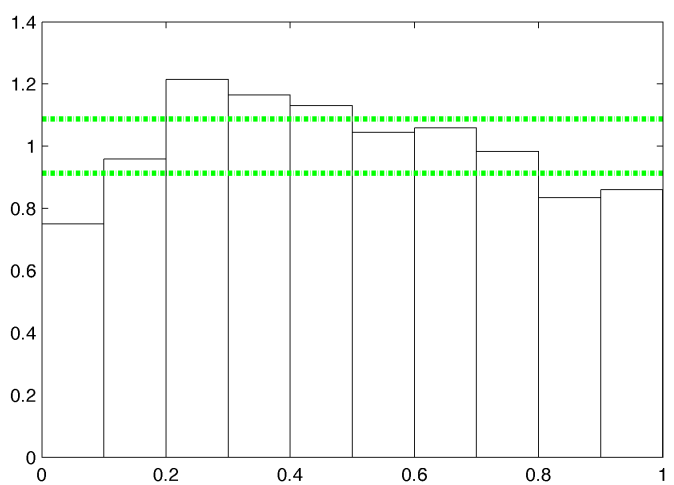

(b) $L N$

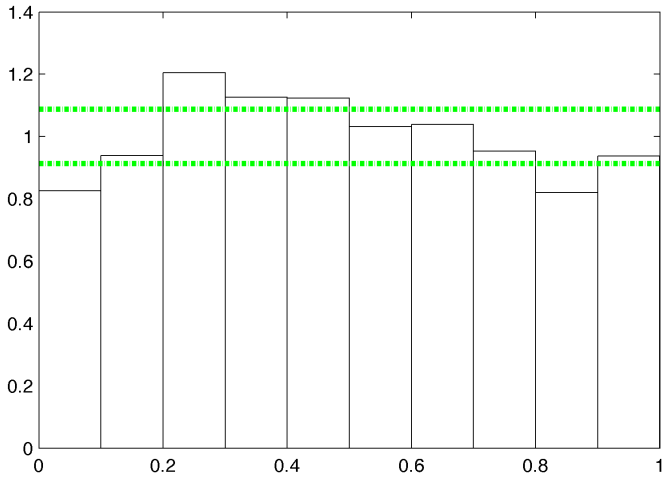

(d) $\mathrm{VoV}$

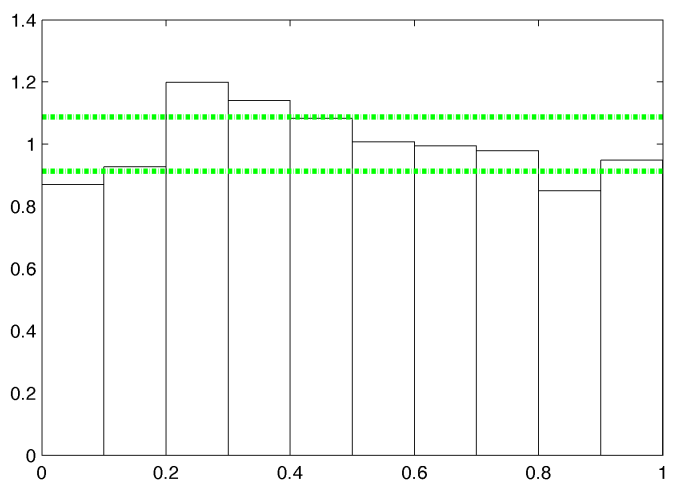

This graph shows (standardized) probability integral transform applied to the innovation of each of the four specifications introduced in section one estimated on the $S P Y$ for the full sample. In green are the confidence bands. Under the null the number of observations falling into each Bin is distributed as a Binomial, $\mathcal{B}\left(T, \frac{N}{T}\right)$ where $N$ is the number of bins and $T$ the total number of observations. 
From the values of these parameters we notice that this specification exhibits both higher mean and autocorrelation when compared to the other ones and a variance much lower than unity. Moving to the specification in logs, we notice that the values of $\delta$ are zero (by construction) for the $L N$ and $V o V$ specifications but the $t\left(\nu_{\epsilon}\right)$ shocks specification exhibits the lowest value. For what concerns the variance's values, we can notice that the model with time varying Vol-of-Vol is closer to unity. Finally, for the autocorrelation parameter, $\rho$, we observe that, again, the lowest is presented by the fourth specification. Next table summarizes the results for the single stocks in our sample focusing only on the values of the parameters in model (3.15). From it, we can quickly assess the quality of each time series model over the cross-section of companies at our disposal. For example, we can easily infer that the inverse gamma is not able to capture the salient features of the data. For instance, the average of the $b_{t+1}$ sequence is far from being zero as reported in the first three columns. This is further corroborated by the fact that also the autocorrelation parameter, $\rho$, shows a cross-sectional dispersion far from being close to the null hypothesis with a median value of 0.151 . For what concerns the variance instead the median appears much closer to the value under the null with a maximum reached at 0.986 .

Table 3.6: Models' diagnostic checks for all the companies in our dataset

\begin{tabular}{|c|c|c|c|c|c|c|c|c|c|}
\hline \multirow[t]{2}{*}{ Model } & \multicolumn{3}{|c|}{$\delta$} & \multicolumn{3}{|c|}{$\gamma$} & \multicolumn{3}{|c|}{$\rho$} \\
\hline & Min & Med & Max & Min & Med & Max & Min & Med & Max \\
\hline$i G$ & -0.080 & -0.047 & -0.014 & 0.537 & 0.918 & 0.986 & 0.034 & 0.151 & 0.635 \\
\hline$L N$ & 0 & 0 & 0 & 0.999 & 1.000 & 1.000 & -0.021 & -0.015 & -0.010 \\
\hline $\log -t$ & -0.007 & -0.003 & -0.002 & 0.822 & 0.880 & 0.904 & -0.025 & -0.017 & -0.009 \\
\hline$V o V$ & -0.006 & -0.001 & 0.002 & 0.997 & 1.000 & 1.001 & -0.028 & -0.019 & -0.009 \\
\hline
\end{tabular}

This table shows the parameter estimates of the Berkowitz model in equation (3.15). For each parameter and each specification, we report the minimum, the median and the maximum. The statistics' values are independently sorted.

Moving to the $L N$ specification from the same table and focusing directly on the last column we can observe that the estimates are all negative with a maximum of $(-0.01)$. The highest value for the likelihood ratio test is 2.27 that compared to the critical value under the null let us conclude that we do not reject this distributional specification for innovations to volatility. For what concerns the $\log -t$ model instead, we can notice that we have a form of misspecification coming from the a mean effect that can be seen from the values of the statistics associated to $\delta$ but more importantly the misspecification mostly affects the variance of the $b_{t+1}$ sequence as it is shown in the columns associated to $\gamma$. Finally, for the model that takes into account the time varying nature of the Vol- 


\section{TAILORING THE ERRORS}

of-Vol we observe similar results to the ones for the $L N$ specification. Also in this case the highest value for the likelihood ratio test is below the critical value associated to the testing problem under the null.

\subsubsection{VaR implications}

Given the results reported in the previous sections, here we present the implications of the different time series models for VaR, which is implicitly defined by:

$$
\alpha=\int_{-\infty}^{-V a R(\alpha)} p\left(r_{t+1} \mid I_{t}\right) d r_{t+1}
$$

In this section we also focus on the $S P Y$ to illustrate how the time series specifications differ from each other. Figure 3.4 shows VaR for the different time series specifications with the confidence level ranging from $1 \%$ to $5 \%$.

Figure 3.4: VaR for different specifications

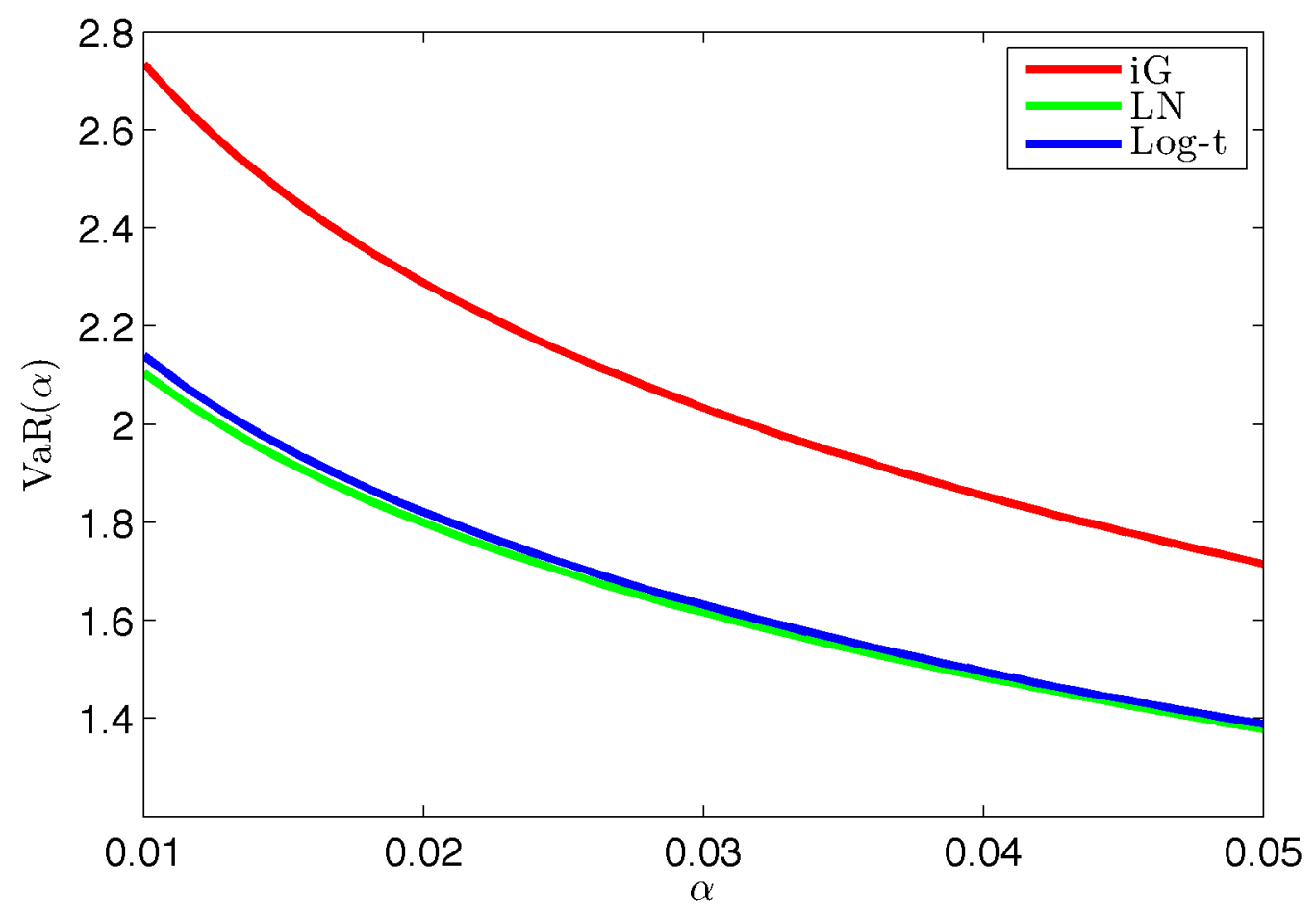

This graph shows the VaR estimated at different confidence levels and for three time series models. $i G$ is the inverted Gamma model, $L N$ is the log-normal model and $L o g-t$ is the model with Student-t errors. The VaR estimates are related to the parameters' values for the $S P Y$.

The specifications $L N$ and $L o g-t$ are hard to distinguish as it is expected given the estimate of $\nu_{\epsilon}$ reported in the previous section. On the other hand, the multiplicative 
specification, $i G$, implies a marked difference with respect to the other models.

The next comparison that we make in this section considers the model with time varying $\mathrm{VoV}$ and with it we compute VaR assuming that $\omega_{t}$ can have three values: low, medium and high. More specifically, we set these values equal to the $10 \%$ quantile of the unconditional distribution of $\mathrm{VoV}$, equal to the median and to the $90 \%$ quantile respectively.

Figure 3.5: VaR for the time varying $\omega$ specification

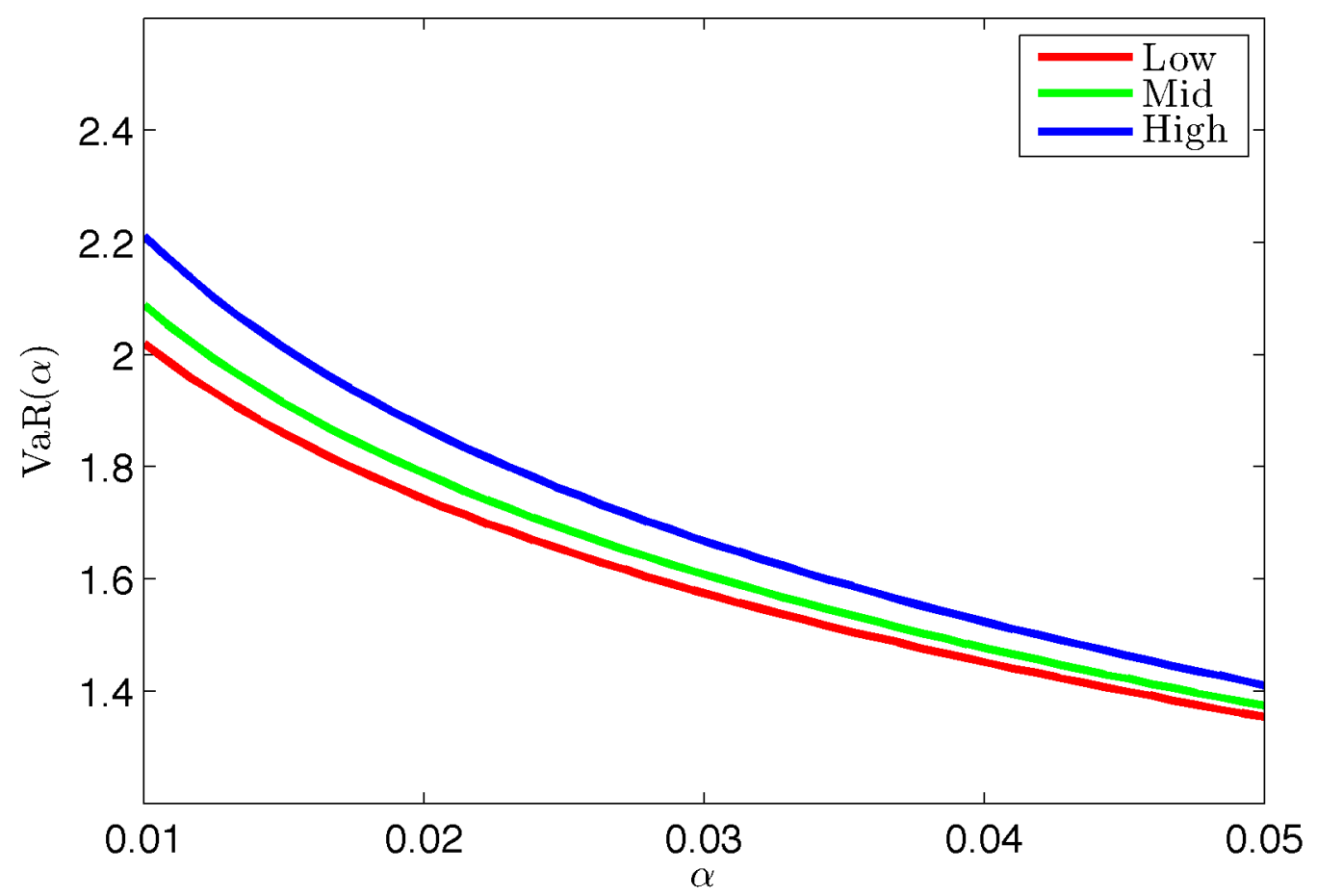

This graph shows the VaR estimated at different confidence levels and for the specification exhibiting time varying VoV. The VaR estimates are related to the parameters' values for the $S P Y$.

To facilitate comparison across the different values of $\omega_{t}$, we assume that $\mu_{t}$ is fixed and equal to the unconditional mean. This is not the case when looking at the time series of $\omega_{t}$ and $\sigma_{t}$, we can notice that high periods of volatility are characterized by high periods of $\mathrm{VoV}$. The effects of time varying $\omega$ on VaR can be seen in figure 3.5. From this, we highlight that, although weak, a more marked difference between the three cases is seen when $\alpha=1 \%$.

The last comparison adds leverage as explained in the methology section. The effects of leverage can be seen in figure 3.6.

From this figure we can notice that the leverage effect has a strong impact on VaR. In fact, this asymmetry pulls the left tail of the return distribution to have more probability mass and subtracts a substantial part of it from the right tail. 
Figure 3.6: VaR for long and short positions
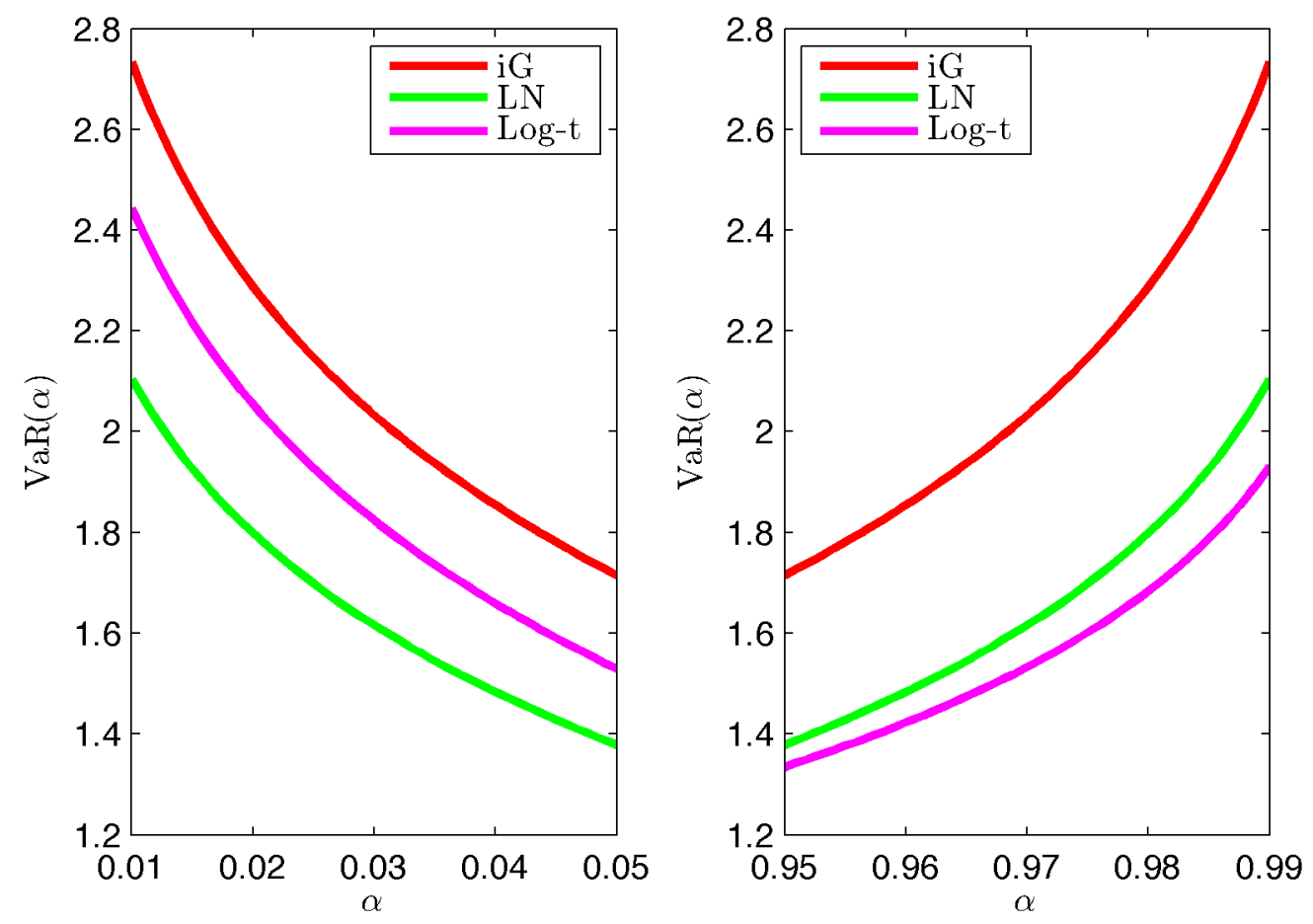

This graph shows the VaR estimated at different confidence levels. The left plot refers to the VaR for long positions whereas the right plot to the VaR for short ones. The VaR estimates are related to the parameters' values for the $S P Y$. 


\subsubsection{Backtesting Results}

Given the results in the previous sections, we now turn to study the performance of the different specifications in forecasting VaR. The quality of the various time series models is then assessed through the backtesting procedures explained in the previous section. We have a total of 1000 observations for the out-of-sample assessment, starting from November 2011. We then have 4000 observations to estimate the various models and issue a VaR forecast for the next day. Then, according the vast literature on forecasting we move the estimation sample of one observation and estimate the models again until the observations in the out-of-sample part are exhausted.

We start by considering the Unconditional Coverage test at two different confidence levels. Table 3.7 reports the results for this testing problem. This table shows the number of violations that each time series model implies, the estimated coverage rate, $\widehat{\alpha}$ and the $p$-values obtained from the likelihood ratio test where under the null the coverage rate is $\alpha$.

Table 3.7: Unconditional Coverage test $S P Y$ for Long positions

\begin{tabular}{lcccccccc}
\hline & \multicolumn{3}{c}{$\alpha=5 \%$} & & \multicolumn{3}{c}{$\alpha=1 \%$} \\
\cline { 2 - 4 } \cline { 6 - 8 } Model & N.Viol & $\widehat{\alpha}$ & $p-v a l$ & & N.Viol & $\widehat{\alpha}$ & $p-v a l$ \\
\hline$i G$ & 47 & 0.046 & 0.590 & & 5 & 0.005 & 0.072 \\
LN & 49 & 0.048 & 0.805 & & 10 & 0.009 & 0.964 \\
Lev & 36 & 0.035 & 0.026 & & 2 & 0.002 & 0.001 \\
Log $-t$ & 48 & 0.047 & 0.695 & & 9 & 0.009 & 0.714 \\
VoV & 42 & 0.041 & 0.197 & & 8 & 0.008 & 0.714 \\
\hline
\end{tabular}

This table shows the results from the Unconditional Coverage test for two confidence levels for the $S P Y$. The columns represent the number of violations, the fraction of the violations and the the p-value associated to the likelihood ratio test.

Focusing first, on $\alpha=5 \%$ under a correct risk model the number of violations should be approximately 50. Regarding the different specifications we notice that for this confidence level all the time series models deliver a satisfactory VaR estimate as highlighted by the high $p$-values. The exception is given by the specification with leverage that results in a too conservative VaR compared to the other models. Moving to $\alpha=1 \%$, we notice that the difference among models becomes more marked. For instance, the multiplicative model and the specifications with leverage and time varying $\mathrm{VoV}$ are overly conservative. On the other hand, the simple log-normal specification exhibit a coverage rate that is fairly close to the required one for $\alpha=1 \%$.

As a further diagnostic of the overall quality of the risk models under consideration we focus on the Conditional Coverage test explained in the previous section. Results from 


\section{TAILORING THE ERRORS}

this test are shown in table 3.8. Focusing on $\alpha=5 \%$, we notice that the average distance between the violations is rather similar for the five risk models, the conditional coverage test implies that the data do not reject any of the models.

Table 3.8: Conditional Coverage test $S P Y$ : Long position

\begin{tabular}{lccccccc}
\hline & \multicolumn{4}{c}{$\alpha=5 \%$} & & \multicolumn{3}{c}{$\alpha=1 \%$} \\
\cline { 2 - 4 } \cline { 6 - 8 } Model & $D_{\min }$ & $\bar{D}$ & $p-$ val & & $D_{\min }$ & $\bar{D}$ & $p-$ val \\
\hline$i G$ & 1 & 21.104 & 0.823 & & 47 & 168.833 & 0.150 \\
LN & 1 & 20.206 & 0.988 & & 3 & 92.091 & 0.888 \\
Lev & 2 & 27.378 & 0.856 & & 159 & 337.666 & 0.998 \\
Log $-t$ & 1 & 20.673 & 0.949 & & 3 & 101.300 & 0.641 \\
VoV & 1 & 23.558 & 0.977 & & 159 & 337.667 & 0.998 \\
\hline
\end{tabular}

This table shows the results from the Conditional Coverage test for two confidence levels for the $S P Y$. The columns represent the minimum value of the duration between two violations, $D_{\min }$, the average duration, $\bar{D}$, and the $p$-value associated to the likelihood ratio test.

At $\alpha=1 \%$ the difference among models becomes more relevant even though the data still do not reject any of the five specifications.

As a final diagnostic check we check which models are in the Model Confidence Set. This testing procedure is based on a block bootstrap algorithm and we set the number of bootstrap replications equal to 10000. Unlike the other two tests, this procedure compares the size of the losses for each pair of models and eliminates from the set the model(s) that perform poorly relative to the others. Table 3.9 reports the results for the models contained in the MCS.

Table 3.9: Model Confidence Set for the $S P Y$ : Long position

\begin{tabular}{lrrrrrrr}
\hline & \multicolumn{3}{c}{$\alpha=5 \%$} & & \multicolumn{3}{c}{$\alpha=1 \%$} \\
\cline { 2 - 3 } \cline { 6 - 8 } E.R. & $P_{H_{0, \mathcal{M}_{k}}}$ & $P_{\mathcal{E}_{\mathcal{M}_{k}}}$ & E.M. & & $P_{H_{0, \mathfrak{M}_{k}}}$ & $P_{\mathcal{E}_{\mathcal{M}_{k}}}$ & E.M. \\
\hline$e_{\mathcal{M}_{1}}$ & 0.000 & 0.000 & $i G$ & & 0.000 & 0.000 & $i G$ \\
$e_{\mathcal{M}_{2}}$ & 0.189 & 0.189 & $L e v$ & & 0.010 & 0.010 & $L e v$ \\
$e_{\mathcal{M}_{3}}$ & 0.397 & 0.397 & $L o g-t$ & & 0.005 & 0.010 & $L o g-t$ \\
$e_{\mathcal{M}_{4}}$ & 0.382 & 0.397 & $L N$ & & 0.782 & 0.782 & $L N$ \\
$e_{\mathcal{M}_{5}}$ & 1.000 & 1.000 & $V o V$ & & 1.000 & 1.000 & $V o V$ \\
\hline
\end{tabular}

This table shows the MCS procedure results for the $S P Y$ at two distinct confidence levels. The columns represent the elimination rule E.R, the $k-t h$ set p-value, $P_{H_{0, \mathfrak{M}_{k}}}$, the MCS p-value, $P_{\mathcal{E}_{\mathcal{M}_{k}}}$ and the eliminated model at iteration $k$, E.M.

Focusing on the testing problem when $\alpha=5 \%$ we can infer that the multiplicative specification is not part of the MCS as it is the first model to be eliminated. For a $p^{M C S}$ of 
$10 \%$, all the specifications in logs are part of the MCS. When $\alpha=1 \%$, the multiplicative specification is the first one to be eliminated, followed by the model with leverage and the one with time varying $\mathrm{VoV}$. In this case, the log-normal model and the log-t one are part of the MCS.

\subsubsection{Short Positions}

As explained in the previous sections, the introduction of leverage effect allows to have a different VaR for the left and right tail of the returns distribution. In this section, we focus on the results for the right tail. This is of interest for investors that short-sell a given security and therefore are also interested in a tail loss, which in this case arises from an extreme positive return.

We start by showing the results for the Unconditional Coverage test in table 3.10. For both confidence levels, we can see that the specification with leverage is the closest to the nominal coverage rate. For the $1-\alpha=5 \%$ case, it is worth highlighting that the simple log specifications provide satisfactory results. On the other hand, when we approach the right tail of the distribution the specification with leverage results in a satisfactory number of violations, whereas the other ones are overly conservative.

Table 3.10: Unconditional Coverage test $S P Y$ : Short position

\begin{tabular}{lcccccccc}
\hline & \multicolumn{3}{c}{$1-\alpha=5 \%$} & & \multicolumn{3}{c}{$1-\alpha=1 \%$} \\
\cline { 2 - 5 } \cline { 6 - 8 } Model & N.Viol & $1-\widehat{\alpha}$ & $p-v a l$ & & N.Viol & $1-\widehat{\alpha}$ & $p-v a l$ \\
\hline$i G$ & 31 & 0.031 & 0.002 & & 1 & 0.001 & 0.000 \\
LN & 44 & 0.043 & 0.324 & & 2 & 0.002 & 0.001 \\
Lev & 49 & 0.048 & 0.805 & & 6 & 0.005 & 0.157 \\
Log $-t$ & 41 & 0.040 & 0.149 & & 2 & 0.002 & 0.001 \\
VoV & 36 & 0.036 & 0.026 & & 1 & 0.001 & 0.000 \\
\hline
\end{tabular}

This table shows the results from the Unconditional Coverage test for two confidence levels for the SPY for Short positions. The columns represent the number of violations, the fraction of the violations and the the p-value associated to the likelihood ratio test.

To corroborate this findings, we again use the MCS procedure and the results can be found in table 3.11.

Table 3.11 confirms the results from the unconditional coverage test. The specification with leverage is the last model to be eliminated from the Model Confidence Set implying that the size of the losses for this specification are the smallest when compared to the other time series models. From the left part of the table we can see that the the logspecifications, except for the one with time varying $\mathrm{VoV}$, are all part of MCS. Moving to 
Table 3.11: Model Confidence Set for the $S P Y$ : Short position

\begin{tabular}{lrrrrrrrr}
\hline & \multicolumn{3}{c}{$1-\alpha=5 \%$} & & \multicolumn{3}{c}{$1-\alpha=1 \%$} \\
\cline { 2 - 3 } \cline { 7 - 8 } E.R. & $P_{H_{0, \mathcal{M}_{k}}}$ & $P_{\mathcal{E}_{\mathcal{M}_{k}}}$ & E.M. & & $P_{H_{0, \mathcal{M}_{k}}}$ & $P_{\mathcal{E}_{\mathcal{M}_{k}}}$ & E.M. \\
\hline$e_{\mathcal{M}_{1}}$ & 0.000 & 0.000 & $i G$ & & 0.000 & 0.000 & $i G$ \\
$e_{\mathcal{M}_{2}}$ & 0.004 & 0.004 & $V o V$ & & 0.001 & 0.001 & $V o V$ \\
$e_{\mathcal{M}_{3}}$ & 0.536 & 0.536 & $L o g-t$ & & 0.029 & 0.029 & $L o g-t$ \\
$e_{\mathcal{M}_{4}}$ & 0.624 & 0.624 & $L N$ & & 0.064 & 0.064 & $L N$ \\
$e_{\mathcal{M}_{5}}$ & 1.000 & 1.000 & $L e v$ & & 1.000 & 1.000 & Lev \\
\hline
\end{tabular}

This table shows the MCS procedure results for the $S P Y$ at two distinct confidence levels. The columns represent the elimination rule E.R, the $k-t h$ set p-value, $P_{H_{0, \mathcal{M}_{k}}}$, the MCS p-value, $P_{\mathcal{E}_{\mathcal{M}_{k}}}$ and the eliminated model at iteration $k$, E.M.

the case for $1-\alpha=1 \%$, the only specification being part of the MCS is the one with leverage confirming the results from table 3.10 .

\subsubsection{Individual Stocks}

This section reports the results for the companies in our dataset. In order to summarize the results across all companies we report the rejection rates for the unconditional and conditional coverage statistic, and the average MCS p-value for each model. More specifically, for each model the rejection rate is computed as follows:

$$
R R_{s}=\frac{1}{N} \sum_{i=1}^{N} \mathbf{1}_{\text {pval }_{i}^{s}<p}
$$

where $s$ is either UC or CC, which respectively stand for Unconditional Coverage or Conditional Coverage. Clearly, (3.22) is bounded between zero and one. If $R R$ is close to one means that a specific model is often rejected by the data in the cross-section of companies. On the other hand, if this is low the reverse hold. The second measure that we report is the cross-sectional average of the MCS p-values. This measure is bounded between zero and one. A high value for this statistic implies that a given model is most likely to be in the MCS of all the companies in the cross-section.

Tables 3.12 and 3.13 report the statistics introduced above for long and short positions respectively. Focusing on the former we observe that the for what concerns the Unconditional Coverage test, the multiplicative specification is the most rejected one in the cross section at both confidence levels. The results for the Conditional Coverage corroborate the findings obtained for the $S P Y$. None of the models is rejected on the basis of this test. Focusing on the last two columns, we observe that the models that are most likely to be in the MCS in the cross-section is logarithmic specification with time varying VoV and 
the simple log-normal model. This finding holds at both confidence levels $\alpha$.

Table 3.12: Robustness Statistics All Stocks: Long position

\begin{tabular}{|c|c|c|c|c|c|c|}
\hline \multirow[t]{2}{*}{ Model } & \multicolumn{2}{|c|}{$R R_{U C}$} & \multicolumn{2}{|c|}{$R R_{C C}$} & \multicolumn{2}{|c|}{$\widetilde{P}_{\mathcal{M}}$} \\
\hline & $\alpha=5 \%$ & $\alpha=1 \%$ & $\alpha=5 \%$ & $\alpha=1 \%$ & $\alpha=5 \%$ & $\alpha=1 \%$ \\
\hline$i G$ & 0.393 & 0.214 & 0.000 & 0.000 & 0.003 & 0.044 \\
\hline$L N$ & 0.071 & 0.036 & 0.000 & 0.000 & 0.330 & 0.546 \\
\hline Lev & 0.142 & 0.071 & 0.000 & 0.000 & 0.145 & 0.036 \\
\hline $\log -t$ & 0.071 & 0.071 & 0.000 & 0.000 & 0.158 & 0.047 \\
\hline $\mathrm{VoV}$ & 0.036 & 0.000 & 0.000 & 0.000 & 0.975 & 0.796 \\
\hline
\end{tabular}

This table shows the summary statistics for the backtests for the companies in our cross-section for a long position. The columns represent the different statistics and different confidence levels $\alpha$.

Turning to table 3.13, we again highlight the poor performance of the multiplicative specification. In fact, this is the most rejected specification when it comes to the Unconditional Coverage test. For this backtesting procedure the other specifications deliver

Table 3.13: Robustness Statistics All Stocks: Short position

\begin{tabular}{|c|c|c|c|c|c|c|}
\hline \multirow[t]{2}{*}{ Model } & \multicolumn{2}{|c|}{$R R_{U C}$} & \multicolumn{2}{|c|}{$R R_{C C}$} & \multicolumn{2}{|c|}{$\widetilde{P}_{\mathcal{M}}$} \\
\hline & $\alpha^{\star}=5 \%$ & $\alpha^{\star}=1 \%$ & $\alpha^{\star}=5 \%$ & $\alpha^{\star}=1 \%$ & $\alpha^{\star}=5 \%$ & $\alpha^{\star}=1 \%$ \\
\hline$i G$ & 0.571 & 0.143 & 0.000 & 0.000 & 0.104 & 0.077 \\
\hline$L N$ & 0.036 & 0.036 & 0.000 & 0.000 & 0.181 & 0.105 \\
\hline Lev & 0.000 & 0.036 & 0.000 & 0.000 & 0.768 & 0.790 \\
\hline $\log -t$ & 0.036 & 0.071 & 0.000 & 0.000 & 0.141 & 0.084 \\
\hline$V o V$ & 0.036 & 0.071 & 0.000 & 0.000 & 0.583 & 0.464 \\
\hline
\end{tabular}

This table shows the summary statistics for the backtests for the companies in our cross-section for a short position. The columns represent the different statistics and different confidence levels $\alpha^{\star}=1-\alpha$. For short positions $\alpha=95 \%$ and $99 \%$.

approximately the same results at $\alpha^{\star}=5 \%$, in the other case the least rejected models are the logarithmic specification and the one with leverage. For what concerns the Conditional Coverage test, the results are in line with the ones obtained for the long position. Turning to the columns related to the MCS, we observe that the specification with the highest averaged MCS p-values is the model with leverage for both confidence levels $\alpha^{\star}$. 


\subsection{Conclusions}

Volatility play a predominant role in financial decision making, be it for portfolio allocation or risk management. Focusing on the latter aspect, we investigate what is an appropriate mixing distribution for Realized Volatility in terms of daily VaR forecasts. We find that satisfactory VaR forecasts are given by the models in logs, be it either a simple log-normal model as in Andersen, Bollerslev, Diebold, and Labys (2001) or its extension with time varying Vol-of-Vol as in Corsi, Mittnik, Pigorsch, and Pigorsch (2008) for long positions. On the other hand, when considering the right tail of the returns distribution we observe that the best performing model is the log-normal one with leverage effect.

Although banks are interested in relatively short term VaR, financial institutions like pension funds and insurance companies may not given that they have a much longer investment horizon. The present framework could then be used to tackle the problem of temporal aggregation of $\mathrm{VaR}$ for very long horizons. In this case the strong volatility persistence will be fundamental in the determination of the multi-period VaR.

Although we have analyzed the problem of univariate risk measures estimation, risk management issues are of multivariate nature. The determination of VaR for a portfolio of assets therefore requires a thorough understanding of how VaR of the single positions aggregate. With this framework, we could then compute the co-crash probabilities between asset classes and hence assess the strength of diversification benefits in a portfolio. 


\section{Appendix}

\section{Leverage Effect}

Let the mixture model be defined by :

$$
\begin{aligned}
& r=\phi+\sigma v \\
& h=m+\omega \epsilon
\end{aligned}
$$

For the moment we assume $\phi=0$. We assume that volatility is lognormal. Specifically, let $h=\ln \sigma^{2}$, and assume that $h \sim \mathcal{N}\left(m, \omega^{2}\right)$. Moreover, let the leverage effect be specified by a correlation $\ell$ between $v$ and $\epsilon$. We are interested in the marginal distribution of $r$. The transformation from $v$ to $r$ has a Jacobian

$$
J=\frac{\partial v}{\partial r}=\frac{1}{\sigma}=e^{-h / 2}
$$

Therefore the joint density of $(r, h)$ is

$$
p(r, h) \propto e^{-h / 2} \exp \left(-\frac{1}{2} x^{\prime} \Omega^{-1} x\right)
$$

with

$$
\begin{aligned}
& x^{\prime}=\left(\begin{array}{ll}
e^{-h / 2} r & h-m
\end{array}\right) \\
& \Omega=\left(\begin{array}{cc}
1 & \ell \omega \\
\ell \omega & \omega^{2}
\end{array}\right)
\end{aligned}
$$

Using the inverse

$$
\Omega^{-1}=\frac{1}{\omega^{2}\left(1-\ell^{2}\right)}\left(\begin{array}{cc}
\omega^{2} & -\ell \omega \\
-\ell \omega & 1
\end{array}\right)
$$

the quadratic form $Q=x^{\prime} \Omega^{-1} x$ can be expanded as

$$
\begin{aligned}
Q & =\frac{r^{2}}{e^{h}\left(1-\ell^{2}\right)}-2 \frac{\ell(h-m) r}{e^{h / 2} \omega\left(1-\ell^{2}\right)}+\frac{(h-m)^{2}}{\omega^{2}\left(1-\ell^{2}\right)} \\
& =\frac{1}{e^{h}\left(1-\ell^{2}\right)}\left(r-\frac{\ell e^{h / 2}(h-m)}{\omega}\right)^{2}+\frac{(h-m)^{2}}{\omega^{2}}
\end{aligned}
$$


Substituting (A.27) in the density (A.26) gives

$$
\begin{aligned}
p(r, h) & \propto \frac{1}{\sqrt{e^{h}\left(1-\ell^{2}\right)}} \exp \left\{-\frac{1}{2} \frac{\left(r-\frac{\ell e^{h / 2}(h-m)}{\omega}\right)^{2}}{e^{h}\left(1-\ell^{2}\right)}\right\} \times \exp \left(-\frac{1}{2} \frac{(h-m)^{2}}{\omega^{2}}\right) \\
& \propto p(r \mid h) \times p(h)
\end{aligned}
$$

(the factor $\left(1-\ell^{2}\right)^{-1 / 2}$ can be added in the proportionality, as it does not depend on $(r, h))$. This shows that conditional on $h$,

$$
r \sim \mathcal{N}\left(\frac{\ell e^{h / 2}(h-m)}{\omega}, e^{h}\left(1-\ell^{2}\right)\right)
$$

To compute this density numerically we can draw $h$ from its marginal (normal) distribution and evaluate the conditional normal density of $r$ at gridpoints $R_{i}$.

The correlation between the shocks to returns and log volatility implies a conditional mean correction in the return distribution. With negative $\ell$ we will tend to find larger negative $r$ when $h$ is big. That produces negative skewness in the marginal distribution of $r$. Negative $\ell$ also leads to a negative unconditional mean for $r$. This unintended mean effect was noted by $\mathrm{Yu}$ (2005), who used this as an argument against this leverage specification. The mean effect is, however, easily corrected by adding a non-zero $\phi$ in (A.23). If we set

$$
\phi=-\mathbb{E}\left[\frac{\ell e^{h / 2}(h-m)}{\omega}\right]
$$

then the unconditional mean for $r$ is zero. In general we can choose $\phi$ to obtain any desired value for the mean return. 


\section{Additional Figures}

Figure 3.A.1: Probability Integral Transform Results

(a) $i G$

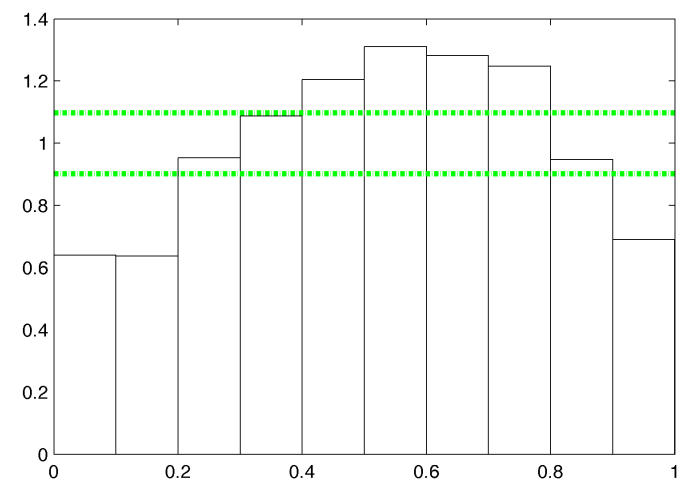

(c) $\log -t$

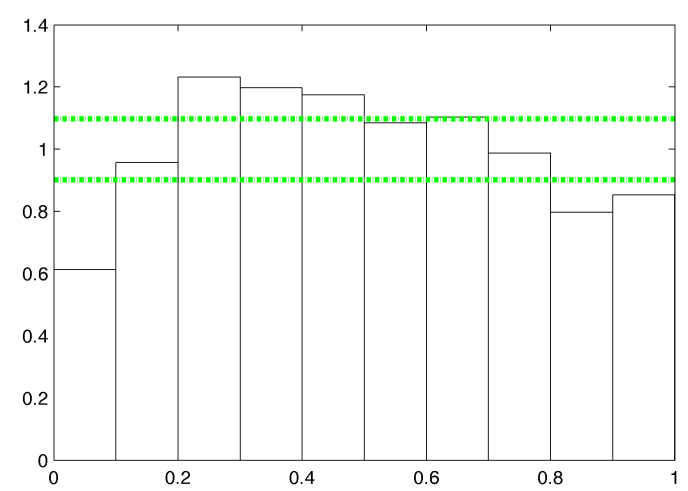

(b) $L N$

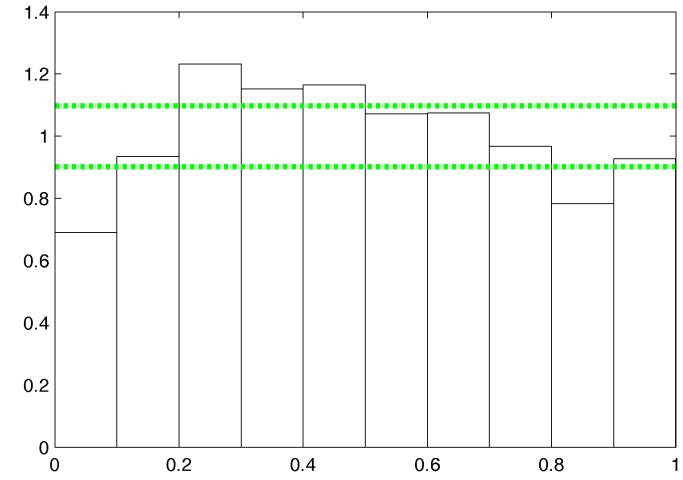

(d) $\mathrm{VoV}$

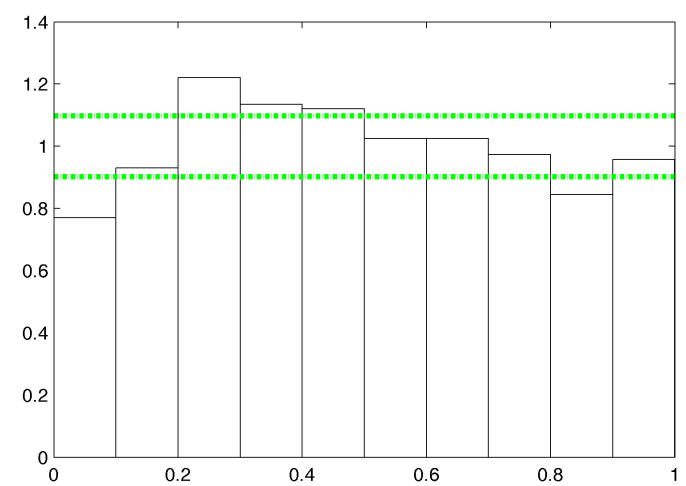

This graph shows (standardized) probability integral transform applied to the innovation of each of the four specifications introduced in section one estimated on the $S P Y$ over a smaller part of the sample including $T_{I S}$ observations. In green are the confidence bands. Under the null the number of observations falling into each Bin is distributed as a Binomial, $\mathcal{B}\left(T, \frac{N}{T}\right)$ where $N$ is the number of bins and $T$ the total number of observations. 


\section{Chapter 4}

\section{Annual VaR From High Frequency Data* $^{*}$}

\subsection{Introduction}

The development of appropriate models and techniques for determining and managing risks is of utmost importance to have a solid financial system. For this reason regulators demand financial institutions to provide measures of risk in case of extreme market turmoil.

These institutions can be distinguished on the basis of their investment horizon: banks, for example, are characterized by a relatively short one whereas pension funds and insurance companies have a much longer one. This difference has important implications to risk management: if on one hand the daily or ten days Value-at-Risk (VaR) is the standard in the banking industry, for the second category it is not useful as regulators demand an annual estimate to prevent an excessive risk-taking behavior.

The literature has been very active in developing models for the daily VaR as shown in Kuester, Mittnik, and Paolella (2006) and Nieto and Ruiz (2016), yet the implications for long horizons such as a year have been almost disregarded. Our aim is to investigate the issue of multi-period VaR aggregation when the investment horizon is one year. However, computing the annual VaR comes with the drawback that the number of non-overlapping observations shrinks dramatically hence making impossible to study the tail of the returns distribution in a non-parametric manner.

Our approach to temporal aggregation builds on the results in Andersen, Bollerslev, Diebold, and Ebens (2001), who find that stock returns are approximately normal when standardized by Realized Volatility computed using intraday data. Although this assumption is empirically valid, the raw returns exhibit heavy tails, which we obtain via

\footnotetext{
*This chapter is based on joint work with Peter Schotman.
} 
the mixture model introduced in Andersen, Bollerslev, Diebold, and Labys (2003) and also adopted in Beltratti and Morana (2005), where the predictive density of returns is obtained as a mixture of the conditional normal density and the distribution of Integrated Variance. This modeling strategy bears an important advantage over existing approaches based on daily data: conditional normality of the returns distribution holds at any aggregation horizon hence annual VaR is characterized by the mixing density, the distribution of Integrated Variance. To obtain this density, we adopt two different time series specifications to capture the persistence in volatility: the first one is a pure Fractional process and the second one is the Heterogeneous AutoRegressive (HAR) model introduced in Corsi (2009).

Other approaches to multi-period VaR are investigated in Embrechts, Kaufmann, and Patie (2005). Their focus is also on the computation of annual VaR but their methodologies are all based on daily data employed either at a daily frequency or at a lower frequency. Among these models, the GARCH framework is considered. Temporal aggregation in a GARCH model is more cumbersome as the distribution of multi-period returns is not known as shown in Drost and Nijman (1993) unless very restrictive assumptions are made on the GARCH structure.

Our approach is also motivated by the findings in Maheu and McCurdy (2011) who point out that using High-Frequency data improves on density forecasting compared to approaches based on daily data solely.

Two fundamental properties of volatility appear important in the determination of annual VaR. Our results stress the importance of estimating annual VaR in a conditional manner: due to the time variation in volatility, the tail of the predicted annual return distribution changes given the most recent market information. This is at the odds of a pure Extreme Value Theory approach where the multi-period VaR is constant therefore neglecting the current market situation.

Our results stress the importance of volatility persistence for $\mathrm{VaR}$ aggregation. In fact, this property allows to shed light on the doubts that Christoffersen and Diebold (2000) cast on the usefulness of volatility for long term risk management purposes. They argue the strength of volatility forecasting diminishes with the increasing investment horizon. We agree with this statement as the prediction error variance increases with the investment horizon. However, within the mixture model the growing uncertainty about variance is taken into account therefore resulting in a more heavy tailed return distribution the longer the investment horizon.

A different approach to multi-period $\mathrm{VaR}$ is given by the usage of a so called scaling law, which links the daily VaR to its annual counterpart. The usage of a scaling law is criticized in Christoffersen, Diebold, and Schuermann (1998), on the basis of mean 
reversion in volatility: it would exacerbate a risk-taking behavior when volatility is low and a too conservative one when volatility is high. Danielsson and Zigrand (2006) criticize this approach showing that it severely underestimates multi-period VaR if the data generating process exhibits jumps. However, Christensen, Oomen, and Podolskij (2014) have highlighted that jumps occurrence has a limited contribution to Realized Variance. Therefore we do not explicitly model jumps in prices. In a GARCH setting, Wang, Yeh, and Cheng (2011) attribute little importance to volatility clustering in relation to failure of the square root of time rule. Our results instead indicate that even for the monthly horizon the square root of time rule underestimates VaR significantly. This is not only due to the strong volatility persistence but also to the increasing uncertainty in volatility. To the light of the above mentioned results, we derive a scaling law, controlling for persistence and the current volatility level, and find that our model delivers a scaling higher than the square root of time rule. Although derived using a different methodology, the results in McNeil and Frey (2000) also point in the direction that a scaling of order higher than square root is needed. We assess the usefulness of the mixture model and our approximate scaling law in comparison with the best performing models in Embrechts, Kaufmann, and Patie (2005). The results confirm that annual VaR should be computed in a conditional manner considering the strong persistence in volatility.

The rest of the chapter is organized as follows: section two introduces the methodology and the econometrics behind it. Section three presents the results and section four concludes the paper. 


\subsection{Methodology}

We assume that the log price, $p_{s}$, of a stock follows the continuous time semi-martingale

$$
d p_{s}=\beta_{s} d s+\nu_{s} d W_{s}
$$

where $W_{s}$ is a standard Brownian motion and $\beta_{s}$ and $\nu_{s}$ denote the drift and the instantaneous volatility processes indipendent of $W_{s}$. Integrating over $s$ it follows that the return over an interval of length $\tau$ is given by

$$
R_{t+\tau}=\int_{t}^{t+\tau} d p_{s}=\int_{t}^{t+\tau} \beta_{s} d s+\int_{t}^{t+\tau} \nu_{s} d W_{s}
$$

When $\tau$ equals 1 day we have the daily return, a quantity most often studied in the literature. In theorem 2 of Andersen, Bollerslev, Diebold, and Ebens (2001) they show that conditional on the volatility process $\nu_{s}, R_{t+\tau}$ is normal with Integrated Variance

$$
S_{t+\tau}^{2}=\int_{t}^{t+\tau} \nu_{s}^{2} d s
$$

However, the Integrated Variance in (4.3) cannot be observed and therefore we need a proxy for it. For $\tau=1$, we write $S_{t+1}^{2}=\sigma_{t+1}^{2}$, and use Realized Variance computed using High Frequency data as an estimator for $\sigma_{t+1}^{2}$. The properties of this quantity are studied extensively in Andersen, Bollerslev, Diebold, and Ebens (2001) for equity returns. They also highlight that the scaled daily returns, $\frac{R_{t+1}}{\sigma_{t+1}}$, appears to be normally distributed. This property is the starting point of our modeling strategy as it allows consistent temporal aggregation: if returns are conditionally normal on a daily frequency then this property also holds at the annual one. This is not the case in a GARCH framework where the distribution of multi-period returns is not known, as pointed out in Drost and Nijman (1993).

Although we assume that the conditional density of returns given current volatility is normal, the unconditional density is known to be leptokurtic. A heavy-tailed distribution of (unscaled) returns is obtained via the mixture model of Andersen, Bollerslev, Diebold, and Labys (2003), where the predictive density of returns is obtained as the integral of the product of the conditional normal density and the density of the Integrated Variance. More formally

$$
p\left(R_{t+\tau} \mid F_{t}\right)=\int f\left(R_{t+\tau} \mid S_{t+\tau}^{2}\right) g\left(S_{t+\tau}^{2} \mid F_{t}\right) d S_{t+\tau}^{2}
$$

where $f(\cdot)$ denotes the normal density, $g(\cdot)$ is the density of Integrated Variance and $F_{t}$ refers to the information set available up to time $t$. With this density we can compute VaR and other risk measures and study how these scale with $\tau$. We focus on VaR, which 
is defined by the property

$$
\alpha=\int_{-\infty}^{-\operatorname{VaR}_{\varnothing}(\alpha)} p\left(R_{t+\tau} \mid F_{t}\right) d R_{t+\tau}
$$

where $\alpha$ is the confidence level chosen to compute VaR. Using a Mixture model we obtain a VaR estimate that is fully characterized by the density of the Integrated Variance, $g\left(S_{t+\tau}^{2}\right)$, obtained employing a dynamic model for Realized Variance. Next section introduces the time series models we adopt and analyzes their properties.

\subsubsection{Time series models}

In what follows we assume a time series model for the logarithm of Realized Variance and we denote it by $h_{t+1}=\ln \sigma_{t+1}^{2}$. This choice is motivated by the empirical findings of Andersen, Bollerslev, Diebold, and Labys (2001) who find that log-normality is a reasonable assumption for the unconditional density of Realized Variance. Realized Variance is known to be a highly persistent process, implying that high variance periods will long last in time therefore having important consequences for the determination of VaR. We capture this empirical feature by means of two dynamic models, which differ in the degree of persistence.

Our first model is the HAR model introduced in Corsi (2009), which mimics the long memory property of Realized Variance in a parsimonious way:

$$
h_{t+1}=\mu+a_{1}\left(h_{t}-\mu\right)+a_{2}\left(h_{t}^{w}-\mu\right)+a_{3}\left(h_{t}^{m}-\mu\right)+\omega \eta_{t+1}
$$

where $\mu$ is the unconditional mean, $\eta_{t+1}$ is standard normally distributed and $\omega$ is the Volatility of Volatility parameter. Furthermore, $h_{t}^{w}$ is the average of the log Realized Variance of the previous five days whereas $h_{t}^{m}$ is the average of the log Realized Variance of the previous twenty one days.

Our second model is nested in the $\operatorname{ARFIMA}(p, d, q)$ class of models. Andersen, Bollerslev, Diebold, and Labys (2003) adopt this model to study the time series properties of Realized Variance. This model can be written as:

$$
a(L)(1-L)^{d} h_{t+1}=\mu+\omega \eta_{t+1}
$$

where $d$ is the fractional parameter and $a(L)$ drives the short term dynamic. Given that our purpose is to study the aggregation for long investment horizons, a pure Fractional 
model is already a reasonable choice. This specification is obtained rewriting (4.7)

$$
h_{t+1}=\mu+\sum_{s=1}^{\infty} b_{s}\left(h_{t+1-s}-\mu\right)+\omega \eta_{t+1}
$$

with the infinite order autoregressive weights $b_{s}$ given by the binomial expansion for the Fractional coefficients. This model is stationary for $d \in(0,0.5)$ and non-stationary but mean reverting for $d \in[0.5,1)$.

With these two dynamic specifications at hand we can now obtain the density of annual return using the mixture model. Time series properties of $h_{t}$ will be important in scaling daily risk measures to longer horizons.

\subsubsection{Long Term VaR Implications}

Our ultimate goal is to determine the tail properties of the predictive density $p\left(R_{t+\tau}\right)$. Following McNeil, Frey, and Embrechts (2015) it is known that this density inherits the tail properties of the mixing distribution, $g\left(S_{t+\tau}^{2}\right)$. In our modeling framework the mixing distribution results from the sum of log-normal random variables and therefore does not have a known density. For this reason, we obtain the predictive density in (4.4) via Monte Carlo simulation. Although $g\left(S_{t+\tau}^{2}\right)$ does not resemble any known density, its properties can be analyzed to the light of the results in Asmussen and Rojas-nandayapa (2008). Their Theorem 1 allows to characterize the tail of the sum of correlated log-normal random variables. This theorem states that the tail behavior of $g\left(S_{t+\tau}^{2}\right)$ is the same as the right tail of the log-normal element with the largest variance. In our case, this is the last element of the series $h_{t+1}, \cdots, h_{t+\tau}$, as the variance of the prediction error increases with $\tau$.

Therefore, if $g\left(S_{t+\tau}^{2}\right)$ can well be approximated by a log-normal then for a NormalLognormal model as in (4.4) follows that multi-period return have conditional excess kurtosis given by

$$
\frac{\mathbb{E}_{t}\left[R_{t+\tau}^{4}\right]}{3 \mathbb{E}_{t}\left[R_{t+\tau}^{2}\right]^{2}}=\exp \left(\mathbb{V}_{t} S_{t+\tau}^{2}\right)
$$

Therefore, the heaviness of the tail is driven by the conditional Vol-of-Vol, $\mathbb{V}_{t}\left[S_{t+\tau}^{2}\right]$, which in the case of a stationary process for $h_{t+1}$ reaches an asymptote for large $\tau$. On the other hand, for a non-stationary process, the Vol-of-Vol diverges implying that the return distribution becomes more fat tailed the longer the investment horizon $\tau$.

From the arguments above, we have highlighted how the annual return distribution is characterized by the time series properties of the volatility processes. Therefore, given 
our setup we can derive an approximate scaling law. A scaling law links the $\tau$ period VaR to its daily one and is derived from the model assumptions. More formally a scaling law has the following form:

$$
\operatorname{VaR}_{\tau}(\alpha)=\tau^{\xi} \operatorname{VaR}_{1}(\alpha)
$$

where $\operatorname{VaR}_{1}(\alpha)$ is the daily $\operatorname{VaR}$ estimate and $\tau^{\xi}$ is the scaling factor needed to obtain the multi-period VaR.

The most common rule is the square root of time rule, which is obtained by setting $\xi=\frac{1}{2}$. This case results from the assumption that the spot volatility process is constant over time. This fact is directly seen by setting $\nu_{s}=\nu$ implying a multi-period Integrated Variance equal to $\tau \nu^{2}$. Using the mixture model this case is prevented to the light of the properties explained in the previous section: using a stochastic volatility model we obtain deviation from normality due to the contribution of the Vol-of-Vol. Furthermore, non-stationary volatility has direct implications on the scaling factor of VaR. The longer the investment horizon $\tau$ implies that the return distribution becomes more heavy tailed. Therefore, the scaling factor would not stabilize with $\tau$ but instead would increase. On the other hand, under a stationary volatility process the required scaling for daily VaR would flatten the longer the investment horizon.

\subsection{Results}

\subsubsection{Data}

Our dataset consists of intraday prices of the SPY, an ETF that tracks the SP500, and intraday prices of 28 constituents of the Dow Jones starting from November 1995 until November 2015. Using intraday return we obtain the Realized Variance as:

$$
R V_{t}=\sum_{i=1}^{n} r_{i, t}^{2}
$$

This leaves us with time series of Realized Variance of 5036 observations computed using 5-minutes returns. The choice for this sampling frequency follows Liu, Patton, and Sheppard (2015), who show that the 5-minutes frequency provides a good balance between the bias arising from higher frequencies and precision lost using lower frequencies. However, when it comes to computing daily or multiperiod VaR the overnight return variance must be considered as it accounts for a significant fraction of the daily Realized Variance as pointed out by Hansen and Lunde (2005b). Unfortunately, for the estimation of the overnight variation high frequency data are not available and therefore we employ the optimal estimator introduced by Hansen and Lunde (2005b). They propose an estimator 
that uses the information embedded in the overnight return and takes a linear combinator of the overnight variance and the Realized Variance obtained from high frequency data:

$$
R V_{t}^{H L}=w_{1} v_{t}^{2}+w_{2} R V_{t}
$$

where $v_{t}^{2}$ is the estimator of the overnight variance obtained as the square overnight return, $w_{1}$ and $w_{2}$ are respectively the weight associated to the two components. ${ }^{2}$

For the dynamic models introduced in the previous section, we then use this estimator as a measure of the daily Integrated Variance:

$$
\sigma_{t}^{2}=R V_{t}^{H L}
$$

Table 4.1 shows the main summary statistics for the returns and of the stocks belonging to our dataset and the last row shows the same statistics for the SPY.

Table 4.1: Returns Distribution

\begin{tabular}{lccccccccc}
\hline & \multicolumn{4}{c}{$\mathrm{r}_{i, t}$} & & \multicolumn{4}{c}{$\mathrm{r}_{i, t} / \sigma_{i, t}$} \\
\cline { 2 - 4 } \cline { 9 - 11 } Stock & Mean & St.dev. & Skew. & Kurt. & & Mean & St.dev. & Skew. & Kurt. \\
Min & 0.009 & 1.229 & -3.357 & 6.783 & & 0.022 & 0.948 & -0.314 & 2.922 \\
0.10 & 0.016 & 1.465 & -1.385 & 7.367 & & 0.032 & 0.980 & 0.007 & 3.007 \\
0.50 & 0.031 & 1.837 & -0.102 & 10.766 & & 0.044 & 1.004 & 0.081 & 3.325 \\
0.90 & 0.053 & 2.504 & 0.139 & 30.839 & & 0.060 & 1.056 & 0.166 & 4.540 \\
Max & 0.086 & 3.034 & 0.198 & 87.024 & & 0.114 & 1.095 & 0.227 & 9.926 \\
Mean & 0.032 & 1.908 & -0.408 & 17.309 & & 0.048 & 1.009 & 0.065 & 3.720 \\
St.dev. & 0.015 & 0.414 & 0.826 & 18.642 & & 0.019 & 0.033 & 0.096 & 1.390 \\
\hline SPY & 0.028 & 1.577 & 0.089 & 12.481 & & 0.059 & 1.003 & 0.093 & 3.002 \\
\hline
\end{tabular}

This table shows the main statistics on the current DJIA constituents. The first four moments are shown for the daily returns and the daily returns standardized by the current volatility. The row that is called Min shows the minimum values of the relevant summary statistics the dataset we have whereas Max shows the maximum values. The second and third last rows show the mean and the standard deviation of the summary statistics in the different columns. We also show the same summary statistics for $\mathrm{r}_{i, t} / \aleph_{i, t}$.

\footnotetext{
${ }^{2}$ These two components can be obtained in a straightforward manner because they require the computation of sample averages and covariances. For some companies this implies that $w_{1}$ becomes negative due to huge outliers happening overnight. In this case we set $w_{1}$ equal to zero, which, as Hansen and Lunde (2005b) point out, is similar to the scaling estimator:
}

$$
R V_{t}^{s c a l}=\kappa R V_{t}
$$


For most companies we observe a kurtosis slightly above 3. More specifically, 4 out of 29 present a kurtosis higher than 4 with a maximum reaching 9.92. For the SPY we notice that conditional normality is a reasonable approximation. All in all, results in Table 4.1 are in line with those presented in Andersen, Bollerslev, Diebold, and Ebens (2001).

Table 4.2: Volatility Distribution

\begin{tabular}{lccccccccc}
\hline & \multicolumn{4}{c}{$\sigma_{i, t}^{2}$} & & \multicolumn{4}{c}{$\ln \sigma_{i, t}^{2}$} \\
\cline { 2 - 3 } Stock & Mean & St.dev. & Skew. & Kurt. & & Mean & St.dev. & Skew. & Kurt. \\
Min & 1.370 & 2.919 & 6.120 & 74.030 & & -0.223 & 0.834 & -0.061 & 2.309 \\
0.10 & 2.146 & 3.567 & 7.305 & 97.734 & & 0.148 & 0.879 & 0.269 & 2.657 \\
0.50 & 3.374 & 6.687 & 19.032 & 563.274 & & 0.657 & 0.986 & 0.403 & 3.211 \\
0.90 & 6.270 & 18.021 & 43.783 & 2566.841 & & 1.234 & 1.127 & 0.533 & 3.624 \\
Max & 9.204 & 23.977 & 68.267 & 4777.375 & & 1.555 & 1.183 & 0.593 & 3.723 \\
Mean & 3.874 & 9.101 & 21.596 & 930.846 & & 0.683 & 0.985 & 0.385 & 3.174 \\
St.dev. & 1.725 & 5.823 & 15.904 & 1174.613 & & 0.411 & 0.089 & 0.139 & 0.362 \\
\hline SPY & 1.510 & 2.918 & 9.809 & 164.941 & & -0.247 & 1.093 & 0.265 & 3.019 \\
\hline
\end{tabular}

This table shows the main statistics on the current DJIA Realized Variances constituents. The first four moments are shown for the Realized Variances and the log transformation. The row that is called Min shows the minimum values of the relevant summary statistics the dataset we have whereas Max shows the maximum values. The second and third last rows show the mean and the standard deviation of the summary statistics in the different columns.

Andersen, Bollerslev, Frederiksen, and Nielsen (2010) studies some features of the mixture distribution hypothesis considering new non-parametric transformation of the open-to-close return and treat the overnight as a deterministically occurring jumps. For our data instead, we consider the daily return as the sum of the intraday one and the overnight one therefore explicitly encompassing the risk deriving from the overnight return.

The second set of summary statistics is related to the Realized Variance, Table 4.2 shows summary statistics for Realized Variance and log Realized Variance. These result corroborate the findings in Andersen, Bollerslev, Diebold, and Labys (2001) regarding lognormality of Realized Variance. In fact, this transformation seems much closer to be normally distributed with the highest kurtosis being 3.72. Regarding the cross section of companies we can see that the cross-sectional average is roughly 3.17 and a minimum of 2.30 .

We present the parameters' estimates for the dynamic models for log Realized Variance. The HAR model can be estimated by OLS. For the Fractional model we use the Local 
Whittle Estimator as suggested in Shimotsu and Phillips $(2006)^{3}$. Table 4.3 shows the parameters' estimates for the $S P Y$ and two individual stocks. The individual stocks differ in their volatility persistence. Focusing on the $S P Y$, the Fractional parameter $d$ lies in the non-stationarity region. ${ }^{4}$ For the HAR model we can compare the parameters for the SPY. Qualitatively our estimates are comparable to the ones obtained by Corsi (2009). The furthest lags get a lower weight compared to the first one.

Table 4.3: Parameter estimates

\begin{tabular}{lccccccc}
\hline Par. & \multicolumn{3}{c}{ HAR } & & \multicolumn{3}{c}{ FI } \\
\cline { 2 - 3 } \cline { 6 - 8 } & Low & High & SPY & & Low & High & SPY \\
$\mathrm{d}$ & & & & & 0.562 & 0.661 & 0.593 \\
$a_{1}$ & 0.334 & 0.301 & 0.437 & & & \\
$a_{2}$ & 0.334 & 0.395 & 0.339 & & & \\
$a_{3}$ & 0.276 & 0.282 & 0.182 & & & \\
$\mu$ & 0.093 & 0.855 & -0.464 & & & \\
$\omega$ & 0.511 & 0.469 & 0.512 & & 0.517 & 0.489 & 0.514 \\
\hline
\end{tabular}

This table shows the estimates of the parameters of the models that we consider for the three constituents of DJIA and for the SPY. The second to fourth columns show the values of the parameters for the HAR model and for the different degrees of persistence. Columns six to nine show the parameter values for the Fractional model. For the estimation of $d$, the number of frequencies used is set equal to $J=T^{\gamma}$ with $\gamma=0.6$. The asymptotic standard error is then given by $s e(d)=1 / \sqrt{4 J}$, which in our case is equal to 0.039 .

The stock characterized by the lowest persistence is Merck and Co., the medium one is Home Depot and the high one is Walmart. Therefore, for all companies at our disposal we find that the fractional parameter lies in the non-stationarity region.

\subsubsection{Distribution of Integrated Variance}

Since $g\left(S_{t+\tau}^{2} \mid I_{t}\right)$ is a conditional density, we must evaluate it given a current state. For this reason we choose the median in the sample. We start by considering the case of the $S P Y$ and then we will return to the companies that present the highest and lowest persistence. Figure 4.1 shows the kernel densities of the simulated Integrated Variance scaled by the investment horizon for both time series specifications for two different $\tau$ : one day and one year. Furthermore, the plot also includes a log-normal fitted to $g\left(S_{t+\tau}^{2}\right)$.

\footnotetext{
${ }^{3}$ We have estimated the fractional model also using the Exact Whittle Estimator and the standard GPH algorithm. The estimates still lie in the non-stationary region

${ }^{4}$ To check the stability of this finding we have set the cutoff parameter $\gamma=0.5,0.7$. With these values, the resulting estimates of $d$ are respectively 0.602 and 0.575 . This implies that the sample average is not a consistent estimator of the unconditional mean variance. We replace $\mu$ with the median, $m$, for both models. Values for $d$ in the non-stationary region in the context of Realized Variances have been found by Rossi and Santucci de Magistris (2014) and Luciani and Veredas (2015).
} 
Figure 4.1: Kernel Densities of Integrated Variance under different models

(a) $\tau=1$

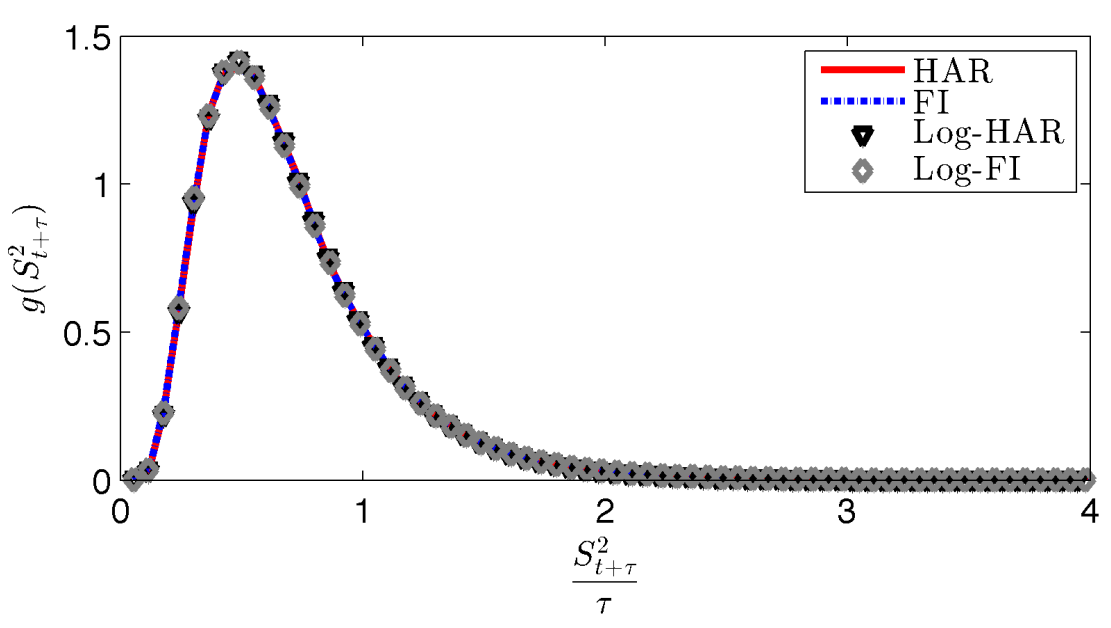

(b) $\tau=252$

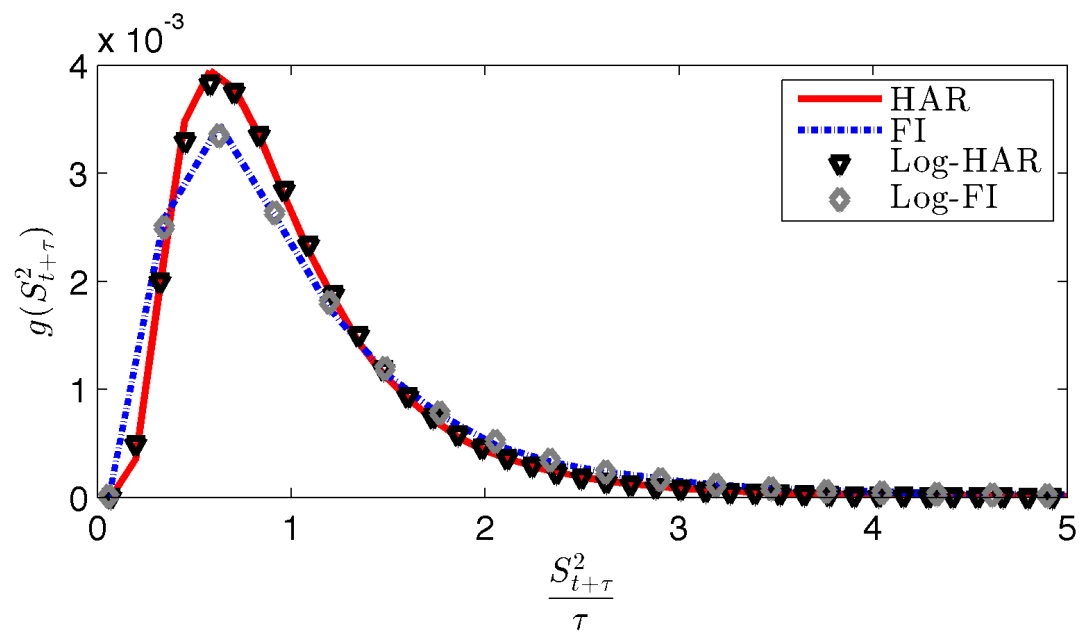

This graph shows the kernel density of the Integrated Variance simulated for the models that we consider for the SPY. The different panels display the change in these density as a function of $\tau$. The triangles are log-normal fits to the densities. 
From this graph we can draw two main conclusions. First, it is clear the evolution of the densities through time: by assumption for $\tau=1$ the Integrated Variance distribution boils down to a $\log$-normal random variable. As $\tau$ increases, $S_{t+\tau}^{2}$ is the sum of log-normal random variables and as such has an unknown density. From Figure 4.1 it appears this sum is well approximated by a log-normal random variable.

From these graphs we can also grasp some features about the two time series specifications we have adopted for $h_{t}$. When $\tau=1$ the models are hard to distinguish, suggesting that for such a short horizon dynamics have a small impact on the predictive density of returns. The difference among the distributions arises from the different values of $\omega$, which affects both the mean and the variance of the distribution of $\sigma_{t+1}^{2}$. As $\tau$ increases, the model with longer memory presents a fatter tailed distribution. This fact is explained by looking at Table 4.4, which shows the conditional mean and variance of the Integrated Variance distribution scaled by the aggregation horizon. The HAR model always exhibits a lower

Table 4.4: Conditional moments of the Integrated Variance Distribution

\begin{tabular}{lccccc}
\hline $\boldsymbol{\tau}$ & \multicolumn{2}{c}{ HAR } & & \multicolumn{2}{c}{ FI } \\
\cline { 2 - 3 } \cline { 5 - 6 } \cline { 5 - 6 } & $\mathbb{E}_{t}\left(S_{t+\tau}^{2} \mid m\right) / \tau$ & $\mathbb{V}_{t}\left(S_{t+\tau}^{2} \mid m\right) / \tau$ & & $\mathbb{E}_{t}\left(S_{t+\tau}^{2} \mid m\right) / \tau$ & $\mathbb{V}_{t}\left(S_{t+\tau}^{2} \mid m\right) / \tau$ \\
\cline { 2 - 3 } 5 & 0.714 & 0.391 & & 0.715 & 0.393 \\
21 & 0.744 & 0.338 & & 0.763 & 0.401 \\
63 & 0.796 & 0.387 & & 0.842 & 0.493 \\
126 & 0.880 & 0.533 & & 1.010 & 0.620 \\
252 & 0.948 & 0.633 & & 1.106 & 0.728 \\
\hline
\end{tabular}

This table shows the scaled moments of the Integrated Variance distributions simulated for the models we consider for the SPY. The different rows display the moments as a function of $\tau$. The first two columns display the moments for the HAR model and the last two for the Fractional model.

scaled mean compared to the Fractional model: this is due to the time series properties of the Fractional model, which is a more persistent process than the HAR model. Also relevant to analyze is the difference in the second moments of the density for the different models: the Fractional model generates a higher volatility of volatility compared to the HAR model.

Figure 4.2 illustrate these effects. Panel A summarizes the results from the previous table for the first moment of the Integrated Variance distribution: the difference between the two models becomes more evident when a higher $\tau$ is considered. Panel B shows the scaled Vol-of-Vol: the Fractional model exhibits a monotonically upward sloped behavior whereas the HAR model starts flattening after the six months horizon. The upward sloping Vol-of-Vol for the fractional model is due to the mildly nonstationary dynamics 
Figure 4.2: Moments of the Integrated Variance distribution scaled by the horizon $\tau$

(a) $\mathbb{E}_{t}\left(S_{t+\tau}^{2}\right)$

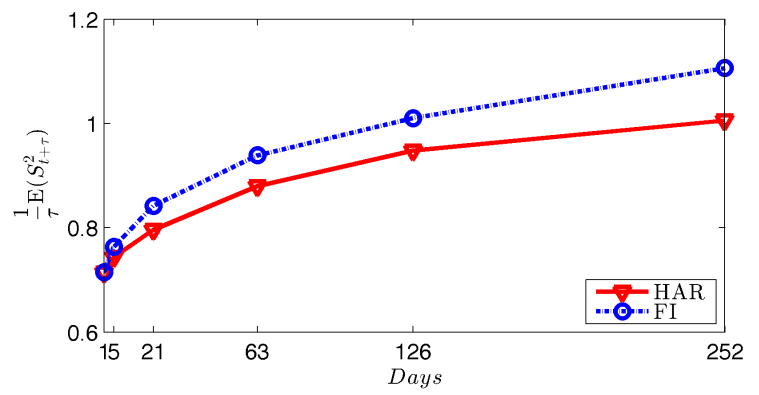

(b) $\mathbb{V}_{t}\left(S_{t+\tau}^{2}\right)$

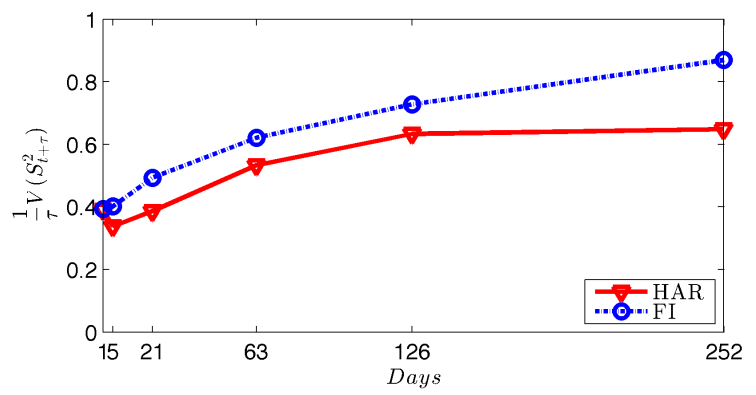

This graph shows the first two moments of the Integrated Variance distributions simulated for the models for the SPY scaled by the horizon $\tau$. Panel A shows the conditional expectation of Integrated Variance under the different models whereas panel B shows the standard deviation both scaled by $\tau$.

arising from $d>\frac{1}{2}$. Therefore, the Vol-of-Vol, $\mathbb{V}_{t}\left[S_{t+\tau}^{2}\right]$ grows with $\tau$.

A key element in the aggregation of VaR for any $\tau$ is then given by the persistence of the time series model that stems from their parametrization. For the Fractional this means a weaker or stronger scaling depending on the value of $d$. In what follows we briefly show the results on the stocks selected according to their persistence as in table 4.3. The following plots show the first two moments of $S_{t+\tau}^{2}$ for Merck and Walmart for the Fractional model. 5 The value of $d$ matters as the company that presents a higher $d$ exhibits a much steeper

Figure 4.3: Moments of the Integrated Variance distribution for different persistence levels

(a) $\mathbb{E}_{t}\left(S_{t+\tau}^{2}\right)$

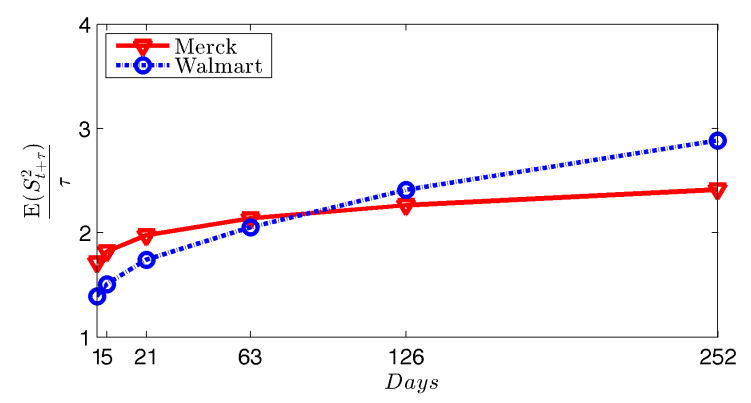

(b) $\mathbb{V}_{t}\left(S_{t+\tau}^{2}\right)$

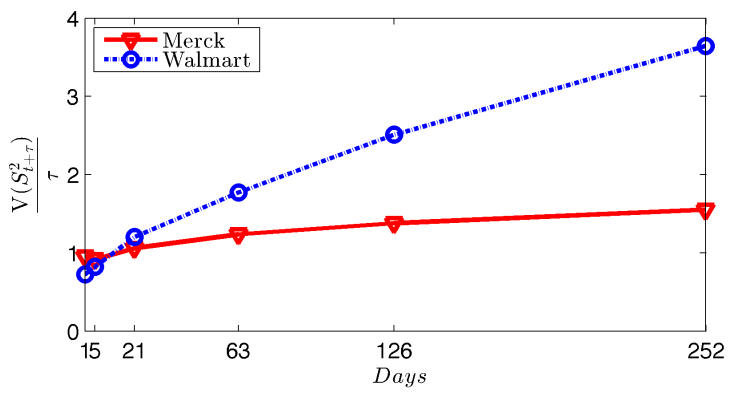

This graph shows the first two moments of the Integrated Variance distributions for Merck and Walmart scaled by the horizon $\tau$.

growth compared to the other one.

\footnotetext{
${ }^{5}$ Qualitatively for the HAR they show the same kind of behavior as shown in table 4.A.1 in the Appendix.
} 


\subsubsection{Long Term VaR}

Building on the previous arguments we compute $\mathrm{VaR}_{\tau}$ for different horizons considering several relevant aspects. Starting from the $S P Y$, we consider the effects on VaR of the current volatility state, being either high or low. Furthermore, we analyze the implications of persistence on VaR aggregation.

\subsubsection{SPY}

We start focusing on $\mathrm{VaR}$ for the $S P Y$. This case is interesting as it represents a portfolio composed by the major US companies.

Table 4.5: VaR for $S P Y$ at different horizons

\begin{tabular}{lcccccccc}
\hline$\tau$ & \multicolumn{3}{c}{ HAR } & & \multicolumn{3}{c}{ FI } \\
\cline { 2 - 4 } \cline { 6 - 8 } & $\alpha=5 \%$ & $\alpha=1 \%$ & $\alpha=0.5 \%$ & & $\alpha=5 \%$ & $\alpha=1 \%$ & $\alpha=0.5 \%$ \\
\cline { 2 - 5 } \cline { 6 - 8 } 1 & 1.377 & 2.102 & 2.403 & & 1.377 & 2.105 & 2.406 \\
5 & 3.154 & 4.715 & 5.347 & & 3.185 & 4.838 & 5.518 \\
21 & 6.680 & 10.048 & 11.430 & & 6.840 & 10.517 & 12.058 \\
63 & 12.090 & 18.669 & 21.448 & & 12.470 & 19.476 & 22.469 \\
126 & 17.721 & 27.674 & 31.973 & & 18.248 & 28.805 & 33.405 \\
252 & 25.796 & 40.160 & 46.264 & & 26.873 & 43.033 & 50.215 \\
\hline
\end{tabular}

This table shows the VaR at two confidence levels computed for the different models for the $S P Y$. The different rows display the aggregation horizon ranging from one day, first row, to one year, last row. All VaR are computed at the median volatility level.

Table 4.5 shows VaR computed for the different models and for several horizons. When $\tau=1$. The table also shows that at any confidence level the Fractional model implies a more conservative VaR than the HAR model. The difference between the two models becomes more evident when longer horizons are considered.

As we have introduced in the methodology section, an alternative approach to obtain multi-period VaR is to scale up the daily one with the square root of time rule. Therefore, it is interesting to compare our $\mathrm{VaR}_{\tau}$ estimates to the ones obtained with the square root of time rule. Figure 4.4 shows long term VaR scaled by $\sqrt{\tau}$. At all confidence levels and for both time series specifications the square root of time rule delivers a less conservative long term VaR. The deviations from this rule are a result of the time series properties of volatility. Both moments grow faster than $\tau$ : the fact that the conditional mean grows faster has the direct implication that the square root of time rule is violated and the growth in the conditional variance makes the return distribution more leptokurtic. This second fact can be seen by studying the differences from the two time series specifications. Up to one year horizon the HAR approximates the Fractional model reasonably well. If we 
Figure 4.4: $\operatorname{VaR}_{\tau}$ and the $\sqrt{\tau}$ rule

(a) $\alpha=5 \%$

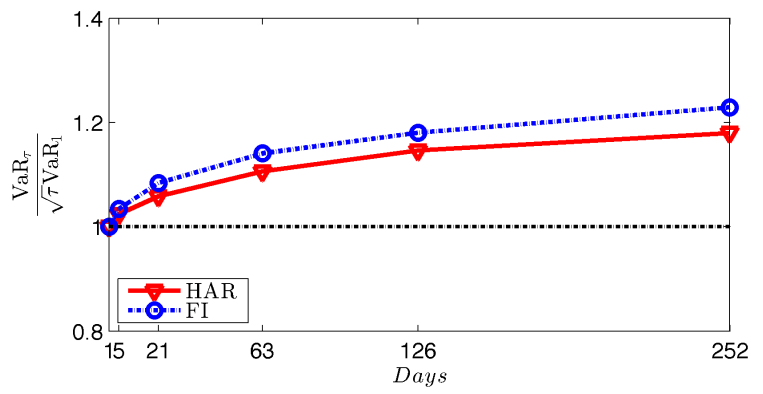

(b) $\alpha=1 \%$

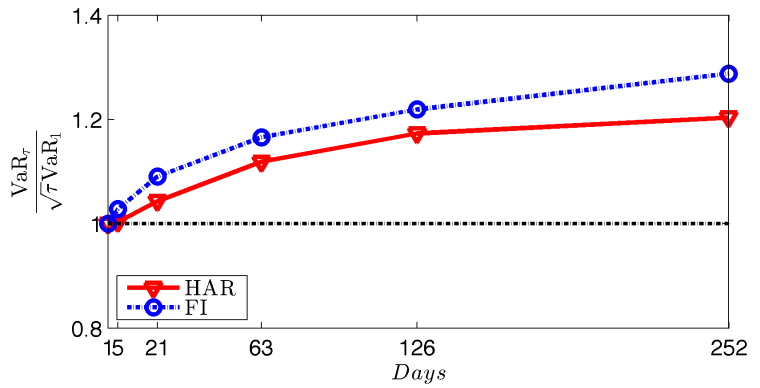

(c) $\alpha=0.5 \%$

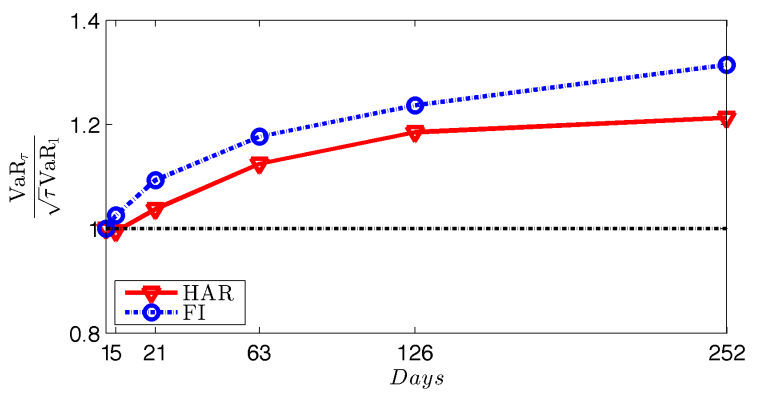

This graph shows the ratio between $\mathrm{VaR}_{\tau}$ obtained using different time series specifications for $\log$ Realized Variance and the long term VaR obtained using the square root of time rule for the $S P Y$. Moreover, panel A shows the ratio at the confidence level of $5 \%$, panel B displays the same ratio at $1 \%$ and panel $\mathrm{C}$ at $0.5 \%$. 
were to extend the investment horizon beyond one year we would observe that differences between the time series models would be more emphasized.

Using (4.10) we can estimate the scaling factor, $\xi$, implied by the mixture model and compare it to other scaling laws. We can estimate the scaling factor using the following regression:

$$
\ln \mathrm{VaR}_{\tau}=c+\xi \ln \tau+u_{\tau}
$$

where the regressand is obtained from the different dynamic models.

Table 4.6: Estimates of the scaling parameter

\begin{tabular}{|c|c|c|c|c|c|c|}
\hline \multirow[t]{2}{*}{ Par. } & \multicolumn{3}{|c|}{ HAR } & \multicolumn{3}{|c|}{ FI } \\
\hline & $\alpha=5 \%$ & $\alpha=1 \%$ & $\alpha=0.5 \%$ & $\alpha=5 \%$ & $\alpha=1 \%$ & $\alpha=0.5 \%$ \\
\hline$c$ & 0.2599 & 0.6427 & 0.7602 & 0.2620 & 0.6520 & 0.7732 \\
\hline$\xi$ & 0.5404 & 0.5527 & 0.5579 & 0.5470 & 0.5611 & 0.5669 \\
\hline
\end{tabular}

This table shows the estimates of regression in (4.11) for the two models and three confidence levels. The columns refer to the dynamic models and to the confidence level employed to compute VaR.

Table 4.6 displays the estimates of above mentioned regression. The regression results show that for both models and all confidence level we should scale more than square root of time. In fact, the estimates of $\xi$ are all greater than 0.5. Consistent with fat-tail behavior we recognize that a more conservative scaling is obtained when considering higher risk level. Our implied scaling law differs from the one derived in the Extreme Value Theory framework. This states that if the tail of $r_{t}$ is sampled from a power law distribution with tail index $\delta$ then multi-period VaR is obtained by setting $\xi$ equal to the inverse of the tail index in (4.10). However, this approach differs from one based on volatility for two elements. The estimate of the resulting multiperiod VaR can be diverse because either the daily VaR is different (level effect) or the scaling is different (slope effect) as pointed out by Danielsson and De Vries (2000). To compare our findings with those in Danielsson and De Vries (2000), we estimate the tail index for the SPY and for the other companies in our dataset using the Hill method. As Danielsson and De Vries (2000), we find $\hat{\xi}^{\text {Hill }}$ between 0.34 and 0.25 . This means that, assuming the same estimate for the daily VaR, implies a $\tau$ periods VaR which is flatter than the what our models imply.

This subsection has analyzed the implications of the dynamic models on VaR when volatility is assumed to be at a median level, yet given that volatility can be at high or low levels, the next section investigates this aspect on VaR. 


\subsubsection{Volatility Levels}

Time varying volatility strongly contrasts the statement that is at the basis of any scaling law. This has a major role when we compute VaR not only for short horizons but for long ones as well. Volatility is in fact described by a strong persistent process and its effect last long through time. Figure 4.5 shows the time series of VaR computed at $\alpha=0.5 \%$ for both dynamic processes of $h_{t}$. The first thing to notice is that the HAR model presents a smoother time series compared to the Fractional. This is due to the fact that the HAR model has a memory restricted to 22 lags therefore encompassing the last month volatility information.

Figure 4.5: Time series of annual VaR for the SPY

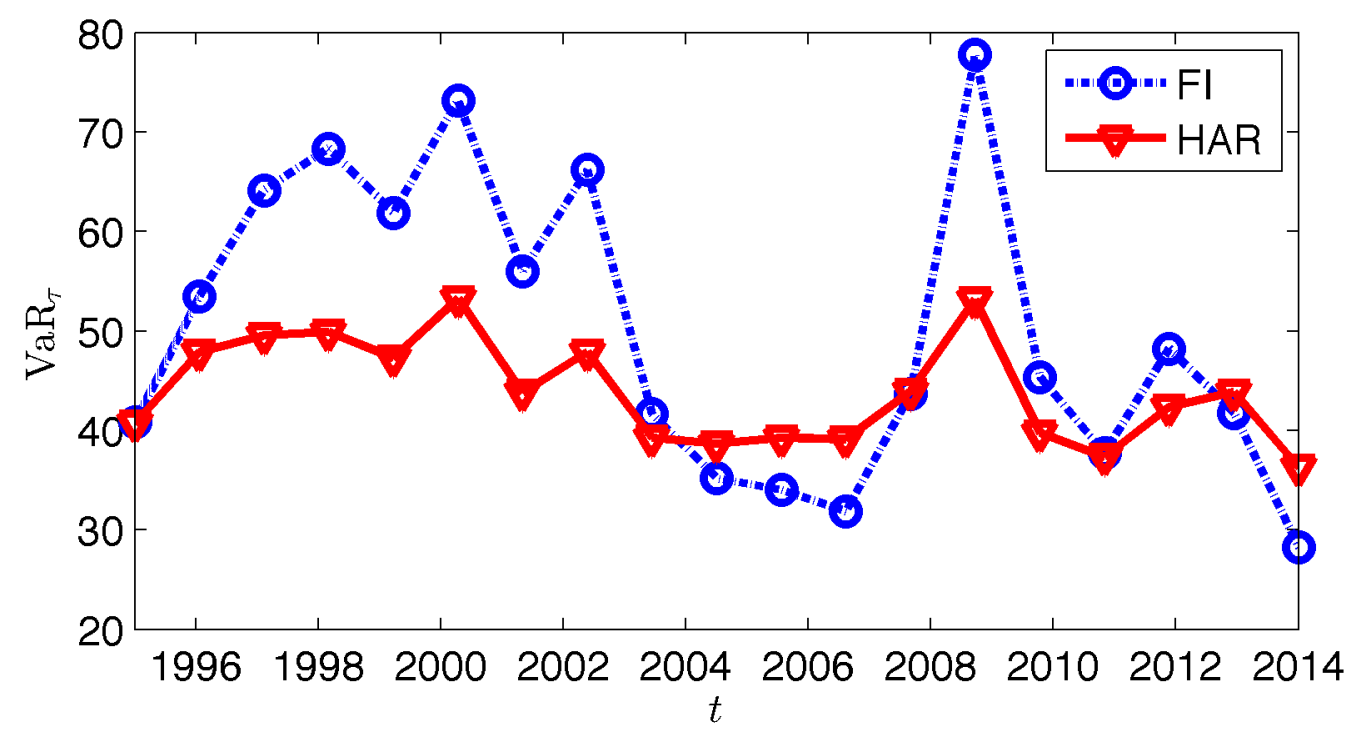

This graph shows the time series of VaR for the SPY for both dynamic processes of log variances. VaR is computed at $\alpha=0.5 \%$

In the previous subsection we have considered the case where volatility is at the median level. Now we consider two case: one where recent volatility is low and the other one where it is high. This allows to get more insights on how VaR aggregates under periods characterized by extreme market turmoil or in calm ones.

Figure 4.6 again shows the scaled VaR, but now at different levels of volatility. When volatility is high the Fractional model predicts a VaR which is still more conservative than square root of time. Instead, the HAR model implies a VaR that does not grow with the horizon. This is the result of the mean reversion of volatility combined with the increasing Vol-of-Vol. At this high volatility state, the two effects almost cancel. On the other hand, when volatility is low the HAR model exhibits a more conservative VaR than the Fractional. When volatility is high the HAR model reverts to the median much faster 
Figure 4.6: $\operatorname{VaR}_{\tau}$ at different levels of $\sigma_{t}^{2}$

(a) High Volatility

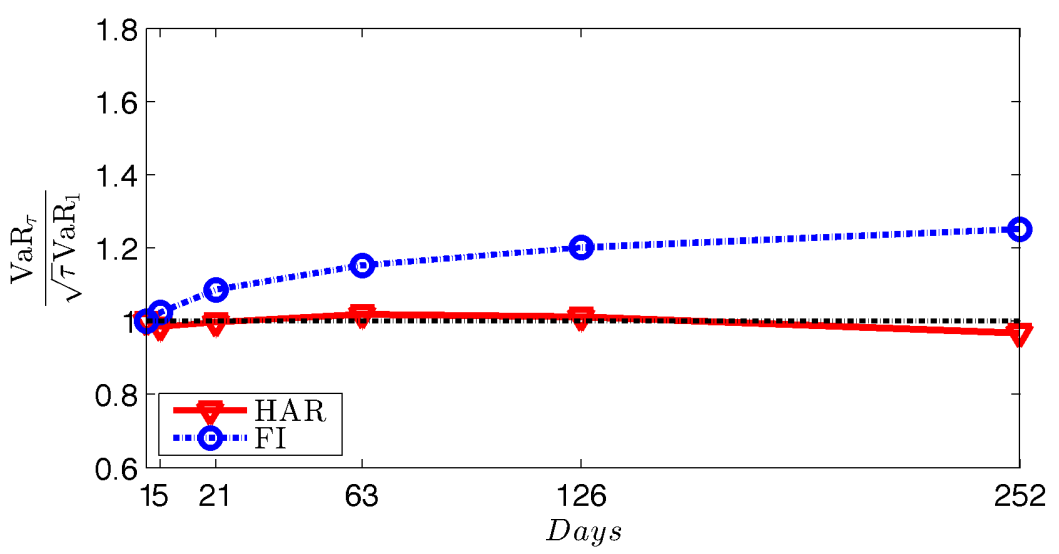

(b) Low Volatility

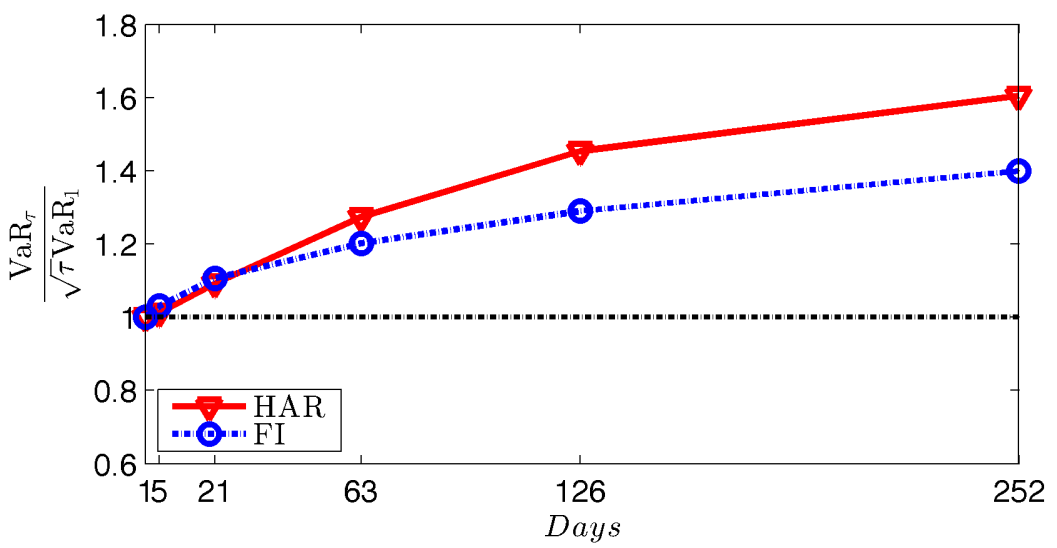

This graph shows the ratio between $\mathrm{VaR}_{\varnothing}$ obtained using two dynamic models for log Realized Variance and the multi-period VaR obtained using the square root of time rule for the SPY for two volatility levels. Panel A shows the ratio when volatility is high, panel B displays the same ratio when volatility is low. The high value of volatility is set equal to the seventy-fifth percentile of the unconditional volatility distribution, whereas the low one is set equal to the twenty-fitfth percentile. In both Panels, the confidence level is set to $\alpha=0.5 \%$. 
than the Fractional and hence the distribution of $S_{t+\tau}^{2}$ is less dispersed implying that the predictive density of returns is less fat-tailed.

\subsubsection{The Role of Persistence}

So far, we have focused on the aggregation properties for the $S P Y$, yet our dataset contains 28 companies which differ in the degree of persistence. Therefore, this section investigates the role of persistence in the aggregation of VaR. More specifically, we focus on the companies that present the weakest and strongest persistence, being Merck and Co. and Walmart respectively.

Figure 4.7: Ratio of $\mathrm{VaR}_{\tau}$ obtained with two different approaches

(a) Merck and Co

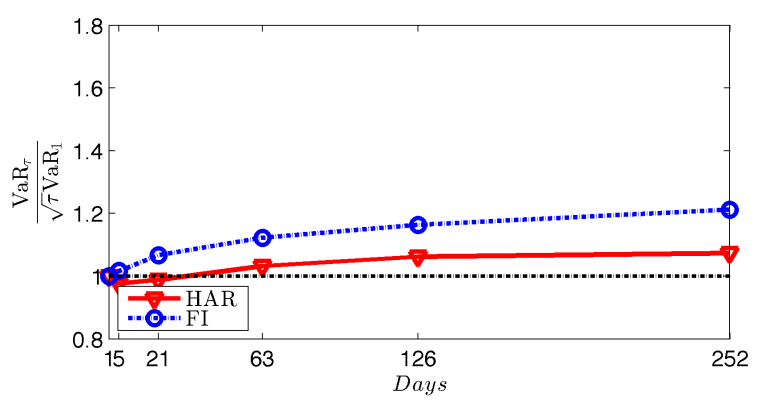

(b) Walmart

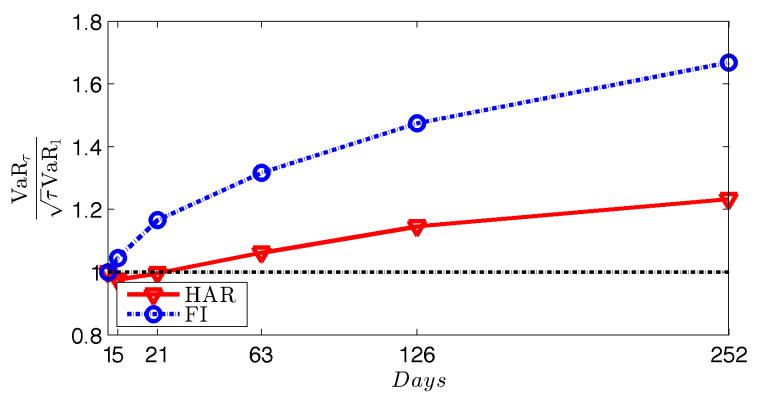

This graph shows the ratio between $\mathrm{VaR}_{\tau}$ obtained from the mixture model and using the square root of time rule for the least and most persistent stocks. VaR is computed at $\alpha=0.5 \%$.

For Merck and Co in Figure 4.7 we note that the Mixture model presents a more conservative VaR than the square root of time, this estimate is approximately $20 \%$ bigger for the Fractional model and slightly less than $10 \%$ for the HAR model. On the other hand for Walmart, the annual VaR is roughly $20 \%$ bigger for the HAR and $60 \%$ for the other specification. ${ }^{6}$

\subsubsection{An Approximate Scaling Law}

We have highlighted, that VaR aggregation depends on the volatility level and the degree of persistence of the volatility process. This section provides an approximate scaling law based on these two facts.

For each stock we consider the following transformation:

$$
x_{i, t, \tau}=\ln \operatorname{VaR}_{i, t, \tau}-\frac{1}{2} \ln \tau-\ln \operatorname{VaR}_{i, t, 1}
$$

\footnotetext{
${ }^{6}$ For the other confidence levels the results are available in the appendix in Figure 4.A.2. Qualitatively the interpretation of the results does not change compared to the case above mentioned. Moreover, we refer to table 4.A.3 in the Appendix for the actual values of $\mathrm{VaR}_{\tau}$.
} 
This expresses multi-period VaR in deviation from the square root of time rule for each company.

We have twenty years of log Realized Variances and to study annual VaR aggregation we are left with nineteen non overlapping observations of annual returns. For each company we consider the volatility level at the end of the year. We then have a vast number of multiperiod VaR that allows us to get a general pattern to construct a scaling law considering the current volatility state, the aggregation horizon and the persistence of the process.

More precisely recalling the transformation from equation (4.12) we run the following regression.

$$
x_{i, t, \tau}=b_{0}+b_{1} \ln \tau+b_{2} \lambda_{i}+b_{3} h_{i, t}+b_{4} \lambda_{i} \ln \tau+b_{5} \lambda_{i} h_{i, t}+b_{6} h_{i, t} \ln \tau+u_{i, t, \tau}
$$

where $\lambda_{i}$ is either $d_{i}$ for the Fractional model or $f_{i}$ for the HAR. Using this regression we can test four hypotheses. The first one is that annual VaR is a scaled version of the daily one. This implies that the all coefficients that show dependence on $h_{i, t}$ are zero. The second hypothesis refers to conditioning annual VaR on current volatility. Mean reversion implies that the higher the volatility the lower the scaling factor. The third hypothesis states that the higher the persistence the less it depends on current volatility. Finally, the more persistent a stock is the larger the scaling factor for annual VaR. Table 4.7 reports the estimates for the coefficients of this regression.

The first hypothesis is rejected as the parameters related to $h_{i, t}$ are statistically different from zero. The second hypothesis predicts that the partial derivative with respect to $\ln \sigma_{i, t}$ is negative. This happens for some $\tau$ for both model specifications. For example, considering the case at $\alpha=0.5 \%$ of Merck for the Fractional model we see that this partial derivative is positive for $\tau=5$ and negative for all the other values. Instead, for Walmart this is always negative. On the other hand, for the HAR model the reverse happens: in the high persistence case, this is positive for $\tau=5$ and negative for the other horizons. Instead, in the low persistence case this is always negative. The third hypothesis predicts that the interaction between persistence and volatility is negative. For the HAR model this is not the case, its scaling strongly positively depends on current value of volatility. For the Fractional model this is not the case. The fourth hypothesis is related to the interaction between persistence and horizon and predicts a positive coefficient. For both models we notice that this is confirmed.

Based on this regression we compute the scaling parameter for each company $i$, that depends on the current value of volatility and the persistence of the time series model.

$$
\frac{\partial \ln \operatorname{VaR}_{i, t, \tau}}{\partial \ln \tau}=\psi_{i, t}=\frac{1}{2}+b_{1}+b_{4} \lambda_{i}+b_{6} \ln \sigma_{i, t}
$$


Table 4.7: Estimates of scaling law regression

\begin{tabular}{|c|c|c|c|c|c|c|}
\hline \multirow[t]{2}{*}{ Par. } & \multicolumn{3}{|c|}{ HAR } & \multicolumn{3}{|c|}{ FI } \\
\hline & $\alpha=5 \%$ & $\alpha=1 \%$ & $\alpha=0.5 \%$ & $\alpha=5 \%$ & $\alpha=1 \%$ & $\alpha=0.5 \%$ \\
\hline \multirow[t]{2}{*}{ const } & 0.996 & 2.009 & 2.425 & 0.465 & 0.651 & 0.725 \\
\hline & $(0.512)$ & $(0.534)$ & $(0.541)$ & $(0.044)$ & $(0.047)$ & $(0.048)$ \\
\hline \multirow[t]{2}{*}{$\ln \tau$} & -0.269 & -0.706 & -0.886 & -0.140 & -0.278 & -0.334 \\
\hline & $(0.111)$ & $(0.115)$ & $(0.116)$ & $(0.015)$ & $(0.015)$ & $(0.016)$ \\
\hline \multirow[t]{2}{*}{$\lambda_{i}$} & -1.093 & -2.199 & -2.654 & -0.882 & -1.239 & -1.382 \\
\hline & $(0.537)$ & $(0.560)$ & $(0.567)$ & $(0.079)$ & $(0.083)$ & $(0.084)$ \\
\hline \multirow[t]{2}{*}{$h_{i, t}$} & -4.465 & -4.498 & -4.519 & 0.078 & 0.139 & 0.163 \\
\hline & $(0.252)$ & $(0.258)$ & $(0.267)$ & $(0.065)$ & $(0.068)$ & $(0.069)$ \\
\hline \multirow[t]{2}{*}{$\lambda_{i} \ln \tau$} & 0.336 & 0.803 & 0.996 & 0.367 & 0.623 & 0.726 \\
\hline & $(0.115)$ & $(0.120)$ & $(0.122)$ & $(0.026)$ & $(0.026)$ & $(0.027)$ \\
\hline \multirow[t]{2}{*}{$\lambda_{i} h_{i, t}$} & 4.818 & 4.859 & 4.883 & 0.045 & -0.045 & -0.080 \\
\hline & $(0.261)$ & $(0.265)$ & $(0.274)$ & $(0.111)$ & $(0.116)$ & $(0.119)$ \\
\hline \multirow[t]{2}{*}{$\ln \tau h_{i, t}$} & -0.108 & -0.111 & -0.112 & -0.071 & -0.072 & -0.072 \\
\hline & $(0.004)$ & $(0.005)$ & $(0.005)$ & $(0.005)$ & $(0.005)$ & $(0.005)$ \\
\hline
\end{tabular}

This table shows the estimates of the coefficient and standard errors clustered at company level of regression in (4.13) for the different models we consider at three confidence levels.

Based on (4.14) we can obtain a scaling law for companies that are characterized by a certain level of persistence and are currently in a high or low volatility state. This way we create a map according to persistence and volatility state.

The companies are divided in three categories: low, medium and high persistence. The cut-off is set such that we have approximately the same number of companies for each category. The same is applied to the volatility states for each company.

Based on this we create the map as in Table 4.8 for $\alpha=0.5 \%$ : this is composed by 9 squares and each square contains the average of the scaling law for each company and every state and denote it by $\bar{\psi}_{i, t}$. Each part of the table denotes an approximate scaling for the company characterized by that persistence and for that specific volatility state. Considering the upper right square, meaning high volatility and low persistence, we see that the scaling is closer to the value of 0.5 compared to the other extreme case in the lower left corner.

The other interesting element in the table is $\overline{\bar{\psi}}$ which is an approximate unconditional scaling law. For each single element of the inner table we can infer whether the scaling is statistically different from the square root of time rule. For each element in this table we reject square root of time rule hypothesis except for the HAR in the HIGH-HIGH case. Rejection of this hypothesis also applies for the scalings at other confidence levels, which are reported in table 4.A.4 and 4.A.5 in the Appendix. 
Table 4.8: Scalings for both models for $\alpha=0.5 \%$

\begin{tabular}{lccc|c|cccc}
\hline \multicolumn{4}{c}{ HAR } & \multicolumn{5}{c}{ FI } \\
\hline \multicolumn{3}{c}{$h_{i t}$} \\
\hline$\lambda_{i}$ & LOW & MEDIUM & HIGH & ALL & LOW & MEDIUM & HIGH & ALL \\
\hline LOW & 0.612 & 0.559 & 0.510 & 0.560 & 0.614 & 0.581 & 0.550 & 0.582 \\
MEDIUM & 0.632 & 0.579 & 0.521 & 0.577 & 0.631 & 0.595 & 0.552 & 0.593 \\
HIGH & 0.633 & 0.569 & 0.504 & 0.569 & 0.663 & 0.622 & 0.584 & 0.623 \\
\hline ALL & 0.626 & 0.569 & 0.511 & 0.568 & 0.636 & 0.599 & 0.562 & 0.599 \\
\hline
\end{tabular}

This table contains the scaling for the two time series specifications at $\alpha=0.5 \%$. Moreover, the fourth and the last column of the table report the averages for a given persistence group across the different volatility states. On the other hand the last row, in column one to three and five to seven, reports the averages across the different persistence classes for a given volatility state. The last remaining elements are the ones corresponding to the positions ALL-ALL: this is the average scaling law $\overline{\bar{\psi}}$ for the two different models

\subsubsection{Alternative methods and Backtesting}

This section introduces first other methods used to compute long term VaR. Second we assess the quality of the models employed to compute annual VaR. Embrechts, Kaufmann, and Patie (2005) study a relatively large set of models in order to compute annual VaR. Among the ones they have adopted, we compare the mixture model and our approximate scaling to the EVT based annual VaR and the one obtained using a random walk for log prices based on a lower frequency. Under the first approach multi-period VaR is obtained as follows:

$$
\operatorname{VaR}_{\tau}^{\mathrm{EVT}}(\alpha)=\tau^{\widehat{\xi}} \operatorname{VaR}_{1}(\alpha)
$$

where

$$
\operatorname{VaR}_{1}(\alpha)=X_{K}\left(\frac{K / T}{\alpha}\right)^{\widehat{\xi}}
$$

is the one period VaR. The second model we adopt is the square root of time on a white noise on a lower frequency. Let us denote by $\tau_{H}$ the number of observations at a frequency higher than the annual one denoted $\tau_{A}$. Then, annual VaR is obtained as follows:

$$
\operatorname{VaR}^{\mathrm{RW}}(\alpha)=m_{\tau_{H}}\left(\frac{\tau_{A}}{\tau_{H}}\right)-s_{\tau_{H}}\left(\frac{\tau_{A}}{\tau_{H}}\right)^{\frac{1}{2}} Q(\alpha)
$$


where $m_{\tau_{H}}$ is the mean of returns, $s_{\tau_{H}}$ is the standard deviation of returns at a higher frequency than annual and $Q(\alpha)$ denotes the quantile of the Normal distribution at the confidence level $\alpha$.

For long horizons such as one year assessing the goodness of a risk model is difficult. This is due to low number of non-overlapping observations to backtest the risk model. In fact, if $T$ is the length of the sample at the daily frequency the number of non overlapping observations at a lower frequency reduces it is approximately $\frac{T}{\tau_{A}}$. In our case we are left with $M=19$ non-overlapping observations. As a partial solution we pool the observations coming from the cross section of companies in our dataset. This way we have a total of $M \times N$ observations to backtest the different strategies. Embrechts, Kaufmann, and Patie (2005) backtest the competing models by aggregating similar asset classes.

Common practice in risk management is to assess the performance a risk model by counting the number of $\mathrm{VaR}$ violations, $I_{t}$. If the fraction of violation is not far from the confidence level $\alpha$ we have set, then we can conclude that model performs well. Nieto and Ruiz (2016) review different backtesting techniques starting from the one developed by Kupiec. This states that the expected value of $I_{t}$ should be close to the confidence level at which VaR is computed. This can be implemented by means of a simple regression of the violations on a constant.

$$
I_{i, t}=c+\epsilon_{i, t}
$$

We obtain an estimate of $c$ that gives the percentage of violations of one year VaR. The null hypothesis for this testing problem states that $c=\alpha$. Table 4.9 reports the estimate of $c$ and the standard error computed by clustering at stocks level.

First, this table highlights that the Mixture model provides a satisfactory risk assessment when compared to other strategies. For example, although we cannot formally reject it due to the low power of the test, using the square root of time rule leads to vastly underestimating annual VaR at each confidence level. The mixture model slightly overestimates risk at $\alpha=5 \%$ and it underestimates it at the higher confidence levels. Interesting to notice is the performance of the approximate scaling law: at $5 \%$ and $1 \%$ it also overestimates risk and for the highest risk level it is very close to the required confidence level of $0.5 \%$. The fact that the Fractional model provides fewer violations follows from the arguments of the previous sections. Using the models that Embrechts, Kaufmann, and Patie (2005) study we reach the following conclusions: first, EVT strongly underestimates annual VaR when calibrated on daily frequencies for any confidence level considered. Second, following the advice in Embrechts, Kaufmann, and Patie (2005) we calibrate the white noise on monthly returns and then scale to annual using (4.17). We 
Table 4.9: Performance of annual VaR measures

\begin{tabular}{|c|c|c|c|c|c|c|c|c|}
\hline & HAR & $\tau^{0.5} \mathrm{HAR}$ & $\tau^{\overline{\bar{\psi}}} \mathrm{HAR}$ & FI & $\tau^{0.5} \mathrm{FI}$ & $\tau^{\overline{\bar{\psi}}} \mathrm{FI}$ & EVT & RW \\
\hline \multicolumn{9}{|c|}{$\alpha=5 \%$} \\
\hline $\mathrm{c}$ & 0.047 & 0.070 & 0.045 & 0.039 & 0.064 & 0.034 & 0.156 & 0.055 \\
\hline $\mathrm{SE}(\mathrm{c})$ & 0.029 & 0.035 & 0.028 & 0.028 & 0.031 & 0.022 & 0.057 & 0.028 \\
\hline t-stat & -0.104 & 0.555 & -0.172 & -0.371 & 0.442 & -0.721 & 1.854 & 0.159 \\
\hline \multicolumn{9}{|c|}{$\alpha=1 \%$} \\
\hline $\mathrm{c}$ & 0.015 & 0.038 & 0.017 & 0.013 & 0.038 & 0.009 & 0.100 & 0.019 \\
\hline $\mathrm{SE}(\mathrm{c})$ & 0.011 & 0.025 & 0.013 & 0.011 & 0.025 & 0.006 & 0.042 & 0.012 \\
\hline t-stat & 0.443 & 1.126 & 0.524 & 0.279 & 1.126 & -0.100 & 2.111 & 0.732 \\
\hline \multicolumn{9}{|c|}{$\alpha=0.5 \%$} \\
\hline c & 0.013 & 0.028 & 0.008 & 0.011 & 0.030 & 0.006 & 0.068 & 0.015 \\
\hline $\mathrm{SE}(\mathrm{c})$ & 0.010 & 0.019 & 0.006 & 0.009 & 0.019 & 0.006 & 0.031 & 0.009 \\
\hline t-stat & 0.855 & 1.231 & 0.431 & 0.662 & 1.335 & 0.113 & 2.006 & 1.144 \\
\hline
\end{tabular}

Performance of the different methods to compute annual VaR. The robust standard errors are computed clustering at the companies level. The first and fourth column refer to the performance of VaR obtained using the mixture model for the two time series models. The second and fifth column refer to the square root of time rule applied to daily VaR obtained from the mixture model for the two time series specifications. The third and sixth column refer to the approximate scaling law applied to daily VaR obtained from the mixture model for the two time series specifications. The last two column refer to annual VaR obtained using EVT and the Random Walk, respectively. 
confirm their findings: at lower risk levels the performance of this strategy is satisfactory whereas for the others the degree of underestimation worsens.

\subsection{Conclusions}

We study temporal aggregation of VaR using a Mixture model calibrated on Realized Variances using high frequency data. Based on the performance of this model, we find that aggregation of VaR from a daily to an annual horizons requires a scaling factor of order higher than 0.5. We obtain this scaling factor focusing on two empirical features: the current level of volatility and its persistence.

First, by considering the current volatility level this scaling law takes into account the more recent market information. Second, persistence in volatility implies that if the current market risk will last long in time therefore having relevant implications for the annual VaR. Using two time series specifications we show that a model with longer memory results in a scaling law that is more conservative compared to other existing approaches and that varies according to the recent market status.

This work has focused on estimating annual VaR for equity portfolios. Yet, financial institutions hold portfolios composed by a multitude of asset classes. Pension funds for instance have a significant exposure to long term government bonds. When it comes to delivering an annual VaR estimate a further complication arises when the VaR of the single asset classes must be aggregated. A key element in the aggregation of the single VaR is the probability of joint extreme returns. In our setting we could do this by modeling the realized covariance structure between stocks and bonds. Last, many risk management related issues are naturally multivariate. It would be interesting to assess how modeling covariances affect the tail of the multiperiod return of a portfolio. 


\section{Appendix}

\section{Monte Carlo Simulation}

\section{AR models}

Any $\mathrm{AR}(\mathrm{p})$ model can be written in state space form as follows:

$$
Y_{t+1}=F Y_{t}+G E_{t+1}
$$

where $Y_{t+1}$ is a vector containing $h_{t+1}$ in the first position and its lags until $h_{t-p+2}$ where $p=22$ is the order of the restricted autoregressive process. Moreover, $\mathrm{F}$ is the matrix containing in the first row the autoregressive coefficients: for the HAR model it contains the coefficient estimates of the transformed $\mathrm{AR}(22)$ process. The lower $p-1 \times p-1$ block contains an identity matrix and the last column is composed by $p-1$ zeros. Finally $G$, is a column vector containing a one in the first position and zeros elsewhere. By simple recursion,

$$
\begin{aligned}
\mathbb{E}\left[Y_{t+T}\right] & =F^{T} Y_{t} \\
\mathbb{V}\left[Y_{t+T}\right] & =\omega^{2} \sum_{j=0}^{T-1} F^{j} G G^{\prime}\left(F^{j}\right)^{\prime}
\end{aligned}
$$

\section{FI Model}

Recalling the FI model as in equation :

$$
(1-L)^{d}\left(h_{t}-c\right)=\omega \eta_{t+1}
$$

Define $y_{t}=h_{t}-c$. Simulations of $y_{t+T}$ is performed using the recursive structure of the coefficients of the Fractional model:

$$
y_{t+T}=\sum_{s=1}^{T} b_{s} y_{t+T-s}+\omega \eta_{t+T}
$$

where

$$
b_{s+1}=b_{s} \frac{s-d}{s+1}
$$

with $b_{0}=1$. 


\section{Additional Tables}

Table 4.A.1: Moments of Integrated Variance for two companies

\begin{tabular}{|c|c|c|c|c|}
\hline \multirow[t]{2}{*}{$\tau$} & \multicolumn{2}{|c|}{ HAR } & \multicolumn{2}{|c|}{ FI } \\
\hline & $\mathbb{E}\left(S_{t+\tau}^{2} \mid m\right) / \tau$ & $\mathbb{V}\left(S_{t+\tau}^{2} \mid m\right) / \tau$ & $\mathbb{E}\left(S_{t+\tau}^{2} \mid m\right) / \tau$ & $\mathbb{V}\left(S_{t+\tau}^{2} \mid m\right) / \tau$ \\
\hline \multicolumn{5}{|c|}{ Panel A: Least Persistent Stock } \\
\hline 1 & 1.711 & 0.933 & 1.716 & 0.950 \\
\hline 5 & 1.754 & 0.699 & 1.821 & 0.918 \\
\hline 21 & 1.820 & 0.690 & 1.975 & 1.060 \\
\hline 63 & 1.923 & 0.860 & 2.139 & 1.237 \\
\hline 126 & 2.004 & 0.959 & 2.265 & 1.378 \\
\hline 252 & 2.070 & 0.941 & 2.414 & 1.553 \\
\hline \multicolumn{5}{|c|}{ Panel B: Most Persistent Stock } \\
\hline 1 & 1.379 & 0.686 & 1.392 & 0.725 \\
\hline 5 & 1.408 & 0.507 & 1.507 & 0.819 \\
\hline 21 & 1.465 & 0.538 & 1.742 & 1.203 \\
\hline 63 & 1.568 & 0.758 & 2.053 & 1.771 \\
\hline 126 & 1.714 & 1.057 & 2.411 & 2.511 \\
\hline 252 & 1.878 & 1.363 & 2.885 & 3.646 \\
\hline
\end{tabular}

This table shows the scaled moments of the Integrated Variance distribution simulated for the two time series specifications. Panel A shows the scaled moments for Merck and Co. Panel B shows the scaled moments for Walmart. The different rows display the moments as a function of $\tau$. 
Table 4.A.2: VaR aggregation in different volatility periods

\begin{tabular}{|c|c|c|c|c|c|c|}
\hline \multirow[t]{2}{*}{$\bar{\tau}$} & \multicolumn{3}{|c|}{ HAR } & \multicolumn{3}{|c|}{ FI } \\
\hline & $\alpha=5 \%$ & $\alpha=1 \%$ & $\alpha=0.5 \%$ & $\alpha=5 \%$ & $\alpha=1 \%$ & $\alpha=0.5 \%$ \\
\hline \multicolumn{7}{|c|}{ Panel A: High Volatility Period } \\
\hline 1 & 1.901 & 2.901 & 3.317 & 1.925 & 2.939 & 3.359 \\
\hline 5 & 4.303 & 6.435 & 7.299 & 4.439 & 6.738 & 7.689 \\
\hline 21 & 8.861 & 13.337 & 15.164 & 9.477 & 14.570 & 16.717 \\
\hline 63 & 15.201 & 23.372 & 26.816 & 17.077 & 26.626 & 30.735 \\
\hline 126 & 21.001 & 32.651 & 37.666 & 24.771 & 39.099 & 45.282 \\
\hline 252 & 28.668 & 44.289 & 50.933 & 35.791 & 57.217 & 66.733 \\
\hline \multicolumn{7}{|c|}{ Panel B: Low Volatility Period } \\
\hline 1 & 0.945 & 1.442 & 1.647 & 0.933 & 1.424 & 1.628 \\
\hline 5 & 2.193 & 3.285 & 3.728 & 2.167 & 3.285 & 3.750 \\
\hline 21 & 4.807 & 7.251 & 8.244 & 4.682 & 7.205 & 8.256 \\
\hline 63 & 9.334 & 14.465 & 16.650 & 8.622 & 13.467 & 15.534 \\
\hline 126 & 14.704 & 23.188 & 26.883 & 12.819 & 20.322 & 23.565 \\
\hline 252 & 23.162 & 36.269 & 41.955 & 19.332 & 30.943 & 36.149 \\
\hline
\end{tabular}

This table shows the VaR at three confidence levels computed for the different models. Panel A shows when the estimates when past volatility is assumed to be high whereas panel B when it is low The different rows display the aggregation horizon ranging from one day, first row, to one year, last row. 
Table 4.A.3: VaR aggregation for two companies

\begin{tabular}{|c|c|c|c|c|c|c|}
\hline \multirow[t]{2}{*}{$\tau$} & \multicolumn{3}{|c|}{ HAR } & \multicolumn{3}{|c|}{ FI } \\
\hline & $\alpha=5 \%$ & $\alpha=1 \%$ & $\alpha=0.5 \%$ & $\alpha=5 \%$ & $\alpha=1 \%$ & $\alpha=0.5 \%$ \\
\hline \multicolumn{7}{|c|}{ Panel A: Merck and Co. } \\
\hline 1 & 2.132 & 3.251 & 3.716 & 2.135 & 3.261 & 3.729 \\
\hline 5 & 4.847 & 7.172 & 8.102 & 4.922 & 7.439 & 8.475 \\
\hline 21 & 10.128 & 14.924 & 16.831 & 10.494 & 15.963 & 18.236 \\
\hline 63 & 17.980 & 26.863 & 30.449 & 18.883 & 28.977 & 33.229 \\
\hline 126 & 25.940 & 38.985 & 44.302 & 27.448 & 42.379 & 48.713 \\
\hline 252 & 37.347 & 55.841 & 63.322 & 40.040 & 62.245 & 71.761 \\
\hline \multicolumn{7}{|c|}{ Panel B: Walmart } \\
\hline 1 & 1.917 & 2.894 & 3.294 & 1.925 & 2.921 & 3.330 \\
\hline 5 & 4.347 & 6.387 & 7.189 & 4.470 & 6.812 & 7.786 \\
\hline 21 & 9.089 & 13.360 & 15.049 & 9.786 & 15.369 & 17.790 \\
\hline 63 & 16.222 & 24.417 & 27.766 & 18.241 & 29.642 & 34.796 \\
\hline 126 & 23.866 & 36.836 & 42.379 & 27.637 & 46.375 & 55.122 \\
\hline 252 & 35.132 & 55.481 & 64.459 & 42.195 & 73.197 & 88.219 \\
\hline
\end{tabular}

This table shows the VaR at three confidence levels computed for the different models for the least and most persistent stock. The different rows display the aggregation horizon ranging from one day, first row, to one year, last row.

Table 4.A.4: Scaling factor: $\alpha=1 \%$

\begin{tabular}{lccc|c|cccc}
\hline \multicolumn{3}{c}{ HAR } & \multicolumn{5}{c}{ FI } \\
\hline \multicolumn{3}{c}{$h_{i t}$} \\
\hline$\lambda_{i}$ & LOW & MEDIUM & HIGH & ALL & LOW & MEDIUM & HIGH & ALL \\
\hline LOW & 0.608 & 0.556 & 0.507 & 0.557 & 0.610 & 0.578 & 0.547 & 0.579 \\
MEDIUM & 0.626 & 0.573 & 0.516 & 0.572 & 0.625 & 0.589 & 0.547 & 0.587 \\
HIGH & 0.626 & 0.563 & 0.498 & 0.562 & 0.653 & 0.613 & 0.574 & 0.613 \\
\hline ALL & 0.620 & 0.564 & 0.507 & 0.564 & 0.630 & 0.593 & 0.556 & 0.593 \\
\hline
\end{tabular}

This table contains the scaling factor for the two time series specifications at $\alpha=1 \%$. Moreover, the fourth and the last column of the table report the averages for a given persistence group across the different volatility states. On the other hand the last row, in column one to three and five to seven, reports the averages across the different persistence classes for a given volatility state. The last remaining elements are the ones corresponding to the positions ALL-ALL: this is the average scaling law $\overline{\bar{\psi}}$ for the two different models 
Table 4.A.5: Scaling factor: $\alpha=5 \%$

\begin{tabular}{lccc|c|ccc|c}
\hline \multicolumn{4}{c}{ HAR } & \multicolumn{3}{c}{ FI } \\
\hline \multicolumn{3}{c}{$h_{i t}$} \\
\hline$\lambda_{i}$ & LOW & MEDIUM & HIGH & ALL & LOW & MEDIUM & HIGH & ALL \\
\hline LOW & 0.599 & 0.549 & 0.501 & 0.550 & 0.602 & 0.570 & 0.540 & 0.571 \\
MEDIUM & 0.612 & 0.561 & 0.505 & 0.559 & 0.610 & 0.575 & 0.534 & 0.573 \\
HIGH & 0.610 & 0.548 & 0.485 & 0.547 & 0.629 & 0.589 & 0.552 & 0.590 \\
\hline ALL & 0.607 & 0.552 & 0.497 & 0.552 & 0.614 & 0.578 & 0.542 & 0.578 \\
\hline
\end{tabular}

This table contains the scaling factor for the two time series specifications at $\alpha=5 \%$. Moreover, the fourth and the last column of the table report the averages for a given persistence group across the different volatility states. On the other hand the last row, in column one to three and five to seven, reports the averages across the different persistence classes for a given volatility state. The last remaining elements are the ones corresponding to the positions ALL-ALL: this is the average scaling law $\overline{\bar{\psi}}$ for the two different models 


\section{Additional Figures}

Figure 4.A.1: Ratio of $\mathrm{VaR}_{\tau}$ as function of volatility level

(a) High Volatility $\alpha=5 \%$

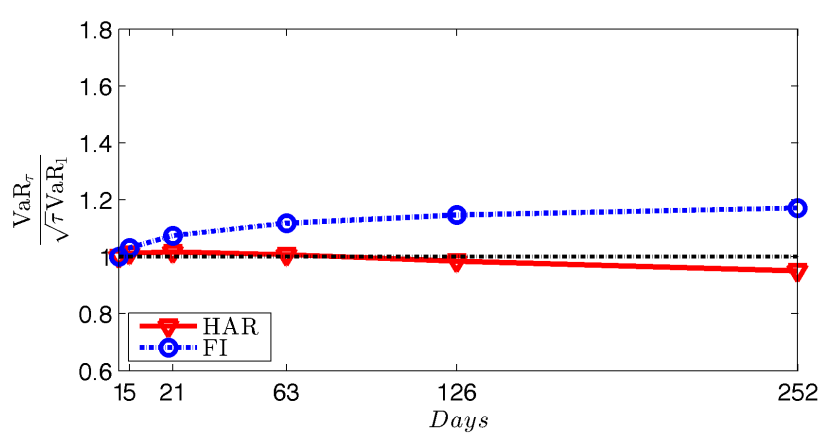

(c) Low Volatility $\alpha=5 \%$

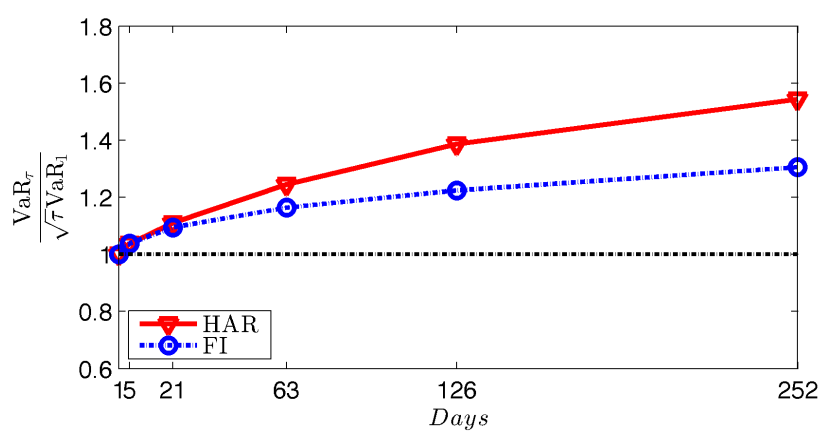

(b) High Volatility $\alpha=1 \%$

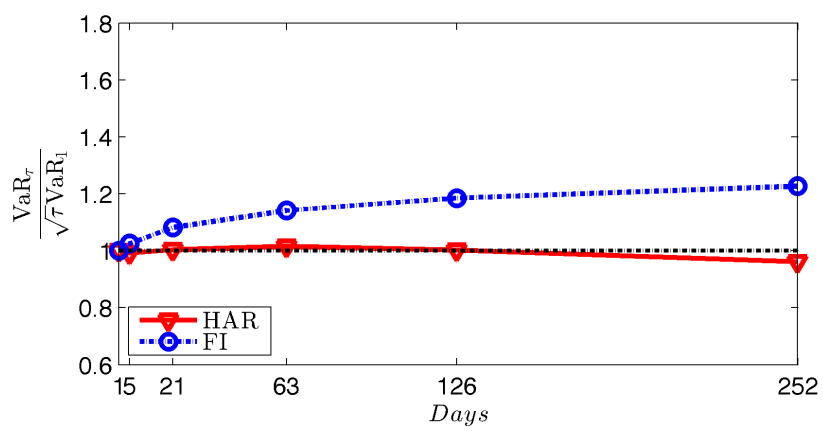

(d) Low Volatility $\alpha=1 \%$

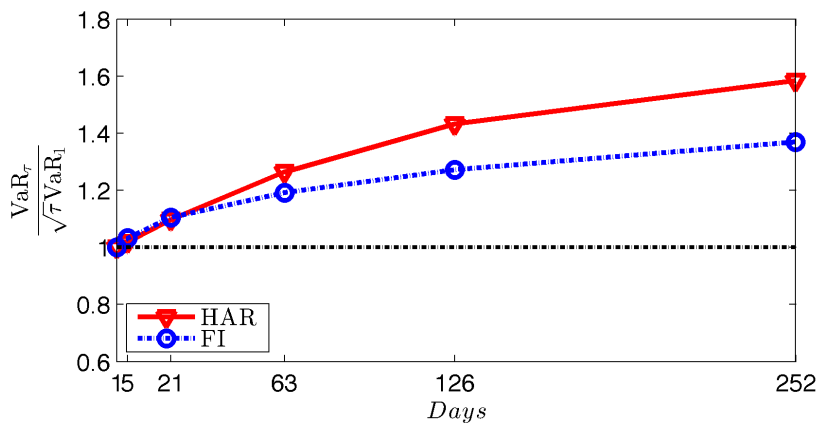

This graph shows the ratio between $\mathrm{VaR}_{\mathrm{T}}$ obtained using our model and different time series specifications for log Realized Variance and the long term VaR obtained using the square root of time rule for the SPY. Moreover, panel A shows the ratio at the confidence level of $5 \%$, panel $\mathrm{B}$ displays the same ratio at $1 \%$ when volatility is high. Also, panel $\mathrm{C}$ shows the ratio at the confidence level of $5 \%$, panel $\mathrm{D}$ displays the same ratio at $1 \%$ when volatility is low. 
Figure 4.A.2: Ratio of $\mathrm{VaR}_{\tau}$ as function of persistence

(a) Merck and Co $\alpha=5 \%$

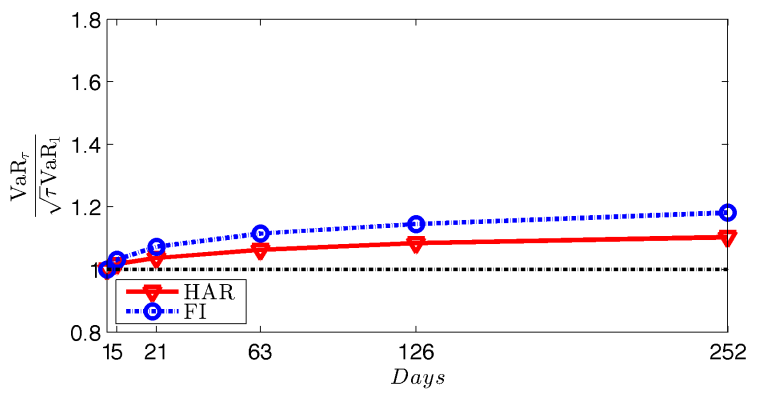

(c) Walmart $\alpha=5 \%$

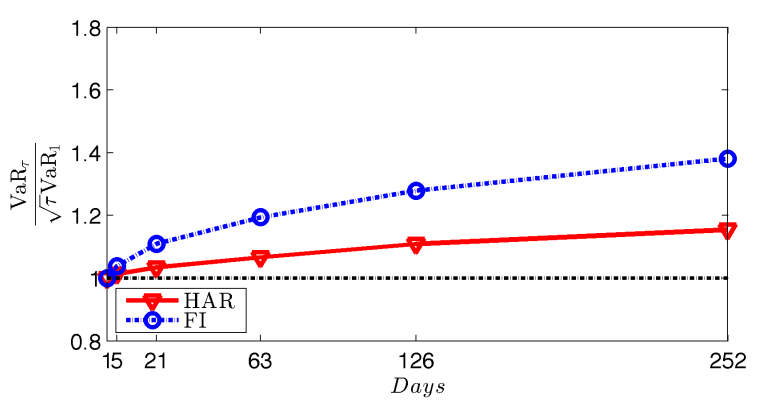

(b) Merck and $\operatorname{Co} \alpha=1 \%$

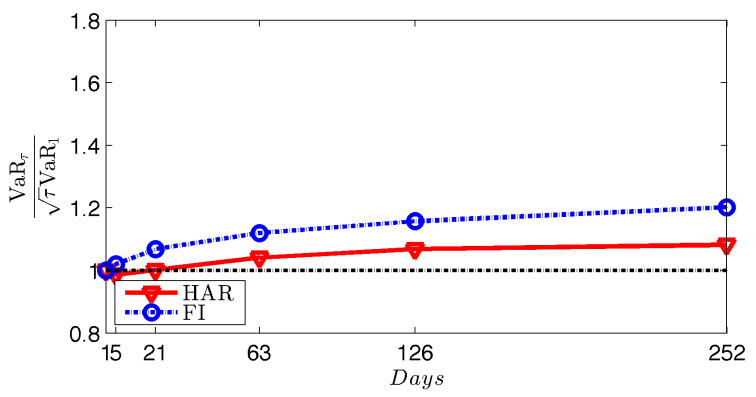

(d) Walmart $\alpha=1 \%$

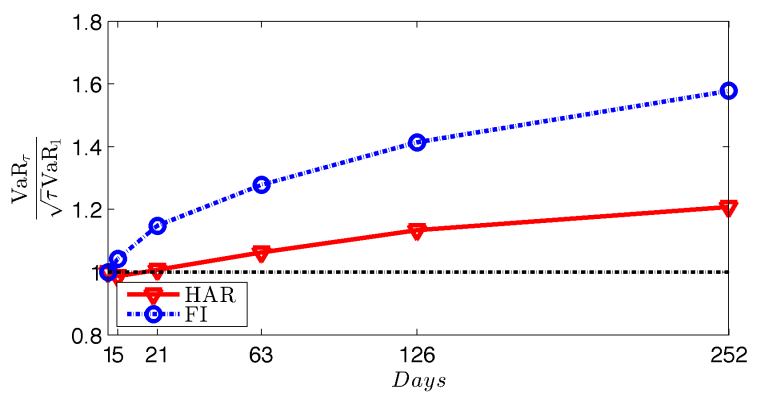

This graph shows the ratio between $\mathrm{VaR}_{\tau}$ obtained using our model and different time series specifications for log Realized Variance and the long term VaR obtained using the square root of time rule for the least persistent stock in panel a shows the ratio at the confidence level of $1 \%$ whereas panel b displays the same ratio at $5 \%$. The same ratio is shown in panel c for the most persistent stock at $5 \%$ and in panel $\mathrm{d}$ at $1 \%$. 


\section{Chapter 5}

\section{Conclusions}

Financial institutions make investment decisions to answer households needs. Yet, to protect investors' savings, regulators demand financial institutions to provide measures of riskiness of their business. More specifically, regulators require financial institutions to compute their economic capital based on measures that describe the potential loss under extreme negative circumstances. Supervisors give banks and pension funds the option to develop their own methods to compute the above mentioned capital requirement. Therefore the development of new methods to provide accurate risk measures is of interest for both practitioners and regulators. This thesis sheds light on some aspects related to equity risk and it provides answers to improve current risk management practice both from a practitioner and a regulatory standpoint.

For instance, chapter 2 deepens current knowledge on equity risk by investigating the characteristics of a stock index and its constituents. First, this chapter provides evidence that single stocks are characterized by parameters estimate that are very heterogeneous when compared to the index. Second, this chapter investigates the occurrence of big negative return in the index and how these relate to the single stocks. In the literature, these events are commonly denoted jumps. By jointly investigating the dynamics of jumps in the index and in the single stocks, chapter 2 highlights that a jump in the index is often driven by a large number of companies also experiencing a large negative return when macroeconomic news are released.

Chapter 3 and 4 provide new directions about risk modeling for financial institution that are characterized by a short or a long investment horizon. The availability of intra-day data allowed researchers to infer the properties of financial return more accurately and to measure well-defined theoretical objects more precisely: an example of this is given by volatility. The fact that volatility clusters in time is a useful property for financial decision making and risk management. Therefore, chapter 3 focuses on forecasting daily VaR using High-Frequency econometrics techniques. More specifically, the approach starts from 


\section{CONCLUSIONS}

an empirical stylized fact: daily return are approximately Gaussian when standardized by volatility estimated using High-Frequency data. This is not the case in a conditional volatility model where standardized return still exhibit the kurtosis of a fat-tailed random variable. In the setting I consider, volatility is characterized by its own stochastic process, therefore 3 investigates what is a reasonable density function of volatility for daily VaR forecasting purposes. This chapter concludes that a reasonable model for VaR is given by modeling variances in logs as they are close to be normally distributed.

When it comes to financial institutions with a long investment horizon, such as pension funds and insurance companies, regulators demand that they report an annual risk measure. In this respect, EIOPA requires pension funds and insurance companies to compute the VaR with an annual horizon to calculate their capital buffers. Yet, when such a long horizon is considered the number of non-overlapping observations diminishes very quickly therefore making inference about the tail of return distribution very difficult. EIOPA gives three options to pension funds and insurance companies: i) adopt the standard model, ii) partially adopt the standard model and develop their own and iii) use an own developed one only. In the first case, the requirement for market risk can be either computed using an aggregation formula or, if this approach is not applicable, adopt a prudent estimate of 40\%. Chapter 4 investigates this issue borrowing techniques from High-Frequency econometrics. More specifically, building on the findings in chapter 3, I show that, also for an investment horizon of a year, VaR should be computed using the most recent market conditions reflected in the volatility level. Periods of market turmoil are characterized by a high volatility and this level will long persist in time therefore having an impact on annual VaR.

Another method to compute multi-period VaR is given by the usage of a scaling law, a relation that links the high-frequency VaR to the lower frequency one. Its usage is appealing as it allows to obviating the problem of data reduction at long horizons. Yet, the derivation of a scaling law depends on the assumptions made for the return data generating process observed at a high frequency. For instance, the well-known square root of time rule is derived under very strong assumptions: in this case, the annual VaR is obtained by multiplying the daily one by the square root of the number of days in a year. Chapter 4 also provides an answer to this question by showing that the in the proposed model the scaling factor is higher than square root. The validity of this approach compared to the other scaling law is proven in the model validation part. 


\section{References}

Ait-Sahalia, Y, 1996, Testing continuous-time models of the spot interest rate, Review of Financial Studies 9, 385-426.

Andersen, Torben G, Tim Bollerslev, and Francis X Diebold, 2005, Roughing It Up: Including Jump Components, Review of Economics and Statistics 89, 701-720.

Andersen, Torben G., Tim Bollerslev, Francis X. Diebold, and Heiko Ebens, 2001, The distribution of realized stock return volatility, Journal of Financial Economics 61, 4376.

Andersen, Torben G., Tim Bollerslev, Francis X. Diebold, and Paul Labys, 2001, The Distribution of Realized Exchange Rate Volatility, Journal of the American Statistical Association 96, 42-55.

Andersen, Torben G, Tim Bollerslev, Francis X Diebold, and Paul Labys, 2003, Modeling and Forecasting Realized Volatility, Econometrica 71, 579-625.

Andersen, Torben G, Tim Bollerslev, Per Frederiksen, and Morten Orregard Nielsen, 2010, Continuous-Time Models, Realized Volatilities, and testable distributional implications for daily stock returns, Journal of Applied Econometrics 261, 233-261.

Asmussen, Søren, and Leonardo Rojas-nandayapa, 2008, Asymptotics of sums of lognormal random variables with Gaussian copula, Statistics and Probability Letters 78, 2709-2714.

Baillie, Richard T, 1996, Long memory processes and fractional integration in econometrics, Journal of Econometrics 73, 5-59.

Bakshi, G., C. Cao, and Z. Chen, 1997, Empirical Performance of Alternative Option Pricing Models, Journal of Finance 52, 2003-2049.

Bakshi, G., N. Kapadia, and D. Madan, 2003, Stock Return Characteristics, Skew Laws, and the Differential Pricing of Individual Equity Options, Review of Financial Studies $16,101-143$. 
Barndorff-Nielsen, O.E., and N. Shephard, 2004, Power and Bipower Variation with Stochastic Volatility and Jumps, Journal of Financial Econometrics 2, 1-48.

Barndorff-Nielsen, Ole E., 1978, Hyperbolic Distributions and Distributions on Hyperbolae, Scandinavian Journal of Statistics 5, 151-157.

Barndorff-Nielsen, Ole E., 1997, Normal Inverse Gaussian Distributions and Stochastic Volatility Modelling, Scandinavian Journal of Statistics 24, 1-13.

Barndorff-Nielsen, Ole E., and Neil Shephard, 2002a, Econometric analysis of realized volatility and its use in estimating stochastic volatility models, Journal of Royal Statistical Society B 64, 253-280.

Barndorff-Nielsen, Ole E., and Neil Shephard, 2002b, Estimating quadratic variation using realized variance, Journal of Applied Econometrics 17, 457-477.

Bates, D.S., 1996, Jumps and Stochastic Volatility: Exchange Rate Processes Implicit in Deutsche Mark Options, Review of Financial Studies 9, 69-107.

Bee, Marco, Debbie J Dupuis, and Luca Trapin, 2016, Realizing the extremes : Estimation of tail-risk measures from a high-frequency perspective, Journal of Empirical Finance $36,86-99$.

Beltratti, Andrea, and Claudio Morana, 2005, Statistical benefits of value-at-risk with long memory, Journal of Risk 7, 21-47.

Berkowitz, Jeremy, 2001, Testing density forecasts, with applications to risk management, Journal of Business 85 Economic Statistics 19, 465-474.

Berkowitz, Jeremy, Peter Christoffersen, and Denis Pelletier, 2011, Evaluating Value-atRisk Models with Desk-Level Data, Management Science 57, 2213-2227.

Bollen, N.P., and R.E. Whaley, 2004, Does Net Buying Pressure Affect the Shape of Implied Volatility Functions?, Journal of Finance 59, 711-753.

Bollerslev, Tim, 1986, Generalized Autoregressive Conditional Heteroskedasticity, Journal of Econometrics 31, 307-327.

Bollerslev, Tim, Andrew J Patton, and Rogier Quaedvlieg, 2016, Exploiting the Errors : A Simple Approach for Improved Volatility Forecasting, Journal of Econometrics 192, $1-18$.

Broadie, Mark, Mikhail Chernov, and Michael Johannes, 2007, Model specification and risk premia: Evidence from futures options, The Journal of Finance 62, 1453-1490. 
Caporin, Massimiliano, Aleksey Kolokolov, and Roberto Renó, 2017, Systemic Co-jumps, Journal of Financial Economics 126, 563-591.

Chourdakis, Kyriakos, and George Dotsis, 2011, Maximum Likelihood Estimation of NonAffine Volatility Processes, Journal of Empirical Finance 18, 533-545.

Christensen, Kim, Roel C A Oomen, and Mark Podolskij, 2014, Fact or friction: Jumps at ultra high frequency, Journal of Financial Economics 114, 576-599.

Christoffersen, Peter, 1998, Evaluating Interval Forecasts, International Economic Review $39,841-862$.

Christoffersen, P., F. Diebold, and T Schuermann, 1998, Horizon problems and extreme events in financial risk management, Reserve Bank NY Econ.Policy Rev Policy Rev, 109-118.

Christoffersen, Peter, and Kris Jacobs, 2004, The importance of the loss function in option valuation, Journal of Financial Economics 72, 291-318.

Christoffersen, P., K. Jacobs, and K. Mimouni, 2010, Volatility Dynamics for the S\&P500: Evidence from Realized Volatility, Daily Returns, and Option Prices, Review of Financial Studies 23, 3141-3189.

Christoffersen, Peter F, and Francis X Diebold, 2000, How Relevant is Volatility Forecasting for Financial Risk Management ?, Review of Economics and Statistics 82, 12-22.

Conley, T. G., L. P. Hansen, E. G. J. Luttmer, and J. A. Scheinkman, 1997, Short-Term Interest Rates as Subordinated Diffusions, Review of Financial Studies 10, 525-577.

Corsi, Fulvio, 2009, A simple approximate long-memory model of realized volatility, Journal of Financial Econometrics 7, 174-196.

Corsi, Fulvio, Stefan Mittnik, Christian Pigorsch, and Uta Pigorsch, 2008, The Volatility of Realized Volatility, Econometric Reviews 27, 46-78.

Corsi, Fulvio, and Roberto Renò, 2012, Discrete-Time Volatility Forecasting With Persistent Leverage Effect and the Link With Continuous-Time Volatility Modeling, Journal of Business \& Economic Statistics 30, 368-380.

Danielsson, Jon, and Casper G De Vries, 2000, Value-at-Risk and Extreme Returns, Annales d'Economie et de Statistique 60, 239-270.

Danielsson, Jon, and Jean Pierre Zigrand, 2006, On time-scaling of risk and the squareroot-of-time rule, Journal of Banking and Finance 30, 2701-2713. 
Dennis, Patrick, and Stewart Mayhew, 2002, Risk-Neutral Skewness: Evidence from Stock Options, Journal of Financial and Quantitative Analysis 37, 471-493.

Dennis, Patrick, Stewart Mayhew, and Chris Stivers, 2006, Stock Returns, Implied Volatility Innovations, and the Asymmetric Volatility Phenomenon, Journal of Financial and Quantitative Analysis 41, 381.

Diebold, Francis X, Todd A Gunther, and Anthony S Tay, 1998, Evaluating Density Forecasts with Applications to Financial Risk Management, International Economic Review 39, 863-883.

Drost, C., and T. F. Nijman, 1993, Temporal Aggregation of Garch Processes, Econometrica $61,909-927$.

Embrechts, Paul, Roger Kaufmann, and Pierre Patie, 2005, Strategic Long-Term Financial Risks : Single Risk Factors, Computational Optimization and Applications 32, 61-90.

Engle, Robert F, and Giampiero M Gallo, 2006, A multiple indicators model for volatility using intra-daily data, Journal of Econometrics 131, 3-27.

Eraker, Bjørn, 2004, Do Stock Prices and Volatility Jump? Reconciling Evidence from Spot and Option Prices, Journal of Finance 59, 1367-1403.

Eraker, Bjòrn, Michael Johannes, and Nicholas Polson, 2003, The Impact of Jumps in Volatility and Returns, The Journal of Finance 58, 1269-1300.

Gilder, Dudley, Mark B. Shackleton, and Stephen J. Taylor, 2014, Cojumps in stock prices: Empirical evidence, Journal of Banking and Finance 40, 443-459.

Gonzalez-Rivera, Gloria, Tae-Hwy Lee, and Santosh Mishra, 2004, Forecasting volatility : A reality check based on option pricing, utility function, value-at-risk, and predictive likelihood, International Journal of Forecasting 20, 629-645.

Hansen, Peter R., and Asger Lunde, 2005a, A forecast comparison of volatility models: does anything beat a $\operatorname{GARCH}(1,1)$ ?, Journal of Applied Econometrics 20, 873-889.

Hansen, Peter Reinhard, and Asger Lunde, 2005b, A realized variance for the whole day based on intermittent high-frequency data, Journal of Financial Econometrics 3, $525-554$.

Hansen, Peter R., Asger Lunde, and James M. Nason, 2011, The Model Confidence Set, Econometrica 79, 453-497. 
Hautsch, Nicholaus, and Yangguoyi Ou, 2008, Stochastic Volatility Estimation Using Markov Chain Simulation, in Wolfgang Hardle, Nikolaus Hautsch, and Ludger Overbeck, eds.: Applied Quantitative Finance (Springer, ).

Heston, S.L., 1993, A Closed-Form Solution for Options With Stochastic Volatility with Applications to Bond and Currency Options, Review of Financial Studies 6, 327-343.

Hujer, Reinhard, and Sandra Vuletic, 2007, Econometric analysis of financial trade processes by discrete mixture duration models, Journal of Economic Dynamics and Control 31, 635-667.

Ignatieva, Katja, Paulo Rodrigues, and Norman Seeger, 2015, Empirical Analysis of Affine Versus Nonaffine Variance Specifications in Jump-Diffusion Models for Equity Indices, Journal of Business \& Economic Statistics 33, 68-75.

Jacquier, Eric, Nicholas Polson, and Peter Rossi, 1994, Bayesian Analysis of Stochastic Volatility Models, Journal of Business \& Economic Statistics 12, 371-417.

Jacquier, Eric, Nicholas Polson, and Peter Rossi, 2004, Bayesian Analysis of Stochastic Volatility Models with Fat-Tails and Correlated errors, Journal of Econometrics 122, 185-212.

Jiang, George J., and Tong Yao, 2013, Stock Price Jumps and Cross-Sectional Return Predictability, Journal of Financial and Quantitative Analysis 48, 1519-1544.

Johannes, Michael, and Nicholas Polson, 2006, MCMC Methods for Financial Econometrics, in Yacine Aït-Sahalia, and Lars Hansen, eds.: Handbook of Financial Econometrics (Elsevier, ).

Jones, Christopher S., 2003a, Nonlinear Mean Reversion in the Short-Term Interest Rate, Review of Financial Studies 16, 793-843.

Jones, Christopher S., 2003b, The dynamics of stochastic volatility: evidence from underlying and options markets, Journal of Econometrics 116, 181-224.

Kass, R.E., and A.E. Raftery, 1995, Bayes Factors, Journal of the American Statistical Association 90, 773-795.

Kuester, Keith, Stefan Mittnik, and Marc S. Paolella, 2006, Value-at-risk prediction: A comparison of alternative strategies, Journal of Financial Econometrics 4, 53-89.

Liu, Lily Y., Andrew J. Patton, and Kevin Sheppard, 2015, Does anything beat 5-minute RV? A comparison of realized measures across multiple asset classes, Journal of Econometrics 187, 293-311. 
Luciani, Matteo, and David Veredas, 2015, Estimating and forecasting large panels of volatilities with approximate dynamic factor models, Journal of Forecasting 34, 163176.

Maheu, John M., and Thomas H. McCurdy, 2004, News Arrival, Jump Dynamics, and Volatility Components for Individual Stock Returns, Journal of Finance 59, 755-793.

Maheu, John M., and Thomas H. McCurdy, 2011, Do high-frequency measures of volatility improve forecasts of return distributions?, Journal of Econometrics 160, 69-76.

McNeil, Alexander, Rudiger Frey, and Paul Embrechts, 2015, Quantitative Risk Management: concepts, techniques and tools. (Princeton University Press Oxford).

McNeil, Alexander J, and Rüdiger Frey, 2000, Estimation of tail-related risk measures for heteroscedastic financial time series: an extreme value approach, Journal of Empirical Finance 7, 271-300.

Mijatovic, A., and P. Schneider, 2014, Empirical Asset Pricing with Nonlinear Risk Premia, Journal of Financial Econometrics 12, 479-506.

Nieto, Rosa Maria, and Esther Ruiz, 2016, Frontiers in VaR forecasting and backtesting, International Journal of Forecasting 32, 475-501.

Pan, Jun, 2002, The Jump-Risk Premia Implicit in Options: Evidence from an Integrated Time-Series Study, Journal of Financial Economics 63, 3-50.

Patton, Andrew J., and Kevin Sheppard, 2015, Good Volatility, Bad Volatility: Signed Jumps and the persistence of volatility, Review of Economics and Statistics 96, 638-647.

Rossi, Eduardo, and Paolo Santucci de Magistris, 2014, Estimation of long memory in integrated variance, Econometric Reviews 33, 785-814.

Şerban, Mihaela, John Lehoczky, and Duane Seppi, 2008, Cross-Sectional Stock Option Pricing and Factor Models of Returns, Working Paper.

Shimotsu, Katsumi, and Peter C B Phillips, 2006, Local Whittle estimation of fractional integration and some of its variants, Journal of Econometrics 130, 209-233.

Taylor, Stephen J., 1982, Financial Returns Modelled by the Product of Two Stochastic Processes, a Study of Daily Sugar Prices 1961-1979, in O. D. Anderson, eds.: Time Series Analysis: Theory and Practice (North-Holland, ). 
Wang, Jying N., Jin Huei Yeh, and N. Y P Cheng, 2011, How accurate is the square-rootof-time rule in scaling tail risk: A global study, Journal of Banking and Finance 35, $1158-1169$.

Yu, Jun, 2005, On leverage in a stochastic volatility model, Journal of Econometrics 127, $165-178$. 


\section{Research Impact}

Financial modeling should not aim only to the development of new methods and models per se but rather should shed light on possible deficiencies in regulation and practice in financial markets. This chapter summarizes the main findings to the light of their possible applications for practitioners and regulators. This thesis sheds light on some issues related to systemic risk and risk management.

Chapter 2 reveals the characteristics of single stocks both in isolation from the index and in comparison to the it. This study has therefore important implications both for practitioners and policy makers as it highlights that systematic tail events happen in concordance with significant macroeconomic news. From a practitioner's perspective this implies that when these events happen the benefits arising from portfolio diversification are less pronounced as a large cross-section of companies exhibit an extreme negative return. From a regulator perspective, this chapter sheds light on which are the companies/sectors that exhibit extreme downward returns more frequently compared to the others therefore highlighting which are the riskiest companies.

Chapter 3 focuses on the usage of High-Frequency data for better forecasting returns. distribution. This chapter develops new models investigating which features of the data are relevant for daily risk measures. In a model validation exercise, this study concludes that High-Frequency data provide a simpler but valid alternative to existing model.

Chapter 4 takes the perspective of the risk manager of an institution that has a longer investment horizon and investigates how an annual risk measure should be estimated. Therefore, this study draws implications relevant for both practitioners and regulators. This study highlights that due to the empirical properties of volatility, measured using High-Frequency data, the annual return distribution highly differs from the normal one therefore implying a more conservative VaR estimate. Further, this study provides a simple rule of thumb to obtain the annual VaR when the daily one is at hand. 


\section{Biography}

Alessandro Pollastri was born on November 19th, 1988 in Florence, Italy. He earned a B.Sc. in Economics and Finance from the University of Siena in 2010. In 2013 he received a M.Sc. in Finance cum Laude from the University of Siena. In 2014 he received a M.Res in Econometrics with Distinction from the Université Libre de Bruxelles.

After graduating, Alessandro joined the Department of Finance at Maastricht University to pursue a doctorate degree under the supervision of Prof. dr. Peter Schotman and Dr. Stefan Straetmans. During his PhD he visited the University of Verona hosted by Prof. Dr. Roberto Renó.

The results of this effort are collected in this thesis. Alessandro has presented this research at several international conferences and seminars. He is currently a Post-Doctoral fellow at Scuola Normale Superiore in Pisa, Italy. 